\title{
Multiplexed cell-based assays to profile GPCR activities and cellular signalling
}

\author{
Dissertation \\ for the award of the degree \\ "Doctor rerum naturalium" \\ of the Georg-August-University Göttingen
}

\author{
submitted by \\ Sabrina Galinski \\ from Einbeck, Germany
}

Göttingen, 2016 
Thesis committee:

Prof. Dr. Moritz Rossner (Reviewer)

Molecular Neurobiology, Department of Psychiatry,

Ludwig-Maximilians-University Munich

Prof. Dr. Martin Göpfert (Reviewer)

Department of Cellular Neurobiology,

Georg-August-University Göttingen

Extended thesis committee:

Prof. Dr. Ernst Wimmer

Department of Developmental Biology,

Georg-August-University Göttingen

\section{Prof. Dr. Michael Sereda}

Molecular and Translational Neurology, Department of Neurogenetics, Max Planck Institute of Experimental Medicine, Göttingen

Prof. Dr. Ralf Heinrich

Department of Cellular Neurobiology,

Georg-August-University Göttingen

\section{Dr. Katrin Willig}

Nanoscale Microscopy and Molecular Physiology of the Brain, Max Planck Institute of Experimental Medicine, Göttingen

Date of Disputation: 25.02.2016 


\section{Declaration}

Herewith I declare that I prepared the PhD thesis entitled: 'Multiplexed cell-based assays to profile GPCR activities and cellular signalling' on my own and with no other sources and aids than quoted.

Sabrina Galinski

$18^{\text {th }}$ January, 2016

Göttingen 


\section{Contents}

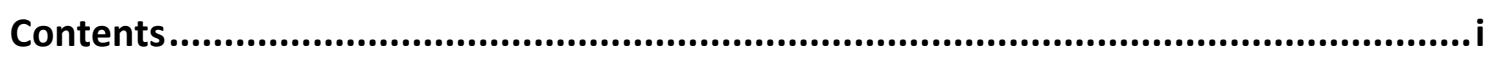

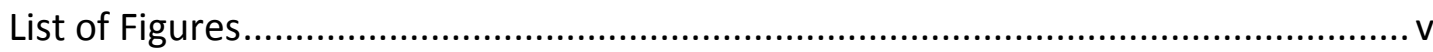

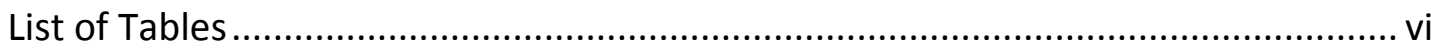

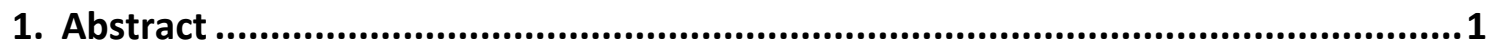

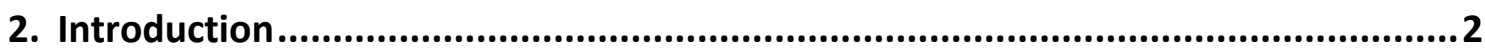

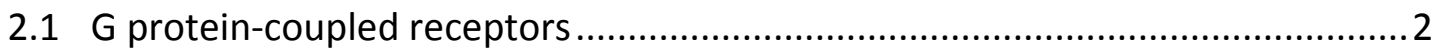

2.1.1 Activation of $\mathrm{G}$ protein coupled receptors...........................................2

2.1.2 Termination of $\mathrm{G}$ protein-coupled receptor activity................................3

2.1.3 G protein-coupled receptor signalling ................................................... 4

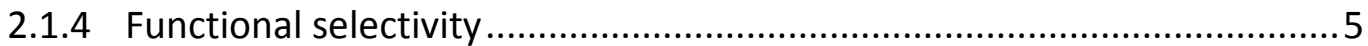

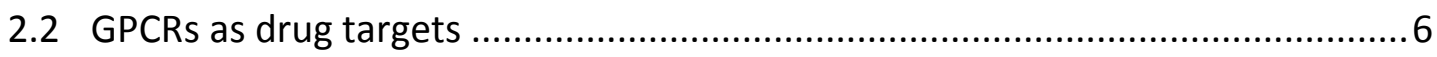

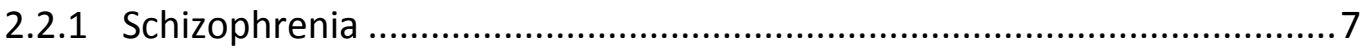

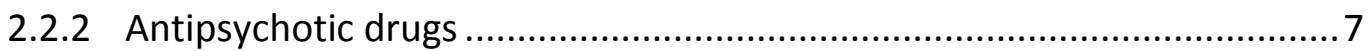

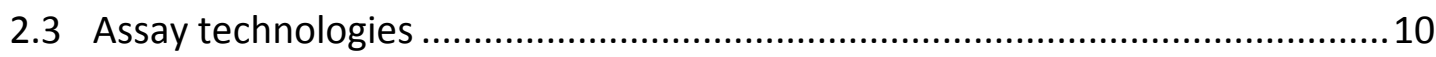

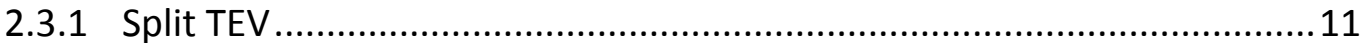

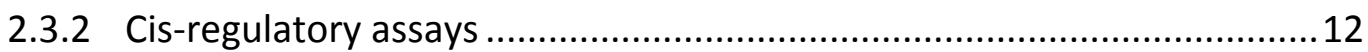

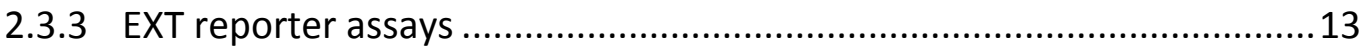

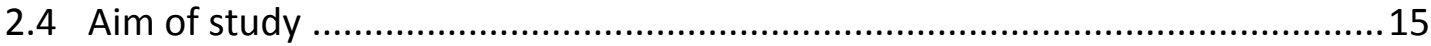

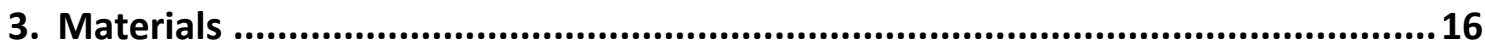

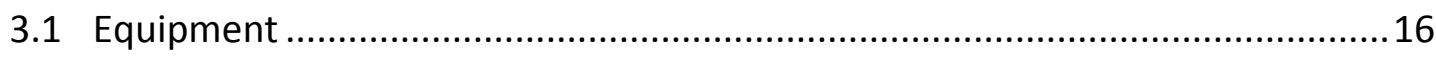

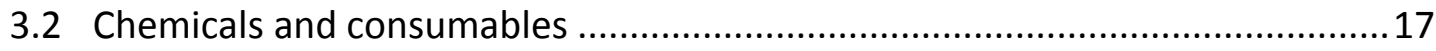

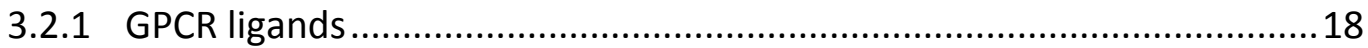

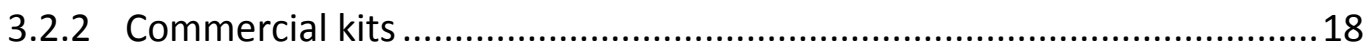

3.2.3 NGS Reagents \& Chips ................................................................. 18

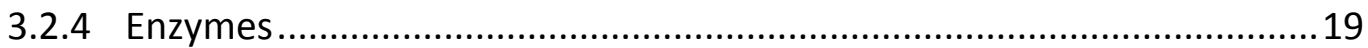

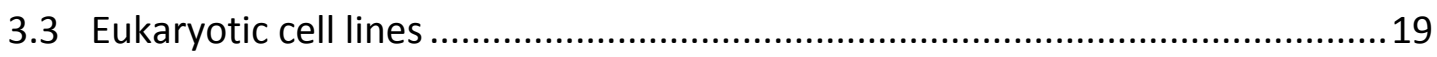

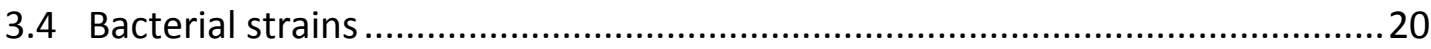

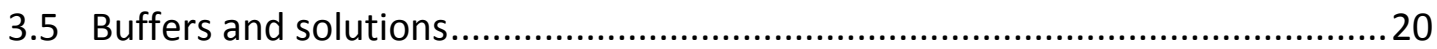

3.5.1 Molecular biology solutions .......................................................... 20 


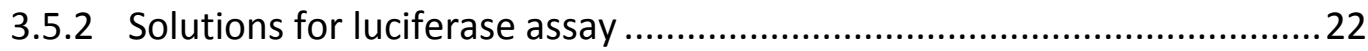

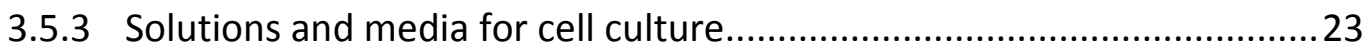

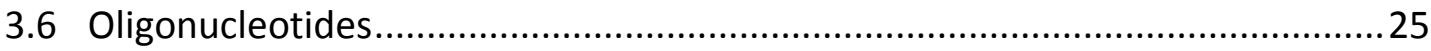

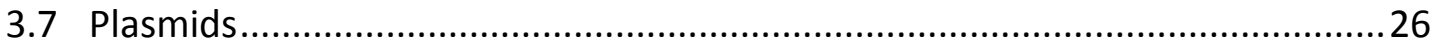

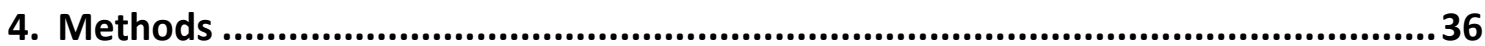

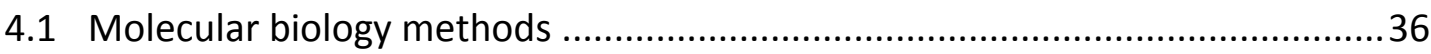

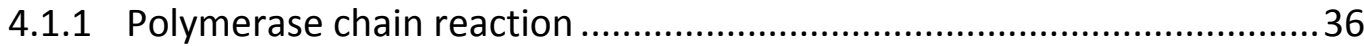

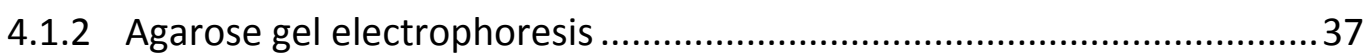

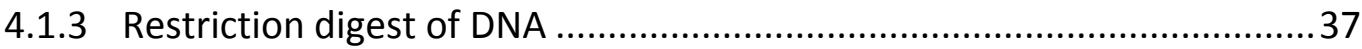

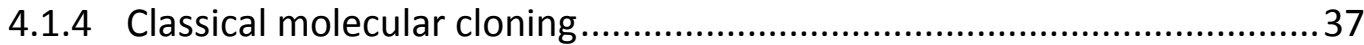

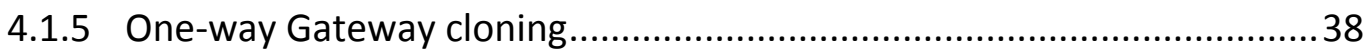

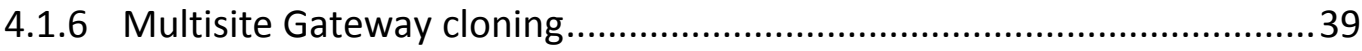

4.1.7 Photometric concentration determination of nuclear acids ..................41

4.1.8 Fluorometric concentration determination of nuclear acids..................42

4.1.9 Transformation of chemically competent bacteria............................... 42

4.1.10 Transformation of electro competent bacteria .....................................42

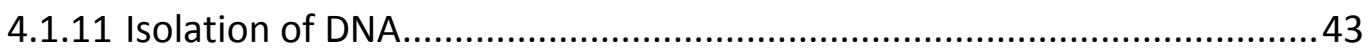

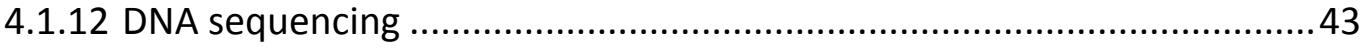

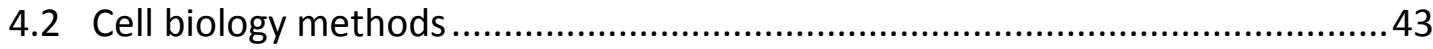

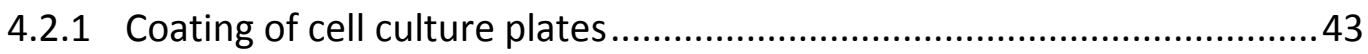

4.2.2 Culturing and passaging of eukaryotic cells .........................................43

4.2.3 Freezing of mammalian cells ................................................................4

4.2.4 Thawing of mammalian cells ............................................................... 44

4.2.5 Transfection of eukaryotic cells...........................................................45

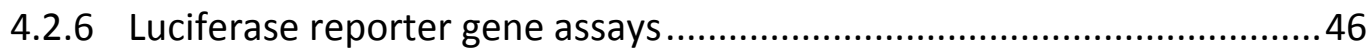

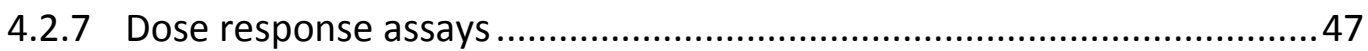

4.3 Preparation of EXT sequencing libraries ....................................................... 48

4.3.1 RNA isolation and purification............................................................ 48

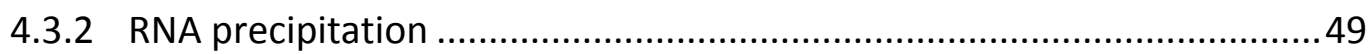

4.3.3 First strand cDNA synthesis .............................................................. 49

4.3.4 Composition of external calibrator mix.............................................50

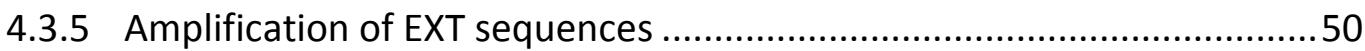


4.3.6 Attachment of Ion adapters and sample coding ...............................51

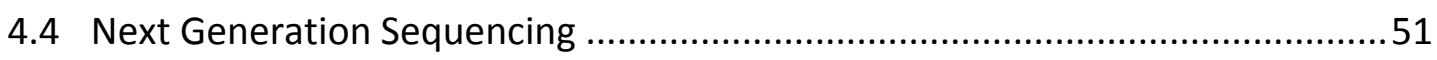

4.4.1 Preparation of sequencing templates ..................................................5 54

4.4.2 Sample sequencing on Ion Torrent sequencer .....................................5 54

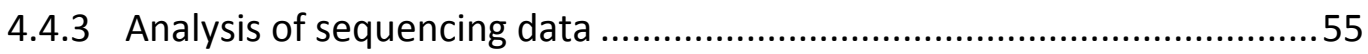

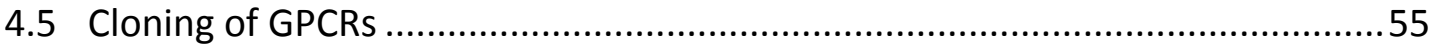

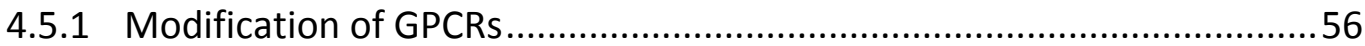

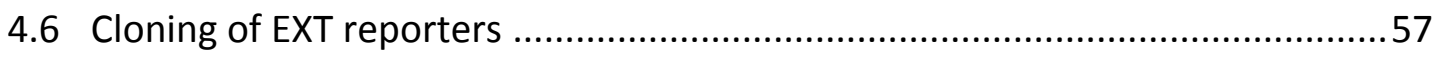

4.6.1 10xUAS-EXT reporters for GPCR activation assays.................................57

4.6.2 Sensor-EXT reporters for GPCR signalling assay..................................57

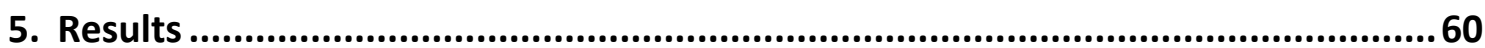

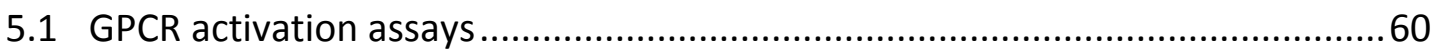

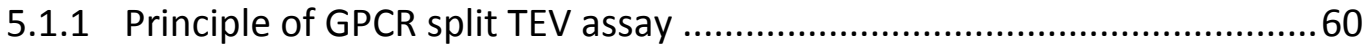

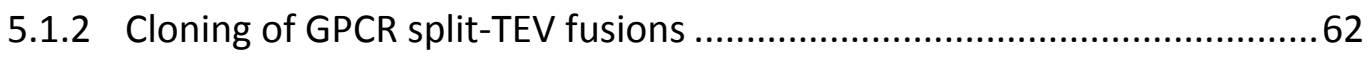

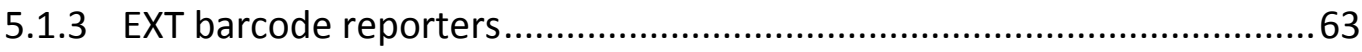

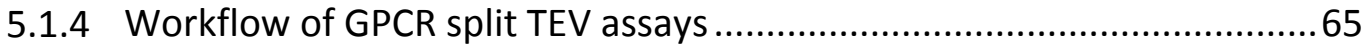

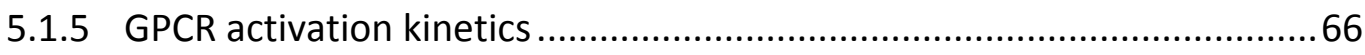

5.1.6 Luciferase-based evaluation of GPCR activation ...................................68

5.1.7 Dose-dependent GPCR activation and inhibition.................................. 73

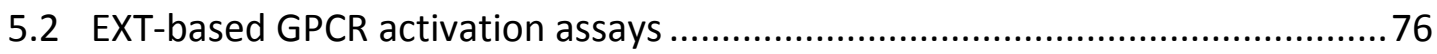

5.2.1 Principle of multiplexed GPCR activation EXT assays..............................76

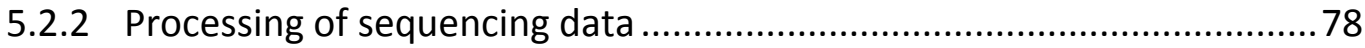

5.2.3 GPCR activation profiles in U2OS cells ...............................................79

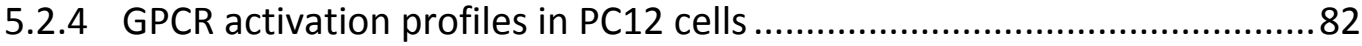

5.2.5 Profiles of antipsychotic drug effects on GPCR activation ..................... 83

5.3 EXT-based measurement of GPCR downstream signalling ..............................90

5.3.1 Principle of multiplexed GPCR signalling EXT assay ...............................90

5.3.2 Profiles of GPCR-mediated downstream signalling..............................91

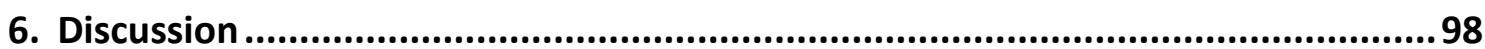

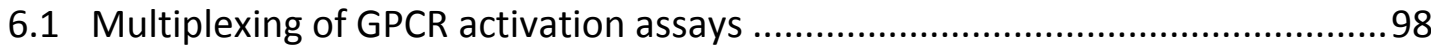

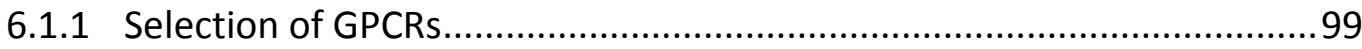


6.1.2 Time limitations of GPCR split TEV assays ........................................ 99

6.1.3 Split TEV assays as tool for monitoring GPCR activations .....................100

6.1.4 GPCR activity profiles monitored in multiplexed split TEV assays .........101

6.1.5 Profiling the action of drugs on receptor activation .............................102

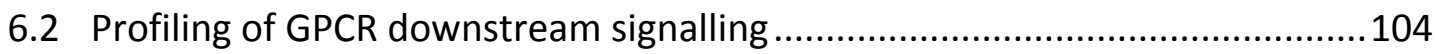

6.3 Concepts and future aspects of GPCR profiling assays .................................. 105

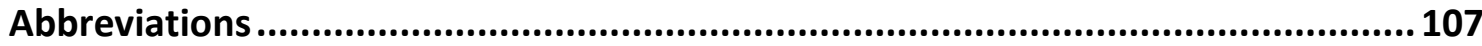

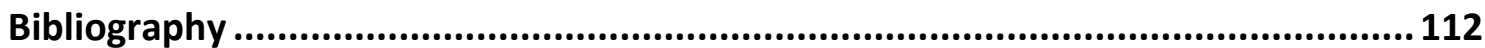

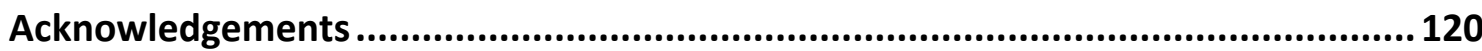




\section{List of Figures}

Figure 1: Schematic representation of functional selectivity (biased agonism). 5

Figure 2: Graphical representation of relative binding affinities of antipsychotic drugs to multiple receptors.

Figure 3: Graphical representation of the split TEV technique.

Figure 4: Structure of EXTs.

Figure 5: Scheme of the three fragments assembly using Multisite Gateway

Technology. 40

Figure 6: Workflow of EXT sequencing library preparation. 48

Figure 7: Workflow of Next Generation Sequencing by lon Torrent technology. 53

Figure 8: Modification of GPCR signal peptides. 56

Figure 9: GPCR split TEV constructs and principle of GPCR split TEV assays. 61

Figure 10: Structure of EXT barcode reporter constructs and library. 63

Figure 11: Control of reporter inducibility and potential substance interference. 64

Figure 12: Timeline and workflow of different types of GPCR split TEV assays. 66

Figure 13: Kinetic measurement of ligand dependent GPCR activation. .67

Figure 14: Split TEV luciferase assay to monitor ligand dependent GPCR activation in

U2OS cells.

Figure 15: Split TEV luciferase assay to monitor ligand dependent GPCR activation in PC12 cells.

Figure 16: Split TEV luciferase assay to monitor ligand dependent GPCR activation in $\mathrm{CHO}$ cells. .72

Figure 17: Dose-dependent GPCR activation by agonists in luciferase assays...............74

Figure 18: Dose-dependent GPCR inhibition by antagonists in luciferase assays..........75

Figure 19: Schematic design of multiplexed EXT-based GPCR activation assays. ..........77

Figure 20: Data processing workflow of GPCR activation EXT assays. .......................... 78

Figure 21: Normalisation of sequencing raw data to total reads................................ 79

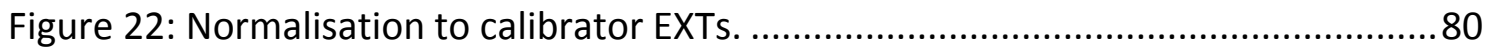

Figure 23: Profiles of GPCR activations in U2OS cells............................................... 81

Figure 24: Profiles of GPCR activations in PC12 tetOFF cells................................... 82

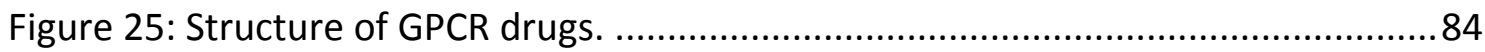


Figure 26: Profiles of drug effects on GPCR activation and inhibition

Figure 27: Analysis of drug effects on receptor activation in multiplexed GPCR EXT assay. 88

Figure 28: Luciferase-based validation of drug effects from GPCR EXT assay. 89

Figure 29: Schematic representation of EXT-based GPCR signalling assay. 90

Figure 30: GPCR signalling assay for monitoring GPCR and G protein-induced signalling.

Figure 31: Activity signals of G proteins on CRE reporter by EXT vs. luciferase measurement. 94

Figure 32: Signal profiles of G proteins and GPCRs using an EXT sensor library. .95

Figure 33: Clustering of GPCR induced signalling profiles and control conditions. 96

Figure 34: Clustering of G protein and GPCR induced signalling profiles 97

\section{List of Tables}

Table 1: List of plasmids. .26

Table 2: Overview of Ion Torrent sequencing devices and chips. ..............................52

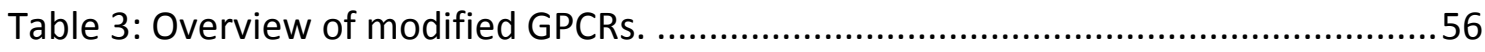

Table 4: List of cis-elements and endogenous promoters for sensor-EXT reporters.....58

Table 5: Overview of all GPCR split TEV fusion constructs used in GPCR activation assays. 62

Table 6: Summary table of GPCR performance in luciferase assays. 69

Table 7: Overview of GPCRs and corresponding compounds used in GPCR signalling assay. 


\section{Abstract}

G protein-coupled receptors (GPCRs) are the largest class of cell surface receptors that are implicated in the physiological regulation of many biological processes. They mediate signals through complex networks of $\mathrm{G}$ protein-dependent and independent signalling pathways. The high diversity of GPCRs and their physiological functions make them to primary targets for therapeutic drugs. The property of drugs to potentially modulate multiple targets, termed polypharmacology, is widely seen as undesired source for adverse side effects. However, polypharmacology may also explain the clinical efficacy of some drug classes, such as antipsychotic drugs used for the treatment of psychiatric diseases.

In this thesis, a GPCR profiling system is introduced comprising two aspects of multiplexed GPCR assays monitoring multiple selected events both at the level of receptor activation and downstream cellular signalling. Firstly, the multiplexed GPCR activity assay combines split TEV and EXTassay technologies and enables simultaneous measurements of receptor activities for multiple GPCR-ligand combinations within one experiment. In proof-of-principle experiments, the specificity of endogenous agonists as well as the polypharmacological effects of described antipsychotics on GPCR activities was demonstrated. Secondly, the multiplexed GPCR signalling assay allows monitoring multiple cellular downstream signalling events following to GPCR activation. Both profiling approaches use molecular barcodes as reporters that are invariably linked to either a single GPCR activity or cellular signalling event, thus enabling a precise and simultaneous measurement of individual events in a global profiling setup. The assay was successfully applied to different GPCRs, their correlation to $G$ protein-coupled signalling and downstream signalling activities.

In summary, the multiplexed assays presented constitute a flexible and scalable approach, which enables simultaneous profiling of receptor activities and downstream signalling, and offer a thorough analysis of compound actions in living cells. 


\section{Introduction}

\subsection{G protein-coupled receptors}

Extracellular signals are transduced into cellular responses by signal transduction pathways. A central factor of these signal transduction pathways are membranebound receptors, which act as sensors on the cell surface and integrate extracellular signals by activating intracellular effector proteins. These effector proteins induce distinct intracellular downstream signalling cascades. The largest and most investigated class of membrane-bound receptors are the $\mathrm{G}$ protein-coupled receptors (GPCRs), with more than 800 members in the human genome (Lagerstrom and Schioth, 2008). All members of this class are composed of seven transmembrane $\alpha$-helices connected by three intra- and three extracellular loops of varying length, an extracellular $\mathrm{N}$-terminus and an intracellular C-terminus (Palczewski et al., 2000). GPCRs are activated by a variety of extracellular ligands like small molecules, peptides, hormones, neurotransmitters, odorants, or even light. Due to this diversity GPCRs are implicated in the regulation of a large variety of biological processes, such as neuronal activity, immune response, cell growth and differentiation.

\subsubsection{Activation of $\mathrm{G}$ protein coupled receptors}

All GPCRs have the common feature of binding and activating heterotrimeric $G$ proteins. Binding of an extracellular ligand to an inactive GPCR induces a conformational change of the receptor. This interaction leads subsequently to an exchange of bound GDP (Guanosine diphosphate) by GTP (Guanosine triphosphate) at the $\mathrm{G} \alpha$ subunit of the coupled heterotrimeric G protein. This change from GDP to GTP triggers the dissociation of the $G \alpha$ subunit from the $G \beta \gamma$ dimeric subunit and the concomitant release of both subunits from the receptor. The free receptor can then rebind and activate another $G$ protein until the receptor is deactivated. The two dissociated subunits, the $G \alpha$ and the $G \beta \gamma$ dimers, now interact and modulate downstream effectors, which in turn regulate intracellular concentrations of second messengers. This in turn, results in a physiological response, usually caused by the 
downstream regulation of gene expression (Hepler and Gilman, 1992; Wettschureck and Offermanns, 2005).

\subsubsection{Termination of $\mathrm{G}$ protein-coupled receptor activity}

There are two mechanisms to deactivate GPCR signalling. The first mechanism is the short-term deactivation of G proteins by hydrolysis of GTP (active state) to GDP (inactive state) of the $\mathrm{Ga}$ subunit. The hydrolysis is catalysed on the one hand by the intrinsic GTPase activity of the $\mathrm{G} \alpha$ subunit and on the other hand by regulators of $G$ protein signalling (RGS), which are also known as GTPase activating proteins (GAPs) (Xie and Palmer, 2007).

The second long-term mechanism is the desensitization of the active receptor. The conformation change as a result of agonist binding leads to the recruitment of $G$ protein coupled receptor kinases (GRKs). These kinases phosphorylate residues on the third intracellular loop and the $\mathrm{C}$ terminus of the receptor. The phosphorylation in turn promotes the binding of proteins from the $\beta$-arrestin family (Kohout and Lefkowitz, 2003). The binding of $\beta$-arrestin sterically hinders $G$ protein coupling and triggers the internalization of the GPCR. The internalization is promoted by the interaction of $\beta$-arrestin, the adaptors of the endocytic machinery, such as the AP2 adaptor complex, and clathrin to form clathrin-coated endocytotic vesicles. The endocytosis results either in the degradation of the receptor in lysosomes or in the receptor dephosphorylation and recycling back to the plasma membrane (Zhang et al., 1996). Regarding to the receptor- $\beta$-arresting complex and their trafficking patterns GPCRs can be classified as follows: 'class $A^{\prime}$ receptors (e.g. ADRB2, DRD1) show a strong affinity to $\beta$-arrestin 2 that is bound to the receptor at the cell surface but dissociates when the receptor internalizes (Oakley et al., 2000; Shenoy and Lefkowitz, 2003). This allows the dephosphorylation of the receptor and the trafficking back to the membrane (Pitcher et al., 1995). 'Class B' receptors (e.g. AVPR2) bind $\beta$-arrestin 1 and $\beta$-arrestin 2 with equal tight affinity and do not dissociate from each other but traffic together into endosomes (Oakley et al., 2000; Shenoy and Lefkowitz, 2003). 


\subsubsection{G protein-coupled receptor signalling}

The primary effector proteins and signal transducers of active GPCRs are the coupled heterotrimeric $\mathrm{G}$ proteins. The versatility of $\mathrm{G}$ protein-mediated signalling is based on different subtypes of $\mathrm{G}$ proteins that are named according to the $\mathrm{G} \alpha$ subunit. There are four main families of $G$ proteins, $G_{s}, G_{i}, G_{q}$ and $G_{12}$.

$G \alpha_{s}$ interacts and activates adenylate cyclase (AC), which converts ATP to cAMP (cyclic adenosine monophosphate) resulting in increased intracellular cAMP levels. cAMP acts as a second messenger and activates the enzyme protein kinase A (PKA), which in turn regulates the activity of other proteins by their phosphorylation, such as enzymes and transcription factors like the cAMP response element-binding protein (CREB).

In the $G_{i}$ pathway the $G \alpha_{i}$ subunit inhibits adenylate cyclase and therefore, the intracellular cAMP concentration decreases. Apart from the $\mathrm{G} \alpha$ subunit, also the $\mathrm{G} \beta \gamma$ complex induces signal transduction pathways. $G \beta \gamma$ regulated effectors are phosphoinositide 3-kinase (PI3K), potassium channels and phospholipase $\mathrm{C}$ beta (PLC- $\beta$ ) (Neves et al., 2002).

$G \alpha_{q}$ signals through the activation of PLC- $\beta$ that cleaves the membrane-bound phospholipid phosphatidylinositol 4,5-bisphosphate $\left(\mathrm{PIP}_{2}\right)$ into the two second messengers inositol trisphosphate $\left(\mathrm{IP}_{3}\right)$ and diacylglycerol (DAG). $I \mathrm{P}_{3}$ mobilises calcium release from intracellular stores to increase the cytosolic $\mathrm{Ca}^{2+}$ concentration, and activates further signalling events like the activation of nuclear factor of activated T-cells (NFAT). DAG interacts with and activates protein kinase C (PKC) that regulates other proteins (Neves et al., 2002).

$\mathrm{G}_{12}$ proteins activate the monomeric GTPase RhoA. RhoA in turn activates effectors such as Rho kinase (ROCK). Therefore, $\mathrm{G}_{12}$ proteins are involved in the regulation of the actin cytoskeleton, cell growth and cell migration as well as in gene transcription (Siehler, 2007).

The second important signal transducers of GPCRs are $\beta$-arrestins. Besides their function of GPCR desensitisation, they are also capable to act as adaptor proteins and initiate distinct signal transduction pathways. In the late 1990s, the recruitment of c-Src to $\beta$-arrestin 1 and the activation of the mitogen-activated protein (MAP) kinases extracellular signal-regulated kinases (Erk1 and Erk2) was discovered (Luttrell et al., 1999). Later, multiple interacting partners of $\beta$-arrestin 1 and $\beta$-arrestin 2 were found, 
such as phosphoinositide 3-kinase (PI3K) and nuclear factor-KB (NFKB) (DeWire et al., 2007; Lefkowitz and Shenoy, 2005). The discovery of $\beta$-arrestin mediated signalling leads to the changing of the classical model of linear $\mathrm{G}$ protein signalling upon receptor activation to a complex signalling network of $G$ protein and $\beta$-arrestin-mediated pathways.

\subsubsection{Functional selectivity}

In pharmacology, ligands can be described by two properties, affinity and efficacy. Affinity is the ability of a ligand to bind to a certain receptor and efficacy is the ability of the ligand-receptor complex to produce a cellular response (Rajagopal, 2013; Stephenson, 1956). In the classical or traditional concept of pharmacology, ligands act either as agonists, antagonists or inverse agonists through a specific receptor. Each of these properties is consistent with the induced cellular response pathways coupled to that receptor. In this concept an agonist activates all signalling pathways linked to that receptor in the same way as the corresponding endogenous ligand, whereas an antagonist blocks all signalling pathways to the same degree. A new concept termed functional selectivity or biased agonism postulates that a ligand can induce differential signalling pathways downstream of a given receptor. Thus, a ligand may act as an agonist on some signalling pathways coupled to the receptor and simultaneously as an antagonist on other pathways (Figure 1).

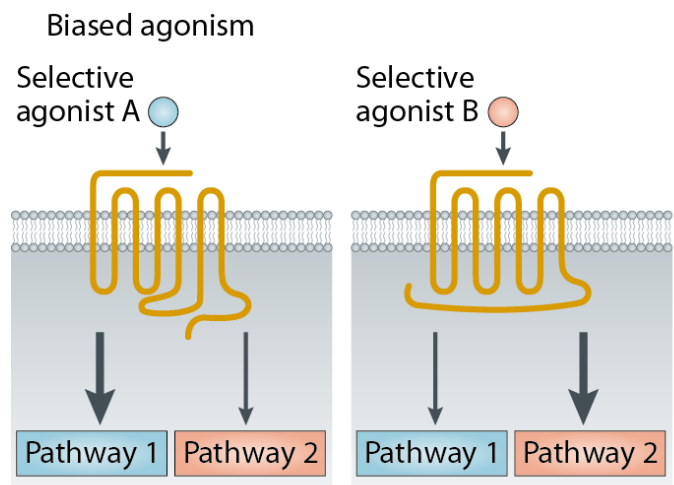

Figure 1: Schematic representation of functional selectivity (biased agonism).

Agonist $A$ is functional selective in activating pathway 1 whereas agonist $B$ induces pathway 2 . Adapted from Kenakin and Christopoulos, 2013. 
The degree to which given signalling pathways are activated is dependent on the ligand bound to the receptor. Against the classical concept where ligand efficacy is cell type-independent and therefore can be used to predict the activity in any systems, functional selective ligands can have multiple efficacies, which are dependent on the cell system and the receptor coupled effectors (Chang and Bruchas, 2014; Kenakin, 2011; Kenakin and Christopoulos, 2013; Urban et al., 2007).

The developing concept of functional selectivity is based on reports of serotonin receptor (Berg et al., 1998) and dopamine receptor (Mottola et al., 2002) agonists. It was observed that each ligand is selective for certain signalling pathways compared to the other ligands. Regarding GPCRs and the discovery of $\beta$-arrestin mediated signalling, ligands can be functionally selective either towards $G$ protein- or $\beta$-arrestin-mediated signalling (Chang and Bruchas, 2014; Rajagopal et al., 2010; Whalen et al., 2011).

This functional selectivity of ligands offers new opportunities for therapeutic drug development. Ligands can be identified or developed that are able to activate beneficial signalling pathways and prevent other pathways that are responsive for unwanted effects (e.g. side effects or toxicity).

\subsection{GPCRs as drug targets}

Due to the complexity of GPCRs and their signal transduction mechanisms the deregulation of GPCRs and their mediated signalling pathways is implicated in the pathophysiology of various diseases. They play a role in metabolic disorders, such as obesity and type 2 diabetes, in immune diseases and in neurodegenerative diseases like Alzheimer's and Parkinson's disease (Heng et al., 2013). Furthermore, GPCRs are involved in the pathophysiology and the treatment of major psychiatric disorders like schizophrenia and bipolar disorder (Catapano and Manji, 2007; Heng et al., 2013). Their roles in the pathophysiology of this variety of diseases make GPCRs to key targets for pharmacological research. Currently, GPCRs are targeted by approximately $30-40 \%$ of all marketed drugs (Stevens et al., 2013). One important class of GPCR targeting medications are antipsychotic drugs for the treatment of psychiatric diseases like schizophrenia (Komatsu, 2015). 


\subsubsection{Schizophrenia}

Schizophrenia is a severe mental disorder characterized by perception of reality and disturbed social behaviour. The lifetime prevalence of schizophrenia ranges between $0,3 \%$ and $0,7 \%$ with an onset in the late adolescence and early adulthood (McGrath et al., 2008). The symptoms are divided into the terms of positive and negative symptoms and cognitive deficits. The positive symptoms, which include e.g. hallucinations, delusions and disorganized thinking and speech, can be effectively ameliorated by the treatment with antipsychotic drugs. Whereas the negative symptoms including social and emotional withdraw, lack of motivation and poverty of speech as well as cognitive deficits can only slightly or not effective be treated (Conn et al., 2008; Miyamoto et al., 2012; Tandon et al., 2009).

The antipsychotic efficacy of antipsychotic drugs and the pro-psychotic effects of amphetamine led to the conceptualization of a role for dopamine in schizophrenia (Davis et al., 1991). The dopamine hypothesis involves a dysregulation of dopaminergic activities in the brain with an excess of activity in the mesolimbic dopamine pathway and an abnormal low activity in the mesocortical pathway (Davis et al., 1991). Nonetheless, the pathophysiology and aetiology of schizophrenia are still unknown (Insel, 2010).

\subsubsection{Antipsychotic drugs}

Antipsychotic drugs, also known as neuroleptics, are a class of psychotic medications with sedating and antipsychotic effects. They are mainly used in the treatment of schizophrenia and bipolar disorder. The main effect of antipsychotics is the reduction of positive symptoms, such as hallucinations and delusions.

The discovery of antipsychotic drugs is based on observations in the late $19^{\text {th }}$ century when it was found that the aniline dye methylene blue, a phenothiazine derivative, has antimalarial effects. During the early $20^{\text {th }}$ century, phenothiazine derivatives were found to have antihistaminic and sedative properties. In 1950, the phenothiazine derivative chlorpromazine was synthesised for use in clinical anaesthesia. Shortly thereafter, antipsychotic effects of chlorpromazine were discovered, and 
subsequently, chlorpromazine was introduced into psychiatric practice as the first antipsychotic drug. Later on, many antipsychotic drugs, such as haloperidol with similar chemical structures and mode of action, were developed and marketed. They are referred to as first-generation or typical antipsychotics. However, already shortly after the introduction of chlorpromazine the first side effects were recognized. The observed side effects are summarized as extrapyramidal symptoms (EPS) including Parkinson-like symptoms, akathisia, and dystonia. In 1961, the prevalence of EPS was estimated to be about 40\% (Ayd, 1961). In the late 1960s, a new antipsychotic drug came into the focus of pharmacologists and clinicians, the tricyclic dibenzodiazepine derivative clozapine. Clozapine turned out to be an effective antipsychotic inducing no or highly reduced EPS. The discovery of clozapine as an effective antipsychotic drug, atypical with regard to the lack of EPS side effects, lead to the development of a new class of antipsychotics, such as risperidone, olanzapine or quetiapine, then referred to as second-generation or atypical antipsychotics (Gründer et al., 2009). However, also atypical antipsychotics are associated with serious side effects, such as weight gain and metabolic side effects like diabetes and hyperlipidemia (Ücok and Gaebel, 2008).

Since their introduction atypical antipsychotics seemed promising regarding enhanced effectiveness and tolerability over the use of the older typical antipsychotics. However, this assumption was dampened in the last years by comparative studies like the clinical antipsychotic trials of intervention effectiveness (CATIE) study. It could not be shown unequivocally that second-generation antipsychotics are superior in their effectiveness than first-generation antipsychotics (Leucht et al., 2009; Lieberman et al., 2005). Although all current antipsychotics are effective in the reduction of positive symptoms they failed in the treatment of cognitive symptoms and have only little effect on the negative symptoms (Conn et al., 2008; Leucht et al., 2009). It is assumed that approximately $30 \%$ of patients are treatment resistant (Lally and MacCabe, 2015). The main mechanism of action of all currently used antipsychotic drugs is the reduction of dopaminergic activities in the brain by acting as full or partial antagonists on the dopamine D2 receptor (DRD2) and for most of the atypical antipsychotics the blocking of serotonin receptor activities, especially the serotonin receptor 2A (HTR2A) (Miyamoto et al., 2012). But the antagonism on these receptors alone cannot explain the antipsychotic efficacy. By analysing binding affinities of antipsychotics it could be 
shown that these drugs are pharmacologically complex by having pleiotropic effects. Figure 2 represents the relative affinity values $\left(\mathrm{K}_{\mathrm{i}}\right)$ of commonly used antipsychotics to a range of different receptors. It shows that all antipsychotics possess considerable affinities to a large number of aminergic GPCRs (Roth et al., 2004). However, the exact mode of action of antipsychotics especially with regard to the differences between typical and atypical and the induced side effects is still unclear (Miyamoto et al., 2012; Roth et al., 2004). But the affinity to multiple targets leads to the assumption that the clinical efficacy of these drugs is based on the complex polypharmacology. This evidence for polypharmacology promotes the idea of "magic shotguns" - drugs that exhibit their action through multiple targets and therefore can be better candidates in drug research for more complex diseases such as schizophrenia (Allen and Roth, 2011; Roth et al., 2004).

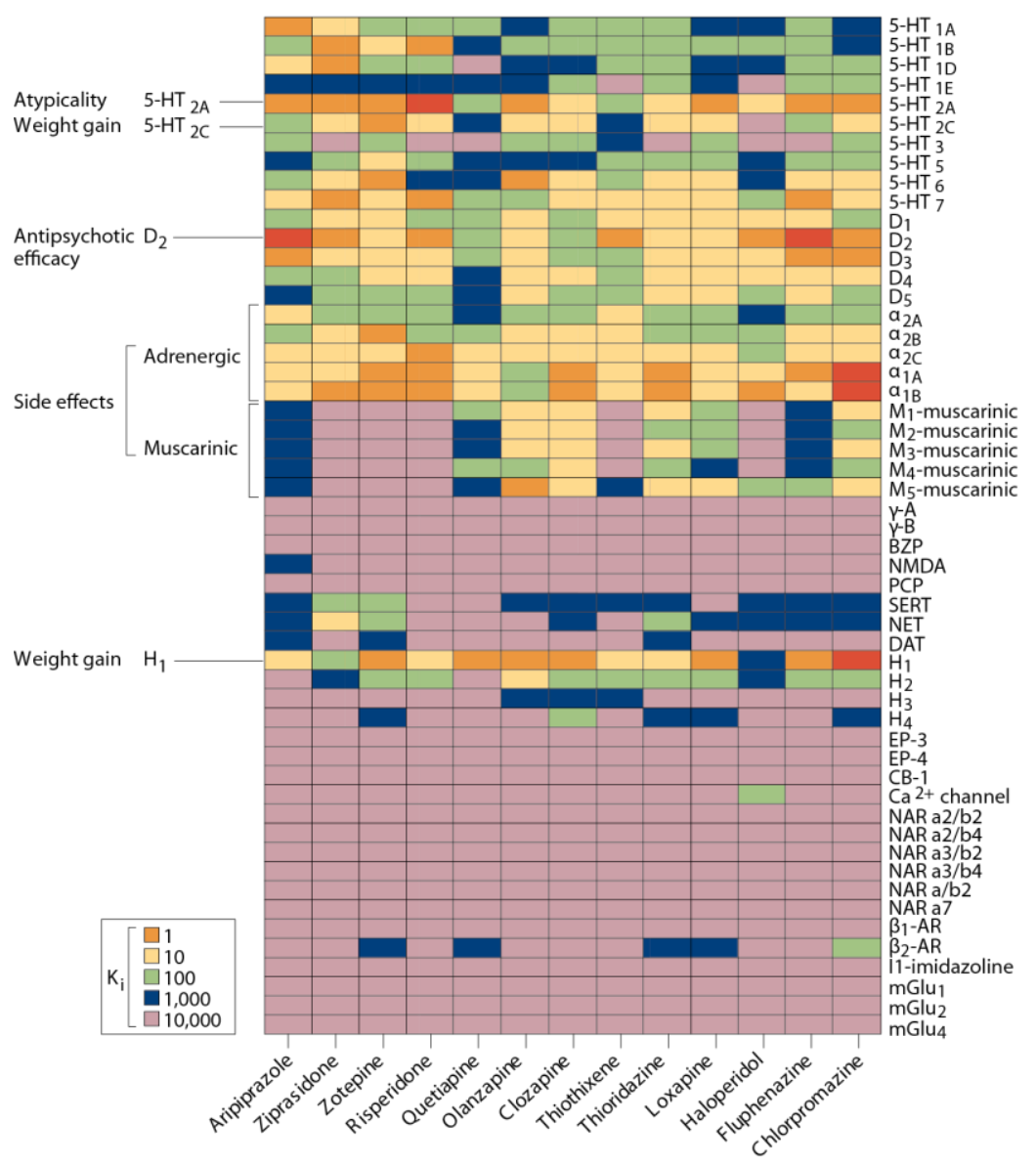

Figure 2: Graphical representation of relative binding affinities of antipsychotic drugs to multiple receptors.

The binding affinity values $\left(\mathrm{K}_{\mathrm{i}}\right)$ are from the PDSP $\mathrm{K}_{\mathrm{i}}$ database as part of the National Institute of Mental Health Psychoactive Drug Screening Program (https://pdspdb.unc.edu/pdspWeb/). All antipsychotics show a polypharmacology by complex binding affinities to multiple receptors. Adapted from Roth et al., 2004. 


\subsection{Assay technologies}

For the analysis of GPCRs and their mechanisms a high number of assay tools were developed over the last decades addressing the several steps of the GPCR signalling cascade. The properties of ligands to the particular receptors such as affinity or association/dissociation rates can be determined in ligand-binding assays using radiolabelled ligands or in newer assay formats with fluorescent-labelled ligands based on time-resolved fluorescence resonance energy transfer (TR-FRET) technology (Oueslati et al., 2015; Zhang and Xie, 2012). Direct G protein binding can be measured via GTP $\gamma S$ binding assays using the non-hydrolysable $\left[{ }^{35} \mathrm{~S}\right]-\mathrm{GTP} \gamma \mathrm{S}$ analogue (Harrison and Traynor, 2003). G protein signalling can be determined either by assays that measure changes in the concentration of the intracellular second messengers CAMP, $\mathrm{Ca}^{2+}$ and $\mathrm{IP}_{3}$ (Thomsen et al., 2005; Zhang and Xie, 2012) or by reporter gene assays using response elements, such as the cAMP responsive element (CRE) or the nuclear factor of activated T-cells responsive element (NFAT-RE) coupled to the expression of reporter proteins like luciferase, $\beta$-lactamase, or GFP (green fluorescence protein) (Cheng et al., 2010). Label-free whole cell assays were developed to measure the accumulation of cellular responses upon GPCR activation using optical or electrical, impedance-based biosensors (Miyano et al., 2014; Zhang and Xie, 2012). Approaches to assess the stimulation-dependent GPCR activation widely uses the interaction of $\beta$-arrestins and activated GPCR by several methods. GFP-tagged $\beta$-arrestin translocation upon receptor stimulation is tracked by fluorescence imaging (Oakley et al., 2002). Direct protein interactions assays based on Bioluminescence Resonance Energy Transfer (BRET) (Bertrand et al., 2002), Fluorescence Resonance Energy Transfer (FRET) (Eidne et al., 2002) or the enzyme complementation of $\beta$-galactosidase (Yan et al., 2002) or luciferase (Hattori and Ozawa, 2015) fragments. The Tango assay from Invitrogen is based on $\beta$-arrestin induced proximity of the TEV (tobacco etch virus) protease to its cleavage site resulting in transcription factor release and activation of a reporter gene (Barnea et al., 2008). An additional approach to monitor receptor-protein interactions are split TEV complementation assays (Djannatian et al., 2011; Wehr et al., 2006). 


\subsubsection{Split TEV}

Split TEV assays are a method to monitor protein-protein interactions in living cells. It is based on the fragment complementation of the Nla protease from the tobacco etch virus (TEV protease). The TEV protease is cleaved into two inactive fragments, an $\mathrm{N}$-terminal (NTEV) and a C-terminal fragment (CTEV) and both fragments are fused to the interacting proteins of interest. If an interaction of the proteins occurs both TEV fragments come into close proximity and reconstitute the functional protease activity. The cleavage site of the TEV protease is the specific amino acid sequence ENLYFQ'G (tevSite or tevS) that is fused to the NTEV fragment along with the artificial transcription factor Gal4-VP16 (GV) (Wehr et al., 2006). The Gal4-VP16 transcription factor is composed of the DNA binding domain of yeast Gal4 and the activating domain of the herpes simplex VP16 transcription factor (Sadowski et al., 1988). Upon interaction of proteins, the reconstituted TEV protease cleaves at tevS to release GV that translocates into the nucleus, where it activates a reporter gene of choice by binding to clustered Gal4-responsive elements (UAS, upstream activating sequence) (Figure 3). Reporter genes can be i.e. a fluorescent protein like the green fluorescence protein (GFP) or an enzyme like the firefly luciferase.

(1)

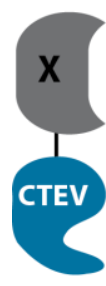

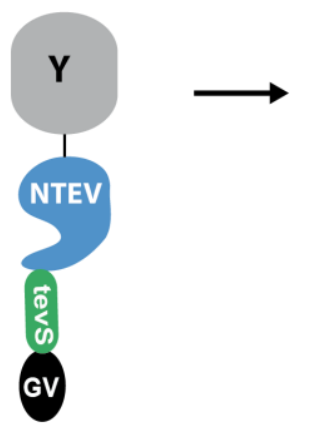

(2)

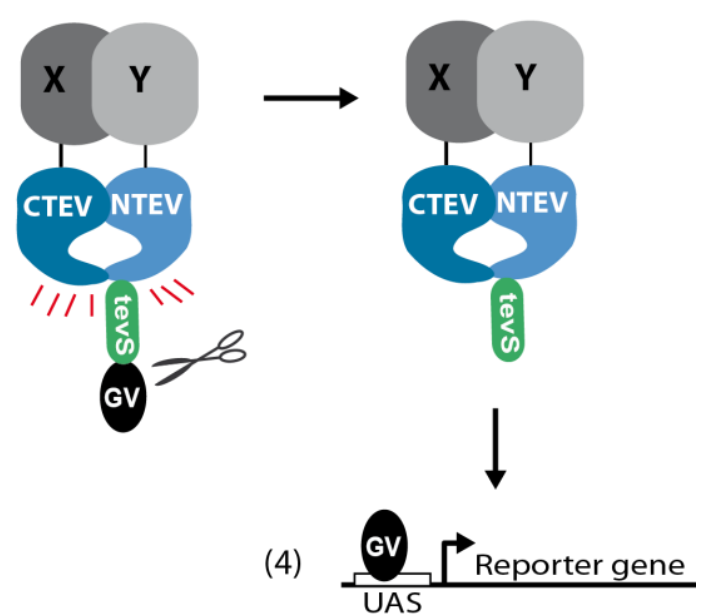

Figure 3: Graphical representation of the split TEV technique.

Split TEV assays are designed for monitoring protein-protein interactions. (1) The two protein interaction partners are fused to the $\mathrm{N}$-terminal and C-terminal fragments of the tobacco etch virus protease (TEV). (2) Upon protein-protein interaction both TEV fragments come into close proximity and reconstitute the functional protease. (3) The active protease leads to the cleavage of a TEV specific cleavage site (tevS) and thereby to the release of the artificial transcription factor Gal4-VP16 (GV), both linked to the NTEV fragment. (4) GV then activates a reporter gene by binding a GV responsive upstream activating sequence (UAS). 
The assay is sensitive to monitor protein-protein interactions in the cytosol and at the membrane. It was shown to be a robust assay by transient transfections for monitoring ERBB receptors as well as $G$ protein coupled receptors (Djannatian et al., 2011; Wehr and Rossner, 2015; Wehr et al., 2006, 2015). Furthermore, the split TEV technique could be successfully combined with EXT reporter assays for the simultaneous analysis of ERBB receptor tyrosine kinase dimerization (Botvinnik et al., 2010).

\subsubsection{Cis-regulatory assays}

Signal transduction cascades commonly result in the change of gene expression. This occurs by activation of transcription factors. Transcription factors are DNA binding proteins that recognize and bind specific DNA sequences, so called response or ciselements, within the regulatory region of target genes. Through the binding transcription factors regulate gene expression by either activation or repression of transcription of the adjacent gene. The activity of transcription factors can be monitored in reporter gene assays using reporter gene constructs. A reporter gene construct is composed of a regulatory DNA sequence, which can be artificially clustered cis-elements or an endogenous promoter sequence, coupled to a reporter gene encoding an easily detectable and quantifiable protein. Classical reporter proteins that are widely used are fluorescence proteins such as green fluorescent protein (GFP) or enzymes, such as firefly luciferase or $\beta$-galactosidase.

Reporter gene constructs (cis-reporters) carrying cis-elements indicative for defined transcription factors can be used as surrogate markers for distinct upstream signalling pathways. In GPCR research, classical cis-reporter constructs are the cAMP responsive element (CRE) for monitoring $G_{s}$ induced cAMP pathway or the nuclear factor of activated T-cells responsive element (NFAT-RE) for $\mathrm{G}_{\mathrm{q}}$ induced $\mathrm{Ca}^{2+}$ signalling (Cheng et al., 2010). However, cellular signalling in particular GPCR mediated signalling involves the induction of several transduction pathways and therefore the activation of multiple transcription factors. A method to assess the activities of multiple transcription factors in parallel is the application of a reporter construct library encoding several cis-elements coupled to reporters that can be detected simultaneously. Moreover, nucleic acid sequences can serve as reporters that are 
transcribed but not translated. Easily applicable detection and quantification methods for such cis-reporter libraries are for example capillary electrophoresis microarray hybridization or next generation sequencing (Botvinnik et al., 2010; Romanov et al., 2008).

\subsubsection{EXT reporter assays}

EXT reporter assays (EXTassays) represent a novel method for the simultaneous measurement of multiple cellular activities in living cells (Botvinnik et al., 2010). Here, unique expressed oligonucleotide tags (EXTs) serve as quantitative molecular barcode reporters that can be each invariably linked to different reporter gene assays, like the split TEV system or cis-regulatory reporter gene assays. EXTs are designed to replace classical reporter proteins and their limitations and enable the parallel assessment of numerous cellular events. Multiple EXT reporters can be used in one assay and be isolated and analysed as a pool by either microarray hybridisation or next generation sequencing (Botvinnik et al., 2010). Thereby, different cellular events can be monitored simultaneously within a reporter system or by combination of different reporter gene assays.

Each EXT is composed of a 49 base variable region, flanked by $5^{\prime}$ and $3^{\prime}$ non-variable regions. The variable region is arranged symmetrically by a core region of nine bases and ten "words" (W) (Figure 4). A "word" is a 4-nucleotide unit each consisting of three adenosines $(A)$ or thymidines $(T)$ and one cytosine $(C)$. Eight different "words" were used for EXT synthesis. The core region contains 9 bases of alternating $A, T(W)$ or $G$, $C(S)$ residues with $3 \mathrm{G}$ and $\mathrm{C}$ residues in the centre (Figure 4$)$. The complexity of a EXTlibrary is calculated as $8^{10}$ (10 positions with 8 words) times $2^{9}$ ( 2 bases at 9 core region positions) yielding in $5.5 \times 10^{11}$ different sequences with a nearly consistent melting temperature (Botvinnik and Rossner, 2012).

EXTassays were applied to monitor neuregulin-1-induced ErbB receptor tyrosine kinase dimerisations, and it could be shown that EXTs represent an improved measurement method regarding kinetic and sensitivity compared to luciferase assays (Botvinnik et al., 2010). 


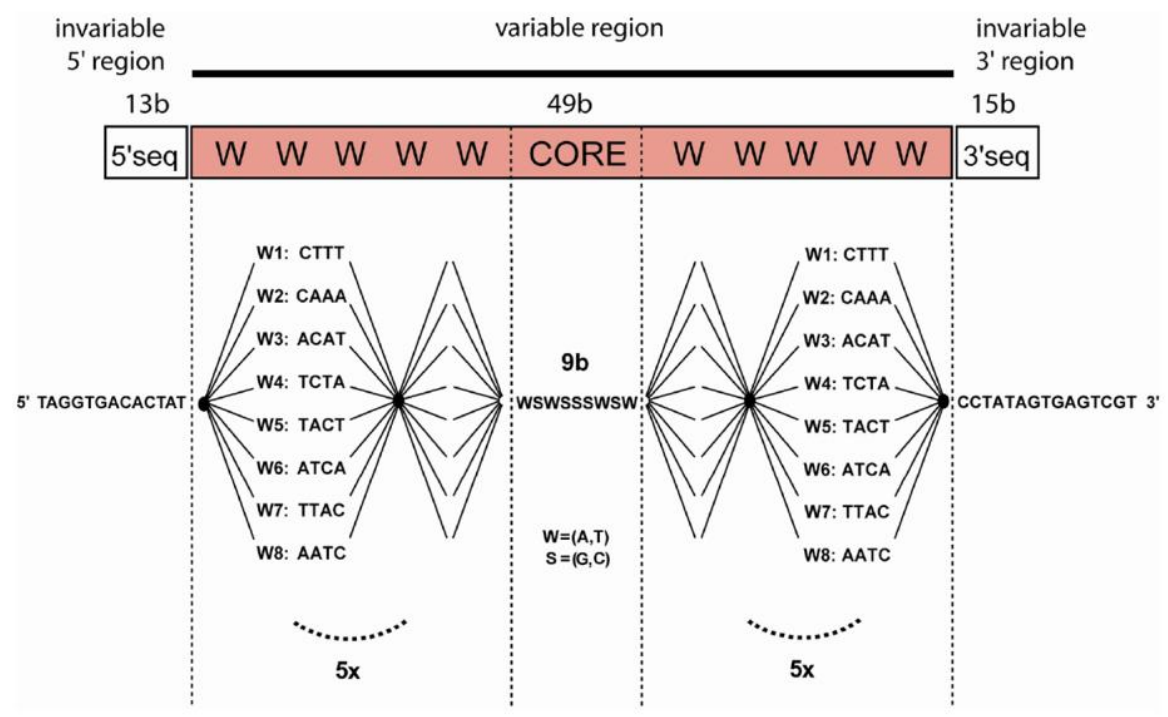

Figure 4: Structure of EXTs.

Each EXT contains a $5^{\prime}$ and $3^{\prime}$ invariable region and a 49-nucleotide variable region of ten 4-nucleotide "words" (W) and a 9-nucleotide core sequence. The upper part shows the random distribution of the eight "words" to the 10 positions during the EXT synthesis. The resulting complexity of "word" and core sequence combinations is $\sim 5.5 \times 10^{11}$. Adapted from Botvinnik and Rossner, 2012. 


\subsection{Aim of study}

GPCRs are essential modulators of many biological processes, which make them to key targets for a variety of therapeutic drugs. An important aspect within the drug discovery process is the potential property of compounds to modulate multiple targets. In the past, this polypharmacology was seen as disadvantage responsible for adverse side effects. However, over time it was shown that some drug classes exhibiting polypharmacology have better clinical efficacy. Antipsychotics represent such a class of drugs characterised by a complex pharmacology targeting multiple GPCRs for the treatment of psychiatric diseases. Approaches for profiling drug actions causing polypharmacological effects in a biological system may reveal desired targets and downstream signalling cues responsible for therapeutic effects (on-target \& pathway effects) as well as targets and pathway activities producing adverse side effects (off-target \& pathway effects). Furthermore, over the past decades it became clear that GPCR signalling is highly complex inducing G protein-dependent and independent signalling pathways. Methods to measure these complex GPCR signalling networks will lead to a better understanding of physiological effects caused by GPCR targeting drugs.

The aim of this thesis is to develop a multiplexed GPCR profiling system to simultaneously monitor multiple selected events in living cells, both at the level of receptor activation and downstream cellular signalling. Firstly, the thesis included the development of a multiplexed GPCR activation assay to assess receptor activities within a matrix of multiple GPCRs and ligands. A selection of GPCRs was adapted to the split TEV technique and connected to EXT barcode reporters to monitor receptor activation of multiple GPCRs by several ligands in parallel in one experiment. A major application of the developed assay was to generate activity profiles induced by compounds corresponding to endogenous neurotransmitters and hormones. Further, the assay was applied to profile the actions of clinically approved antipsychotic drugs and other antagonists. In the second part a multiplexed GPCR signalling assay was designed to monitor simultaneously multiple cellular events of GPCR mediated downstream signalling. For this an EXT reporter library encoding a variety of different cis-regulatory elements was used to assess signalling profiles of activated GPCRs at the level of transcription factor activity. 


\section{Materials}

\subsection{Equipment}

Arium 611 Water Purification System

Biophotometer

Picodrop Spektrophotometer

Vortex Genie 2

Mini Centrifuge Model sprout

Heraeus Centrifuge Fresco 17

Heraeus Megafuge 16

Thermomixer BioShake iQ

Thermocycler T3000

Thermocycler TGradient

Electrophoresis power supply

UV Gel documentation system

Genepulser Xcell Elektroporationsgerät

Ultra-low temperature lab freezer U725

\section{Cell culture}

Hera Cell incubator

Hera Safe Workbench

Nalgene freezing Container "Mr. Frosty"

Microscope Axiovert 25

\section{Luciferase measurements}

Tumbling Table WT17

Microplate Reader Mithras LB 940

32-Channel Luminometer LumiCycle 32
Sartorius

Eppendorf

Picodrop Limited

Bender + Hobein

Biozym

Thermo Fisher Scientific

Thermo Fisher Scientific

Biometra

Biometra

Biometra

Pharmacia LKB

INTAS

BioRad

New Brunswick Scientific

Thermo Fisher Scientific

Thermo Fisher Scientific

Sigma-Aldrich

Zeiss

Biometra

Berthold Technologies

ActiMetrics

Ion Torrent

Ion Torrent 
Ion Proton

Ion Torrent Server

Minifuge

Qubit 2.0 Fluorometer

UV Airclean Workstation
Ion Torrent

Ion Torrent

Ion Torrent

Invitrogen

LTF Labortechnik

\section{Software}

Microsoft Windows Professional 7

Microsoft

Microsoft Office 2007

Microsoft

Acrobat Reader 9.5

Adobe

Illustrator CS5

Adobe

Photoshop CS5

Adobe

InDesign CS5

Adobe

Lasergene 8.0

DNA Star Inc.

MicroWin 2000

Berthold Technologies

ImageJ

Freeware

R Version 2.15.0

Freeware

R-studio Version 0.99.484

Freeware

Tinn-R Editor Version 2.3.7.1

Freeware

Lumicycle Version 1.4

ActiMetrics

ChemSketch

ACDlabs

Zotero

\subsection{Chemicals and consumables}

All chemical reagents were purchased from Thermo Scientific, Sigma-Aldrich, Merck, Roche and Promega unless stated otherwise. The cell culture media and supplements were ordered from Thermo Scientific, Lonza or Sigma-Aldrich. General consumables were purchased from Eppendorf, Falcon, Sarstedt, Nunc, Nalgene, Biorad and Gilson. 


\subsubsection{GPCR ligands}

Aripiprazole

[Arg8]-Vasopressin acetate salt

Asenapine maleate

Dopamine-hydrochloride

( \pm )-Epinephrine hydrochloride

Histamine dihydrochloride

(-)-Isoproterenol hydrochloride

L-Norepinephrine hydrochloride

Paliperidone

(士)-Propranolol hydrochloride

Serotonin hydrochloride

Somatostatin

UNC0006

\subsubsection{Commercial kits}

RNeasy Mini Kit

RNase free DNase Kit

NucleoSpin Plasmid Quick Pure

NucleoBond PC100 Midiprep

NucleoBond PC500 Maxiprep

NucleoSpin Gel and PCR Clean-up

pGEM-T Vector System

\subsubsection{NGS Reagents \& Chips}

Qubit dsDNA HS Assay Kit

Ion PGM Template OT2 200 Kit

Ion PGM Sequencing 200 Kit v2

Ion PGM Hi-Q' ${ }^{\text {TM }}$ OT2 Kit

Ion PGM Hi-Q ${ }^{\text {TM }}$ Sequencing Kit

Ion $\mathrm{PI}^{\mathrm{TM}}$ Template OT2 200 Kit v3
Toronto Research Chemical

Sigma-Aldrich

Tocris

Sigma-Aldrich

Sigma-Aldrich

Tocris

Sigma-Aldrich

Sigma-Aldrich

Sigma-Aldrich

Sigma-Aldrich

Tocris

Sigma-Aldrich

Lead Discovery Center

Qiagen

Qiagen

Macherey-Nagel

Macherey-Nagel

Macherey-Nagel

Macherey-Nagel

Promega

Invitrogen

Ion Torrent

Ion Torrent

Ion Torrent

Ion Torrent

Ion Torrent 
Ion $\mathrm{PI}^{\mathrm{TM}}$ Sequencing $200 \mathrm{Kit} \mathrm{v} 3$

Ion Sphere Quality Control Kit

Dynabeads MyOne Streptavidin C1

DNA LoBind Tubes (1.5 ml)

Ion 314 Chip Kit v2

Ion 318 Chip Kit v2

Ion $\mathrm{PI}^{\mathrm{TM}}$ Chip Kit v2

\subsubsection{Enzymes}

HotStarTaq Plus DNA Polymerase

PWO Polymerase

Pfu Ultra Advanced DNA Polymerase

Pfu Turbo Polymerase

BP Clonase II

LR Clonase II

LR Clonase II Plus

SuperScript III Reverse Transcriptase

RQ1 DNase

Alkaline Phosphatase

Restriction enzymes
Ion Torrent

Ion Torrent

Invitrogen

Eppendorf

Ion Torrent

Ion Torrent

Ion Torrent

Qiagen

Roche

Stratagene

Stratagene

Thermo Fisher Scientific

Thermo Fisher Scientific

Thermo Fisher Scientific

Thermo Fisher Scientific

Promega

Roche

New England Biolabs

\subsection{Eukaryotic cell lines}

\section{PC12 tetOFF}

Rat adrenal pheochomocytoma cell line expressing tetracycline regulated transactivator tTA (Clonetech) (Greene and Tischler, 1976).

\section{U2OS}

Human osteosarcoma cells (American tissue culture collection, ATCC) (Pontén and Saksela, 1967).

\section{$\mathrm{CHO}$}

Chinese Hamster Ovary cells (Puck et al., 1958). 


\subsection{Bacterial strains}

Escherichia coli transformation competent cells:

MegaX DH10B electro-competent cells Thermo Fisher Scientific

One Shot Mach1 chemical-competent cells

Thermo Fisher Scientific

One Shot ccdB survival 2 T1R chemical-competent cells

Thermo Fisher Scientific

DH5 $\alpha$ chemical-competent cells

Self-made

Mach1 chemical-competent cells

Self-made

\subsection{Buffers and solutions}

\subsubsection{Molecular biology solutions}

TAE (50x)

2M Tris-Base

$50 \mathrm{mM} \quad$ EDTA

Adjust with glacial acetic acid to $\mathrm{pH} 8$ and with $\mathrm{H}_{2} \mathrm{O}$ to 1 .

TE (Tris-EDTA)

$10 \mathrm{mM} \quad$ Tris- $\mathrm{HCl}, \mathrm{pH} 7.5$

$1 \mathrm{mM} \quad$ EDTA

10x DNA Orange loading dye

$50 \% \quad$ Glycerol

$0.1 \% \quad$ Orange $\mathrm{G}$

49.9\% TAE buffer $1 x$

10x GelRed/Orange loading buffer

$90 \% 10 x$ DNA Orange loading dye

$10 \% \quad$ GelRed (from a 1:100 dilution of the $10000 x$ stock)

\section{Ethidiumbromid}

$1 \mathrm{mg} / \mathrm{ml} \quad \mathrm{EtBr} 1 \%$ in $\mathrm{H}_{2} \mathrm{O}$ 


\section{LB-medium (Luria and Bertani medium)}

$0.5 \% \quad$ Yeast extract

1\% Bacto-Peptone $\mathrm{pH} 7.5$

$1 \% \quad \mathrm{NaCl}$

Low-salt LB-medium

0.5\% Yeast extract

1\% $\quad$ Bacto-Peptone $\mathrm{pH} 7.5$

$0.5 \% \quad \mathrm{NaCl}$

LB/Amp: 200 g/ml f. c. Ampicillin in LB-medium

LB/Kan: $50 \mu \mathrm{g} / \mathrm{ml} \mathrm{f.} \mathrm{c.} \mathrm{Kanamycin} \mathrm{in} \mathrm{LB-medium}$

LB/Zeo: $35 \mu \mathrm{g} / \mathrm{ml}$ f. c. Zeocin in low-salt LB-medium

LB/Cm: $25 \mu \mathrm{g} / \mathrm{ml}$ f. c. Chloramphenicol in LB-medium

LB/Genta: $50 \mu \mathrm{g} / \mathrm{ml} \mathrm{f.} \mathrm{c.} \mathrm{Gentamicin} \mathrm{in} \mathrm{LB-medium}$

\section{SOC-Medium}

$\begin{array}{ll}0.5 \% & \text { Yeast extract } \\ 2 \% & \text { Bacto-Peptone } \\ 20 \mathrm{mM} & \text { Glucose } \\ 10 \mathrm{mM} & \mathrm{NaCl} \\ 2.5 \mathrm{mM} & \mathrm{KCl} \\ 10 \mathrm{mM} & \mathrm{MgSO}_{4}\end{array}$

LB-Agar plates

0.5\% Yeast extract

1\% $\quad$ Bacto-Peptone $\mathrm{pH} 7.5$

$1 \% \quad \mathrm{NaCl}$

1.5\% Agar

Autoclave and after cooling add antibiotics (final concentration like LB-media). 
For blue-white screening add:

$200 \mu \mathrm{g} / \mathrm{ml} \quad$ Ampicillin

$15 \mu \mathrm{g} / \mathrm{ml} \quad$ IPTG (Isopropyl- $\beta$-D-thiogalactopyranoside)

$35 \mu \mathrm{g} / \mathrm{ml} \quad X$-gal (5-bromo-4-chloro-3-indolyl- $\beta$-D-galactopyranoside)

Low-salt LB-Agar plates

0.5\% Yeast extract

1\% Bacto-Peptone $\mathrm{pH} 7.5$

$0.5 \% \quad \mathrm{NaCl}$

1.5\% Agar

Autoclave and after cooling add antibiotics (final concentration like LB-media).

\subsubsection{Solutions for luciferase assay}

\section{Lysis buffer}

$5 x$ Passive lysis buffer

Dilute buffer in $\mathrm{ddH}_{2} \mathrm{O}$.

Firefly luciferase assay buffer (Gaunitz and Papke 1998)

$\begin{array}{ll}20 \mathrm{mM} & \text { Tricine } \\ 1.07 \mathrm{mM} & \left(\mathrm{MgCO}_{3}\right) 4 * \mathrm{Mg}(\mathrm{OH})_{2} * 5 \mathrm{H}_{2} \mathrm{O} \\ 2.67 \mathrm{mM} & \mathrm{MgSO}_{4} \\ 0,1 \mathrm{mM} & \text { EDTA } \\ 33.3 \mathrm{mM} & \text { DTT } \\ 270 \mu \mathrm{M} & \text { Coenzyme A } \\ 470 \mu \mathrm{M} & \text { D-Luciferin, free base } \\ 530 \mu \mathrm{M} & \text { ATP }\end{array}$

For dissolving of magnesium carbonate titrate the $\mathrm{pH}$ with $\mathrm{HCl}$ until the solution is clear. Then adjust the $\mathrm{pH}$ to 7.8 with $\mathrm{NaOH}$. Add luciferin and ATP at last and control the $\mathrm{pH}$. The buffer is stored at $-20^{\circ} \mathrm{C}$ in the dark and thawed at room temperature. 


\section{Renilla luciferase assay buffer}

Prepare $\mathrm{KxPO}_{4}(\mathrm{pH} 5.1)$ solution: adjust the $\mathrm{pH}$ of $1 \mathrm{M} \mathrm{KH}_{2} \mathrm{PO}_{4}$ solution to 5.1 using $2 \mathrm{M}$ $\mathrm{KOH}$.

$\begin{array}{ll}1.1 \mathrm{M} & \mathrm{NaCl} \\ 2.2 \mathrm{mM} & \mathrm{Na}_{2} \text {-EDTA } \\ 0.22 \mathrm{M} & \mathrm{KxPO}_{4}(\mathrm{pH} 5.1) \\ 0.44 \mathrm{mg} / \mathrm{ml} & \mathrm{BSA} \\ 1.3 \mathrm{mM} & \mathrm{NaN}_{3}\end{array}$

Adjust the $\mathrm{pH}$ to 5.0 with $\mathrm{KOH}$. Then add $1.43 \mathrm{mM}$ Coelenterazine (dissolved in $100 \% \mathrm{EtOH})$. The buffer is stored at $-20^{\circ} \mathrm{C}$ in the dark and thawed at room temperature.

\subsubsection{Solutions and media for cell culture}

PBS 10x (Phosphate buffered saline)

1.7 $\mathrm{M} \quad \mathrm{NaCl}$

$34 \mathrm{mM} \quad \mathrm{KCl}$

$40 \mathrm{mM} \quad \mathrm{Na}_{2} \mathrm{HPO}_{4} * 2 \mathrm{H}_{2} \mathrm{O}$

$18 \mathrm{mM} \quad \mathrm{KH}_{2} \mathrm{PO}_{4}$

Adjust $\mathrm{pH}$ to 7.2 with $1 \mathrm{M} \mathrm{NaOH}$.

\section{PLL 250x}

$5 \mathrm{mg} / \mathrm{ml} \quad$ Poly-L-Lysine in $\mathrm{H}_{2} \mathrm{O}$

Dilute in $\mathrm{dd}_{2} \mathrm{O}$. Final concentration: $0.02 \mathrm{mg} / \mathrm{ml}$.

\section{Trypsin 10x}

$5 \mathrm{~g} / \mathrm{l} \quad$ Trypsin

Dilute in PBS.

Penicillin-Streptomycin (PenStrep)

10000 U/ml Potassium Penicillin

$10000 \mu \mathrm{g} / \mathrm{ml}$ Streptomycin Sulfate 
2x Freezing medium for eukaryotic cell lines

$\begin{array}{ll}40 \% & \text { DMEM } \\ 40 \% & \text { FBS } \\ 20 \% & \text { DMSO }\end{array}$

PC12tetOFF maintenance medium

$\begin{array}{ll}500 \mathrm{ml} & \text { DMEM (1g/l Glucose) } \\ 10 \% & \text { Dialyzed FBS } \\ 1 \% & \text { NEAA } \\ 1 \% & \text { GlutaMAX } \\ 1 \% & \text { PenStrep }\end{array}$

PC12tetOFF assay medium

$500 \mathrm{ml}$ DMEM (1g/l Glucose)

1\% Dialysed FBS

$0.1 \% \quad$ NEAA

1\% GlutaMAX

1\% PenStrep

U2OS maintenance medium

$\begin{array}{ll}500 \mathrm{ml} & \text { McCoy's 5A + GlutaMax } \\ 10 \% & \text { Dialyzed FBS } \\ 1 \% & \text { NEAA } \\ 1 \% & \text { PenStrep }\end{array}$

U2OS assay medium

$\begin{array}{ll}500 \mathrm{ml} & \text { McCoy's 5A + GlutaMax } \\ 0.1 \% & \text { Dialysed FBS } \\ 0.1 \% & \text { NEAA } \\ 1 \% & \text { PenStrep }\end{array}$




\section{CHO maintenance medium}

500ml F12 + GlutaMax

10\% Dialyzed FBS

$1 \%$ NEAA

1\% PenStrep

\section{$\mathrm{CHO}$ assay medium}

500ml F12 + GlutaMax

0.1\% Dialysed FBS

$0.1 \% \quad$ NEAA

1\% PenStrep

\subsection{Oligonucleotides}

Oligonucleotides were made by the AGCTlab (facility of the department of neurobiology) of the MPI of Experimental Medicine in Göttingen or purchased from Eurofins in Munich. 


\subsection{Plasmids}

Table 1: List of plasmids.

\begin{tabular}{lll}
\multicolumn{2}{l}{ Gateway backbone vectors } \\
V9 & pDONR207 & GentaR \\
V40 & pDONR221 & KanR \\
V45 & pDONR-Zeo & ZeoR \\
V881 & pDONR_P1-P4 & KanR \\
V882 & pDONR_P4r-P2 & KanR \\
V883 & pDONR_P4r-P3r & KanR \\
V884 & pDONR_P3-P2 & KanR \\
V101 & pTag4C_ST_X-V2R-NTEV-tevS-GV_DEST & KanR \\
V582 & pCDNA3.1_Zeo_X-CTEV-2HA_DEST & AmpR \\
V84 & pGL4.16_X_luc2_Hygro_DEST & AmpR \\
V825 & pAAVspace_DEST_X_luc2_WPRE & AmpR \\
V288 & pEF5/FRT_X-V5_DEST & AmpR
\end{tabular}

\section{GPCR entry vectors}

V202

V243

V203

V206

V246

V207

V247

V211

V212

V213

V214

V242

V215

V216

V217

V240

V218

V241

V219

V173

V174

V175

V191

V177

V232

V230

V231

V234
pENTR207_hHTR1A-Cop

pENTR-Zeo_HA-Flag-hHTR1A-Cop

pENTR207_hHTR1B-Cop

pENTR207_hHTR2A-var1-Cop

pENTR-Zeo_HOOK-hHTR2A-var1-Cop

pENTR207_hHTR2B-Cop

pENTR-Zeo_HOOK-hHTR2B-Cop

pENTR207_hHTR4-vara-Cop

pENTR207_hHTR5A-Cop

pENTR207_hHTR6-var-Cop

pENTR-Zeo_hHTR7-vara-Cop

pENTR-Zeo_HA-Flag-hHTR7-vara-Cop

pENTR207_hDRD1-Cop

pENTR207_hDRD2-var2-Cop

pENTR207_hDRD3-vara-Cop

pENTR-Zeo_HA-Flag-hDRD3-vara-Cop

pENTR-Zeo_hDRD4-Cop

pENTR-Zeo_HA-Flag-hDRD4-Cop

pENTR207_hDRD5-Cop

pENTR-Zeo_hADRA1A-var2-Cop

pENTR-Zeo_hADRA2B-Cop

pENTR-Zeo_hADRA2C-Cop

pENTR207_hADRB2-Cop

pENTR-Zeo_hADRB3-Cop

pENTR207_hHRH1-var4-Cop

pENTR221_hAVPR1A-Cop

pENTR207_hAVPR2-var1-Cop

pENTR207_hSSTR1-Cop
GentaR

ZeoR

GentaR

GentaR

ZeoR

GentaR

ZeoR

GentaR

GentaR

GentaR

ZeoR

ZeoR

GentaR

GentaR

GentaR

ZeoR

ZeoR

ZeoR

GentaR

ZeoR

ZeoR

ZeoR

GentaR

ZeoR

GentaR

KanR

GentaR

GentaR 
ZeoR

\section{GPCRs expression vectors (split TEV)}

$\begin{array}{lll}\text { V434 } & \text { pTag4C_HA-Flag-hHTR1A-V2R-NTEV-tevS-GV } & \text { KanR } \\ \text { V436 } & \text { pTag4C_hHTR1B-V2R-NTEV-tevS-GV } & \text { KanR } \\ \text { V441 } & \text { pTag4C_HOOK-hHTR2A-var1-V2R-NTEV-tevS-GV } & \text { KanR } \\ \text { V444 } & \text { pTag4C_hHTR4-vara-V2R-NTEV-tevS-GV } & \text { KanR } \\ \text { V445 } & \text { pTag4C_hHTR5A-V2R-NTEV-tevS-GV } & \text { KanR } \\ \text { V446 } & \text { pTag4C_hHTR6-var-V2R-NTEV-tevS-GV } & \text { KanR } \\ \text { V447 } & \text { pTag4C_hHTR7-vara-V2R-NTEV-tevS-GV } & \text { KanR } \\ \text { V448 } & \text { pTag4C_HA-Flag-hHTR7-vara-V2R-NTEV-tevS-GV } & \text { KanR } \\ \text { V406 } & \text { pTag4C_hDRD1-V2R-NTEV-tevS-GV } & \text { KanR } \\ \text { V407 } & \text { pTag4C_hDRD2-var2-V2R-NTEV-tevS-GV } & \text { KanR } \\ \text { V412 } & \text { pTag4C_HA-Flag-hDRD3-vara-V2R-NTEV-tevS-GV } & \text { KanR } \\ \text { V413 } & \text { pTag4C_HA-Flag-hDRD4-V2R-NTEV-tevS-GV } & \text { KanR } \\ \text { V410 } & \text { pTag4C_hDRD5-V2R-NTEV-tevS-GV } & \text { KanR } \\ \text { V267 } & \text { pTag4C_hADRA1A-var2_V2R-NTEV-tevS-GV } & \text { KanR } \\ \text { V270 } & \text { pTag4C_hADRA2B_V2R-NTEV-tevS-GV } & \text { KanR } \\ \text { V272 } & \text { pTag4C_hADRA2C_V2R-NTEV-tevS-GV } & \text { KanR } \\ \text { V357 } & \text { pTag4C_hADRB2-V2R-NTEV-tevS-GV } & \text { KanR } \\ \text { V1097 } & \text { pTag4C_hADRB3-V2R-NTEV-tevS-GV } & \text { KanR } \\ \text { V286 } & \text { pTag4C_hHRH1-var4_V2R-NTEV-tevS-GV } & \text { KanR } \\ \text { V282 } & \text { pTag4C_hAVPR1A_V2R-NTEV-tevS-GV } & \text { KanR } \\ \text { V284 } & \text { pTag4C_hAVPR2-var1_V2R-NTEV-tevS-GV } & \text { KanR } \\ \text { V302 } & \text { pTag4C_hSSTR1_V2R-NTEV-tevS-GV } & \text { KanR } \\ \text { V306 } & \text { pTag4C_HOOK-hSSTR1_V2R-NTEV-tevS-GV } & \text { KanR } \\ \text { V303 } & \text { pTag4C_hSSTR2_V2R-NTEV-tevS-GV } & \text { KanR } \\ \text { V304 } & \text { pTag4C_hSSTR3_V2R-NTEV-tevS-GV } & \text { KanR } \\ \text { V305 } & \text { pTag4C_hSSTR5_V2R-NTEV-tevS-GV } & \text { KanR }\end{array}$

GPCRs expression vectors (V5 tag)

$\begin{array}{lll}\text { V463 } & \text { pEF5/FRT_HOOK-hHTR2A-var1-V5 } & \text { AmpR } \\ \text { V469 } & \text { pEF5/FRT_hHTR4-vara-V5 } & \text { AmpR } \\ \text { V415 } & \text { pEF5/FRT_DRD1-V5 } & \text { AmpR } \\ \text { V291 } & \text { pEF5/FRT_hADRA2C-V5 } & \text { AmpR } \\ \text { V1100 } & \text { pEF5/FRT_hADRB2-V5 } & \text { AmpR } \\ \text { V300 } & \text { pEF5/FRT_hHRH1-var4-V5 } & \text { AmpR } \\ \text { V311 } & \text { pEF5/FRT_HOOK-hSSTR1-V5 } & \text { AmpR } \\ \text { V307 } & \text { pEF5/FRT_hSSTR1-V5 } & \text { AmpR }\end{array}$

Split TEV 


\begin{tabular}{lll}
\multicolumn{2}{l}{ Luciferase vectors } \\
V1050 & pGL4.16-10xUAS-CMV-E00370-S074_FFLuc2 & AmpR \\
V66 & phRL-TK & KanR \\
V67 & phRL-SV40 & KanR \\
V68 & phRL-CMV & KanR
\end{tabular}

\section{EXT split TEV sensors}

\begin{tabular}{|c|c|c|}
\hline V1050 & pGL4.16-10xUAS-CMV-E00369-S074 & AmpR \\
\hline V1050 & pGL4.16-10xUAS-CMV-E00370-S074 & AmpR \\
\hline V1050 & pGL4.16-10xUAS-CMV-E00371-S074 & AmpR \\
\hline V1050 & pGL4.16-10xUAS-CMV-E00372-S074 & AmpR \\
\hline V1050 & pGL4.16-10xUAS-CMV-E00373-S074 & AmpR \\
\hline V1050 & pGL4.16-10xUAS-CMV-E00374-S074 & AmpR \\
\hline V1050 & pGL4.16-10xUAS-CMV-E00376-S074 & AmpR \\
\hline V1050 & pGL4.16-10xUAS-CMV-E00377-S074 & AmpR \\
\hline V1050 & pGL4.16-10xUAS-CMV-E00382-S074 & AmpR \\
\hline V1050 & pGL4.16-10xUAS-CMV-E00383-S074 & AmpR \\
\hline V1050 & pGL4.16-10xUAS-CMV-E00385-S074 & AmpR \\
\hline V1050 & pGL4.16-10xUAS-CMV-E00386-S074 & AmpR \\
\hline V1050 & pGL4.16-10xUAS-CMV-E00387-S074 & AmpR \\
\hline V1050 & pGL4.16-10xUAS-CMV-E00388-S074 & AmpR \\
\hline V1050 & pGL4.16-10xUAS-CMV-E00389-S074 & AmpR \\
\hline V1050 & pGL4.16-10xUAS-CMV-E00390-S074 & AmpR \\
\hline V1050 & pGL4.16-10xUAS-CMV-E00391-S074 & AmpR \\
\hline V1050 & pGL4.16-10xUAS-CMV-E00393-S074 & AmpR \\
\hline V1050 & pGL4.16-10xUAS-CMV-E00395-S074 & AmpR \\
\hline V1050 & pGL4.16-10xUAS-CMV-E00396-S074 & AmpR \\
\hline V1050 & pGL4.16-10xUAS-CMV-E00397-S074 & AmpR \\
\hline V1050 & pGL4.16-10xUAS-CMV-E00398-S074 & AmpR \\
\hline V1050 & pGL4.16-10xUAS-CMV-E00399-S074 & AmpR \\
\hline V1050 & pGL4.16-10xUAS-CMV-E00400-S074 & AmpR \\
\hline V1050 & pGL4.16-10xUAS-CMV-E00401-S074 & AmpR \\
\hline V1050 & pGL4.16-10xUAS-CMV-E00402-S074 & AmpR \\
\hline V1050 & pGL4.16-10xUAS-CMV-E00403-S074 & AmpR \\
\hline V1050 & pGL4.16-10xUAS-CMV-E00404-S074 & AmpR \\
\hline V1050 & pGL4.16-10xUAS-CMV-E00405-S074 & AmpR \\
\hline V1050 & pGL4.16-10xUAS-CMV-E00407-S074 & AmpR \\
\hline V1050 & pGL4.16-10xUAS-CMV-E00408-S074 & $A m p R$ \\
\hline V1050 & pGL4.16-10xUAS-CMV-E00410-S074 & $A m p R$ \\
\hline V1050 & pGL4.16-10xUAS-CMV-E00411-S074 & $A m p R$ \\
\hline V1050 & pGL4.16-10xUAS-CMV-E00412-S074 & $A m p R$ \\
\hline V1050 & pGL4.16-10xUAS-CMV-E00413-S074 & AmpR \\
\hline V1050 & pGL4.16-10xUAS-CMV-E00414-S074 & AmpR \\
\hline V1050 & pGL4.16-10xUAS-CMV-E00415-S074 & $A m p R$ \\
\hline V1050 & pGL4.16-10xUAS-CMV-E00418-S074 & $A m p R$ \\
\hline V1050 & pGL4.16-10xUAS-CMV-E00419-S074 & AmpR \\
\hline V1050 & pGL4.16-10xUAS-CMV-E00420-S074 & AmpR \\
\hline
\end{tabular}




\begin{tabular}{|c|c|c|}
\hline V1050 & pGL4.16-10xUAS-CMV-E00423-S074 & AmpR \\
\hline V1050 & pGL4.16-10xUAS-CMV-E00424-S074 & AmpR \\
\hline V1050 & pGL4.16-10xUAS-CMV-E00425-S074 & AmpR \\
\hline V1050 & pGL4.16-10xUAS-CMV-E00427-S074 & AmpR \\
\hline V1050 & pGL4.16-10xUAS-CMV-E00428-S074 & AmpR \\
\hline V1050 & pGL4.16-10xUAS-CMV-E00429-S074 & AmpR \\
\hline V1050 & pGL4.16-10xUAS-CMV-E00430-S074 & AmpR \\
\hline V1050 & pGL4.16-10xUAS-CMV-E00431-S074 & AmpR \\
\hline V1050 & pGL4.16-10xUAS-CMV-E00432-S074 & AmpR \\
\hline V1050 & pGL4.16-10xUAS-CMV-E00434-S074 & AmpR \\
\hline V1050 & pGL4.16-10xUAS-CMV-E00436-S074 & AmpR \\
\hline V1050 & pGL4.16-10xUAS-CMV-E00439-S074 & AmpR \\
\hline V1050 & pGL4.16-10xUAS-CMV-E00440-S074 & AmpR \\
\hline V1050 & pGL4.16-10xUAS-CMV-E00441-S074 & AmpR \\
\hline V1050 & pGL4.16-10xUAS-CMV-E00442-S074 & AmpR \\
\hline V1050 & pGL4.16-10xUAS-CMV-E00443-S074 & AmpR \\
\hline V1050 & pGL4.16-10xUAS-CMV-E00448-S074 & AmpR \\
\hline V1050 & pGL4.16-10xUAS-CMV-E00450-S074 & AmpR \\
\hline V1050 & pGL4.16-10xUAS-CMV-E00451-S074 & AmpR \\
\hline V1050 & pGL4.16-10xUAS-CMV-E00452-S074 & AmpR \\
\hline V1050 & pGL4.16-10xUAS-CMV-E00455-S074 & AmpR \\
\hline V1050 & pGL4.16-10xUAS-CMV-E00456-S074 & AmpR \\
\hline V1050 & pGL4.16-10xUAS-CMV-E00457-S074 & AmpR \\
\hline V1050 & pGL4.16-10xUAS-CMV-E00459-S074 & AmpR \\
\hline V1050 & pGL4.16-10xUAS-CMV-E00460-S074 & AmpR \\
\hline V1050 & pGL4.16-10xUAS-CMV-E00461-S074 & AmpR \\
\hline V1050 & pGL4.16-10xUAS-CMV-E00463-S074 & AmpR \\
\hline \multicolumn{3}{|c|}{ Calibrator EXT reporters } \\
\hline V1093 & pGL3-5xUAS-TK-E00055-S073 & AmpR \\
\hline V1093 & pGL3-5xUAS-TK-E00056-S073 & AmpR \\
\hline V1093 & pGL3-5xUAS-TK-E00057-S073 & AmpR \\
\hline V1093 & pGL3-5xUAS-TK-E00058-S073 & AmpR \\
\hline V1093 & pGL3-5xUAS-TK-E00059-S073 & AmpR \\
\hline V1093 & pGL3-5xUAS-TK-E00060-S073 & AmpR \\
\hline V1093 & pGL3-5xUAS-TK-E00061-S073 & AmpR \\
\hline V1093 & pGL3-5xUAS-TK-E00062-S073 & $A m p R$ \\
\hline V1093 & pGL3-5xUAS-TK-E00063-S073 & $A m p R$ \\
\hline V1093 & pGL3-5xUAS-TK-E00064-S073 & $A m p R$ \\
\hline V1093 & pGL3-5xUAS-TK-E00065-S073 & AmpR \\
\hline V1093 & pGL3-5xUAS-TK-E00066-S073 & $A m p R$ \\
\hline V1093 & pGL3-5xUAS-TK-E00067-S073 & $A m p R$ \\
\hline V1093 & pGL3-5xUAS-TK-E00068-S073 & AmpR \\
\hline V1093 & pGL3-5xUAS-TK-E00069-S073 & $A m p R$ \\
\hline V1244 & pAAV-sEGFP-MLP-E00250-S072 & AmpR \\
\hline V1244 & pAAV-sEGFP-MLP-E00252-S072 & $A m p R$ \\
\hline V1244 & pAAV-sEGFP-MLP-E00253-S072 & AmpR \\
\hline
\end{tabular}


AmpR

\section{EXT downstream signalling sensors}

\section{Cis-EXT reporters}

V1296

V1296

V1296

V1397

V1397

V1397

V1339

V1339

V1339

V1342

V1342

V1342

V1343

V1343

V1343

V1340

V1340

V1340

V1344

V1344

V1344

V1253

V1253

V1345

V1345

V1345

V1400

V1400

V1400

V1346

V1346

V1346

V1399

V1399

V1399

V1347

V1347

V1347

V1348
PAAV-AARE-MLP-E01001-S001

pAAV-AARE-MLP-E01002-S001

PAAV-AARE-MLP-E01003-S001

pAAV-AP1-v1-MLP-E01292-S060

pAAV-AP1-V1-MLP-E01293-S060

pAAV-AP1-v1-MLP-E01294-S060

pAAV-AP1-V2-MLP-E01006-S002

pAAV-AP1-v2-MLP-E01007-S002

PAAV-AP1-v2-MLP-E01008-S002

pAAV-ARE-V1-MLP-E01015-S004

pAAV-ARE-v1-MLP-E01016-S004

PAAV-ARE-v1-MLP-E01017-S004

pAAV-ARE-V2-MLP-E01018-S005

pAAV-ARE-v2-MLP-E01019-S005

PAAV-ARE-V2-MLP-E01020-S005

PAAV-AR-RE-MLP-E01011-S003

PAAV-AR-RE-MLP-E01013-S003

pAAV-AR-RE-MLP-E01014-S003

PAAV-CEBP-RE-v1-MLP-E01026-S006

PAAV-CEBP-RE-v1-MLP-E01027-S006

PAAV-CEBP-RE-v1-MLP-E01028-S006

PAAV-CRE-MLP-E00482-S066

PAAV-CRE-MLP-E00491-S066

pAAV-E2F-RE-v1-MLP-E01036-S008

pAAV-E2F-RE-v1-MLP-E01037-S008

pAAV-E2F-RE-v1-MLP-E01038-S008

pAAV-E2F-RE-V2-MLP-E00249-S063

pAAV-E2F-RE-v2-MLP-E01309-S063

pAAV-E2F-RE-v2-MLP-E01310-S063

pAAV-E2F-RE-V3-MLP-E01041-S009

pAAV-E2F-RE-V3-MLP-E01042-S009

PAAV-E2F-RE-v3-MLP-E01043-S009

pAAV-E-box-MLP-E01304-S062

pAAV-E-box-MLP-E01306-S062

pAAV-E-box-MLP-E01307-S062

pAAV-EGR1-RE-V1-MLP-E01045-S010

pAAV-EGR1-RE-v1-MLP-E01046-S010

PAAV-EGR1-RE-v1-MLP-E01047-S010

pAAV-EGR1-RE-V2-MLP-E01050-S011
AmpR

AmpR

AmpR

AmpR

AmpR

AmpR

AmpR

AmpR

AmpR

AmpR

AmpR

AmpR

AmpR

AmpR

AmpR

AmpR

AmpR

AmpR

AmpR

AmpR

AmpR

AmpR

AmpR

AmpR

AmpR

AmpR

AmpR

AmpR

AmpR

AmpR

AmpR

AmpR

AmpR

AmpR

AmpR

AmpR

AmpR

AmpR

AmpR 


\begin{tabular}{|c|c|c|}
\hline V1348 & pAAV-EGR1-RE-v2-MLP-E01051-S011 & AmpR \\
\hline V1348 & pAAV-EGR1-RE-v2-MLP-E01052-S011 & AmpR \\
\hline V1349 & pAAV-ELK1-RE-MLP-E01054-S012 & AmpR \\
\hline V1349 & pAAV-ELK1-RE-MLP-E01055-S012 & AmpR \\
\hline V1349 & pAAV-ELK1-RE-MLP-E01056-S012 & AmpR \\
\hline V1350 & pAAV-ER-RE-MLP-E01063-S013 & AmpR \\
\hline V1350 & pAAV-ER-RE-MLP-E01064-S013 & AmpR \\
\hline V1350 & PAAV-ER-RE-MLP-E01065-S013 & AmpR \\
\hline V1351 & PAAV-ERSE-MLP-E01066-S014 & AmpR \\
\hline V1351 & pAAV-ERSE-MLP-E01068-S014 & AmpR \\
\hline V1351 & PAAV-ERSE-MLP-E01069-S014 & AmpR \\
\hline V1477 & pAAV-FOSe2-MLP-E01331-S105 & AmpR \\
\hline V1477 & pAAV-FOSe2-MLP-E01332-S105 & AmpR \\
\hline V1477 & pAAV-FOSe2-MLP-E01333-S105 & AmpR \\
\hline V1352 & pAAV-FOXO-RE-MLP-E01070-S015 & AmpR \\
\hline V1352 & pAAV-FOXO-RE-MLP-E01071-S015 & AmpR \\
\hline V1352 & pAAV-FOXO-RE-MLP-E01072-S015 & AmpR \\
\hline V1353 & pAAV-GATA-RE-v1-MLP-E01076-S016 & AmpR \\
\hline V1353 & pAAV-GATA-RE-v1-MLP-E01078-S016 & AmpR \\
\hline V1353 & pAAV-GATA-RE-v1-MLP-E01079-S016 & AmpR \\
\hline V1354 & pAAV-GATA-RE-v2-MLP-E01083-S017 & AmpR \\
\hline V1354 & pAAV-GATA-RE-v2-MLP-E01084-S017 & AmpR \\
\hline V1354 & pAAV-GATA-RE-v2-MLP-E01085-S017 & AmpR \\
\hline V1355 & pAAV-GLI-RE-MLP-E01086-S018 & AmpR \\
\hline V1355 & pAAV-GLI-RE-MLP-E01088-S018 & AmpR \\
\hline V1355 & pAAV-GLI-RE-MLP-E01089-S018 & AmpR \\
\hline V1356 & pAAV-GR-RE-MLP-E01092-S019 & AmpR \\
\hline V1356 & pAAV-GR-RE-MLP-E01093-S019 & AmpR \\
\hline V1356 & pAAV-GR-RE-MLP-E01094-S019 & AmpR \\
\hline V1357 & pAAV-HRE-MLP-E01098-S020 & AmpR \\
\hline V1357 & pAAV-HRE-MLP-E01100-S020 & AmpR \\
\hline V1357 & PAAV-HRE-MLP-E01101-S020 & AmpR \\
\hline V1358 & pAAV-HSE-MLP-E01105-S021 & AmpR \\
\hline V1358 & pAAV-HSE-MLP-E01106-S021 & AmpR \\
\hline V1358 & pAAV-HSE-MLP-E01108-S021 & AmpR \\
\hline V1359 & pAAV-IRS-MLP-E01113-S022 & AmpR \\
\hline V1359 & pAAV-IRS-MLP-E01114-S022 & AmpR \\
\hline V1359 & pAAV-IRS-MLP-E01116-S022 & AmpR \\
\hline V1360 & pAAV-ISRE-MLP-E01120-S023 & AmpR \\
\hline V1360 & pAAV-ISRE-MLP-E01121-S023 & AmpR \\
\hline V1360 & PAAV-ISRE-MLP-E01122-S023 & AmpR \\
\hline V1361 & pAAV-KLF4-RE-MLP-E01127-S024 & AmpR \\
\hline V1361 & pAAV-KLF4-RE-MLP-E01128-S024 & AmpR \\
\hline V1361 & pAAV-KLF4-RE-MLP-E01129-S024 & AmpR \\
\hline V1362 & pAAV-MRE-MLP-E01131-S025 & AmpR \\
\hline V1362 & pAAV-MRE-MLP-E01133-S025 & AmpR \\
\hline
\end{tabular}




\begin{tabular}{|c|c|c|}
\hline V1362 & pAAV-MRE-MLP-E01134-S025 & AmpR \\
\hline V1363 & pAAV-Myc-E-box-MLP-E01136-S026 & AmpR \\
\hline V1363 & pAAV-Myc-E-box-MLP-E01137-S026 & AmpR \\
\hline V1363 & pAAV-Myc-E-box-MLP-E01139-S026 & AmpR \\
\hline V1364 & pAAV-NANOG-RE-MLP-E01140-S027 & AmpR \\
\hline V1364 & pAAV-NANOG-RE-MLP-E01142-S027 & AmpR \\
\hline V1364 & pAAV-NANOG-RE-MLP-E01143-S027 & AmpR \\
\hline V1252 & pAAV-NFAT-RE-MLP-E00522-S067 & AmpR \\
\hline V1252 & pAAV-NFAT-RE-MLP-E00530-S067 & AmpR \\
\hline V1252 & pAAV-NFAT-RE-MLP-E00532-S067 & AmpR \\
\hline V1395 & pAAV-NFkB-RE-v1-MLP-E00244-S058 & AmpR \\
\hline V1395 & pAAV-NFkB-RE-v1-MLP-E00245-S058 & AmpR \\
\hline V1395 & pAAV-NFkB-RE-v1-MLP-E01284-S058 & AmpR \\
\hline V1401 & pAAV-NFkB-RE-v2-MLP-E01311-S065 & AmpR \\
\hline V1401 & pAAV-NFkB-RE-v2-MLP-E01312-S065 & AmpR \\
\hline V1401 & pAAV-NFkB-RE-v2-MLP-E01313-S065 & AmpR \\
\hline V1373 & pAAV-Notch-RE-MLP-E01188-S036 & AmpR \\
\hline V1373 & pAAV-Notch-RE-MLP-E01189-S036 & AmpR \\
\hline V1373 & pAAV-Notch-RE-MLP-E01190-S036 & AmpR \\
\hline V1476 & pAAV-Npas4-RE-MLP-E01324-S102 & AmpR \\
\hline V1476 & pAAV-Npas4-RE-MLP-E01325-S102 & AmpR \\
\hline V1476 & pAAV-Npas4-RE-MLP-E01326-S102 & AmpR \\
\hline V1475 & pAAV-NR4A1-RE-MLP-E01349-S109 & AmpR \\
\hline V1475 & pAAV-NR4A1-RE-MLP-E01350-S109 & AmpR \\
\hline V1475 & pAAV-NR4A1-RE-MLP-E01351-S109 & AmpR \\
\hline V1365 & pAAV-NRSE-MLP-E01145-S028 & AmpR \\
\hline V1365 & pAAV-NRSE-MLP-E01146-S028 & AmpR \\
\hline V1365 & pAAV-NRSE-MLP-E01147-S028 & AmpR \\
\hline V1367 & pAAV-OCT4-minP-MLP-E00191-S030 & AmpR \\
\hline V1367 & pAAV-OCT4-minP-MLP-E01155-S030 & AmpR \\
\hline V1367 & pAAV-OCT4-minP-MLP-E01156-S030 & AmpR \\
\hline V1366 & pAAV-OCT4-RE-MLP-E01149-S029 & AmpR \\
\hline V1366 & pAAV-OCT4-RE-MLP-E01152-S029 & AmpR \\
\hline V1366 & pAAV-OCT4-RE-MLP-E01153-S029 & AmpR \\
\hline V1396 & pAAV-p53-RE-v1-MLP-E01286-S059 & AmpR \\
\hline V1396 & pAAV-p53-RE-v1-MLP-E01287-S059 & AmpR \\
\hline V1396 & pAAV-p53-RE-v1-MLP-E01289-S059 & AmpR \\
\hline V1368 & pAAV-p53-RE-v2-MLP-E01165-S031 & AmpR \\
\hline V1368 & pAAV-p53-RE-v2-MLP-E01166-S031 & AmpR \\
\hline V1368 & pAAV-p53-RE-v2-MLP-E01167-S031 & AmpR \\
\hline V1369 & pAAV-RARE-v1-MLP-E01169-S032 & AmpR \\
\hline V1369 & pAAV-RARE-v1-MLP-E01170-S032 & AmpR \\
\hline V1369 & pAAV-RARE-v1-MLP-E01171-S032 & AmpR \\
\hline V1370 & pAAV-RARE-v2-MLP-E01172-S033 & AmpR \\
\hline V1370 & pAAV-RARE-v2-MLP-E01173-S033 & AmpR \\
\hline V1370 & pAAV-RARE-v2-MLP-E01174-S033 & AmpR \\
\hline
\end{tabular}




\begin{tabular}{|c|c|c|}
\hline V1371 & pAAV-RARE-v3-MLP-E01176-S034 & AmpR \\
\hline V1371 & pAAV-RARE-v3-MLP-E01177-S034 & AmpR \\
\hline V1371 & pAAV-RARE-v3-MLP-E01178-S034 & AmpR \\
\hline V1398 & pAAV-RB-RE-v1-MLP-E00265-S061 & AmpR \\
\hline V1398 & pAAV-RB-RE-v1-MLP-E01298-S061 & AmpR \\
\hline V1398 & pAAV-RB-RE-v1-MLP-E01299-S061 & AmpR \\
\hline V1372 & pAAV-RB-RE-v2-MLP-E01183-S035 & AmpR \\
\hline V1372 & pAAV-RB-RE-v2-MLP-E01184-S035 & AmpR \\
\hline V1372 & pAAV-RB-RE-v2-MLP-E01186-S035 & AmpR \\
\hline V1443 & pAAV-SARE-MLP-E01316-S071 & AmpR \\
\hline V1443 & pAAV-SARE-MLP-E01317-S071 & AmpR \\
\hline V1443 & pAAV-SARE-MLP-E01343-S071 & AmpR \\
\hline V1374 & pAAV-SMAD-RE-v1-MLP-E01191-S037 & AmpR \\
\hline V1374 & pAAV-SMAD-RE-v1-MLP-E01192-S037 & AmpR \\
\hline V1374 & pAAV-SMAD-RE-v1-MLP-E01193-S037 & AmpR \\
\hline V1375 & pAAV-SMAD-RE-v2-MLP-E01196-S038 & AmpR \\
\hline V1375 & pAAV-SMAD-RE-v2-MLP-E01197-S038 & AmpR \\
\hline V1375 & pAAV-SMAD-RE-v2-MLP-E01198-S038 & AmpR \\
\hline V1376 & pAAV-SMAD-RE-v3-MLP-E01201-S039 & AmpR \\
\hline V1376 & pAAV-SMAD-RE-v3-MLP-E01203-S039 & AmpR \\
\hline V1376 & pAAV-SMAD-RE-v3-MLP-E01204-S039 & AmpR \\
\hline V1378 & pAAV-SOX2-OCT4-cRE-MLP-E01211-S041 & AmpR \\
\hline V1378 & pAAV-SOX2-OCT4-CRE-MLP-E01212-S041 & AmpR \\
\hline V1378 & pAAV-SOX2-OCT4-cRE-MLP-E01213-S041 & AmpR \\
\hline V1377 & pAAV-SOX2-RE-MLP-E01205-S040 & AmpR \\
\hline V1377 & pAAV-SOX2-RE-MLP-E01206-S040 & AmpR \\
\hline V1377 & PAAV-SOX2-RE-MLP-E01207-S040 & AmpR \\
\hline V1379 & pAAV-SP1-RE-v1-MLP-E01216-S042 & AmpR \\
\hline V1379 & pAAV-SP1-RE-v1-MLP-E01337-S042 & AmpR \\
\hline V1379 & pAAV-SP1-RE-v1-MLP-E01339-S042 & AmpR \\
\hline V1380 & pAAV-SP1-RE-v2-MLP-E01217-S043 & AmpR \\
\hline V1380 & pAAV-SP1-RE-v2-MLP-E01218-S043 & AmpR \\
\hline V1380 & PAAV-SP1-RE-V2-MLP-E01219-S043 & AmpR \\
\hline V1385 & pAAV-SRE-MLP-E01242-S048 & AmpR \\
\hline V1385 & pAAV-SRE-MLP-E01243-S048 & AmpR \\
\hline V1385 & PAAV-SRE-MLP-E01245-S048 & AmpR \\
\hline V1381 & pAAV-SREBP-RE-v1-MLP-E01221-S044 & AmpR \\
\hline V1381 & pAAV-SREBP-RE-v1-MLP-E01222-S044 & AmpR \\
\hline V1381 & pAAV-SREBP-RE-v1-MLP-E01223-S044 & AmpR \\
\hline V1382 & pAAV-SREBP-RE-v2-MLP-E01226-S045 & AmpR \\
\hline V1382 & pAAV-SREBP-RE-v2-MLP-E01227-S045 & AmpR \\
\hline V1382 & pAAV-SREBP-RE-v2-MLP-E01228-S045 & AmpR \\
\hline V1383 & pAAV-SREBP-RE-v3-MLP-E01233-S046 & AmpR \\
\hline V1383 & pAAV-SREBP-RE-v3-MLP-E01234-S046 & AmpR \\
\hline V1383 & PAAV-SREBP-RE-v3-MLP-E01235-S046 & AmpR \\
\hline V1384 & pAAV-SRF-RE-v2-MLP-E01237-S047 & AmpR \\
\hline
\end{tabular}




\begin{tabular}{|c|c|c|}
\hline V1384 & pAAV-SRF-RE-v2-MLP-E01239-S047 & AmpR \\
\hline V1384 & pAAV-SRF-RE-v2-MLP-E01240-S047 & AmpR \\
\hline V1386 & pAAV-STAT-RE-v1-MLP-E01246-S049 & AmpR \\
\hline V1386 & pAAV-STAT-RE-v1-MLP-E01248-S049 & AmpR \\
\hline V1386 & pAAV-STAT-RE-v1-MLP-E01249-S049 & AmpR \\
\hline V1390 & pAAV-TEAD-RE-v3-MLP-E01262-S053 & AmpR \\
\hline V1390 & pAAV-TEAD-RE-v3-MLP-E01263-S053 & AmpR \\
\hline V1390 & pAAV-TEAD-RE-V3-MLP-E01264-S053 & AmpR \\
\hline V1474 & pAAV-TEAD-RE-v4-MLP-E01356-S110 & AmpR \\
\hline V1474 & pAAV-TEAD-RE-v4-MLP-E01357-S110 & AmpR \\
\hline V1474 & pAAV-TEAD-RE-v4-MLP-E01358-S110 & AmpR \\
\hline V1228 & pAAV-TEADrev-RE-MLP-E00569-S070 & AmpR \\
\hline V1228 & pAAV-TEADrev-RE-MLP-E00575-S070 & AmpR \\
\hline V1228 & pAAV-TEADrev-RE-MLP-E00579-S070 & AmpR \\
\hline V1391 & pAAV-UPRE-v1-MLP-E01265-S054 & AmpR \\
\hline V1391 & pAAV-UPRE-v1-MLP-E01266-S054 & AmpR \\
\hline V1391 & pAAV-UPRE-v1-MLP-E01267-S054 & AmpR \\
\hline V1392 & pAAV-UPRE-v2-MLP-E01270-S055 & AmpR \\
\hline V1392 & pAAV-UPRE-v2-MLP-E01272-S055 & AmpR \\
\hline V1392 & pAAV-UPRE-v2-MLP-E01273-S055 & AmpR \\
\hline V1387 & pAAV-Wnt-RE-v2-MLP-E01250-S051 & AmpR \\
\hline V1387 & pAAV-Wnt-RE-v2-MLP-E01251-S051 & AmpR \\
\hline V1387 & pAAV-Wnt-RE-v2-MLP-E01252-S051 & AmpR \\
\hline V1393 & pAAV-YY1-RE-v1-MLP-E01276-S056 & AmpR \\
\hline V1393 & pAAV-YY1-RE-v1-MLP-E01277-S056 & AmpR \\
\hline V1393 & pAAV-YY1-RE-v1-MLP-E01279-S056 & AmpR \\
\hline V1394 & pAAV-YY1-RE-v2-MLP-E01280-S057 & AmpR \\
\hline V1394 & pAAV-YY1-RE-v2-MLP-E01281-S057 & AmpR \\
\hline V1394 & pAAV-YY1-RE-v2-MLP-E01282-S057 & AmpR \\
\hline \multicolumn{3}{|c|}{ Promoter-EXT reporters } \\
\hline V1413 & pAAV-sEGFP-CXCL1-E00188-S086 & AmpR \\
\hline V1413 & pAAV-sEGFP-CXCL1-E00189-S086 & AmpR \\
\hline V1413 & pAAV-sEGFP-CXCL1-E00190-S086 & AmpR \\
\hline V1418 & pAAV-sEGFP-DUSP1-E00199-S091 & AmpR \\
\hline V1418 & pAAV-sEGFP-DUSP1-E00200-S091 & AmpR \\
\hline V1418 & pAAV-sEGFP-DUSP1-E00201-S091 & AmpR \\
\hline V1419 & pAAV-sEGFP-DUSP5-E01319-S092 & AmpR \\
\hline V1419 & pAAV-sEGFP-DUSP5-E01320-S092 & AmpR \\
\hline V1419 & pAAV-sEGFP-DUSP5-E01321-S092 & AmpR \\
\hline V1420 & pAAV-sEGFP-EGR1-E00209-S093 & AmpR \\
\hline V1420 & pAAV-sEGFP-EGR1-E00210-S093 & AmpR \\
\hline V1420 & pAAV-sEGFP-EGR1-E00212-S093 & AmpR \\
\hline V1421 & pAAV-sEGFP-EGR2-E00114-S094 & AmpR \\
\hline V1421 & pAAV-sEGFP-EGR2-E00115-S094 & AmpR \\
\hline V1421 & pAAV-sEGFP-EGR2-E00116-S094 & AmpR \\
\hline
\end{tabular}




\begin{tabular}{|c|c|c|}
\hline V1405 & pAAV-sEGFP-EIF2AK2-E00144-S078 & AmpR \\
\hline V1405 & pAAV-sEGFP-EIF2AK2-E00145-S078 & AmpR \\
\hline V1405 & pAAV-sEGFP-EIF2AK2-E00146-S078 & AmpR \\
\hline V1422 & pAAV-sEGFP-FOS-E00120-S095 & AmpR \\
\hline V1422 & pAAV-sEGFP-FOS-E00121-S095 & AmpR \\
\hline V1411 & pAAV-sEGFP-FOSB-E00174-S084 & AmpR \\
\hline V1411 & pAAV-sEGFP-FOSB-E00175-S084 & AmpR \\
\hline V1415 & pAAV-sEGFP-hDLG4-1-E00221-S088 & AmpR \\
\hline V1415 & pAAV-sEGFP-hDLG4-1-E00224-S088 & AmpR \\
\hline V1416 & pAAV-sEGFP-hDLG4-2-E00228-S089 & AmpR \\
\hline V1416 & pAAV-sEGFP-hDLG4-2-E00230-S089 & AmpR \\
\hline V1404 & pAAV-sEGFP-HSPA1A-E00139-S077 & AmpR \\
\hline V1404 & pAAV-sEGFP-HSPA1A-E00140-S077 & AmpR \\
\hline V1423 & pAAV-sEGFP-IL6-E00219-S096 & AmpR \\
\hline V1423 & pAAV-sEGFP-IL6-E01348-S096 & AmpR \\
\hline V1423 & pAAV-sEGFP-IL6-E01365-S096 & AmpR \\
\hline V1407 & pAAV-sEGFP-IL8-E00109-S080 & AmpR \\
\hline V1407 & pAAV-sEGFP-IL8-E00110-S080 & AmpR \\
\hline V1407 & pAAV-sEGFP-IL8-E01364-S080 & AmpR \\
\hline V1424 & pAAV-sEGFP-JUN-E00562-S097 & AmpR \\
\hline V1424 & pAAV-sEGFP-JUN-E00563-S097 & AmpR \\
\hline V1417 & pAAV-sEGFP-mDlg4-2-E00239-S090 & AmpR \\
\hline V1417 & pAAV-sEGFP-mDlg4-2-E00240-S090 & AmpR \\
\hline V1408 & pAAV-sEGFP-NFKB2-E00156-S081 & AmpR \\
\hline V1408 & pAAV-sEGFP-NFKB2-E01345-S081 & AmpR \\
\hline V1408 & pAAV-sEGFP-NFKB2-E01347-S081 & AmpR \\
\hline V1444 & pAAV-sEGFP-Npas4-E01354-S098 & AmpR \\
\hline V1412 & pAAV-sEGFP-NR4A1-E00180-S085 & AmpR \\
\hline V1412 & pAAV-sEGFP-NR4A1-E00185-S085 & AmpR \\
\hline V1402 & pAAV-sEGFP-PPP1R15A-E00127-S075 & AmpR \\
\hline V1402 & pAAV-sEGFP-PPP1R15A-E00128-S075 & AmpR \\
\hline V1406 & pAAV-sEGFP-PSMB9-E00150-S079 & AmpR \\
\hline V1406 & pAAV-sEGFP-PSMB9-E00151-S079 & AmpR \\
\hline V1406 & pAAV-sEGFP-PSMB9-E00152-S079 & AmpR \\
\hline V1414 & pAAV-sEGFP-sDLG4-E00193-S087 & AmpR \\
\hline V1414 & pAAV-sEGFP-sDLG4-E00195-S087 & AmpR \\
\hline V1403 & pAAV-sEGFP-TNFA-E00133-S076 & AmpR \\
\hline V1403 & pAAV-sEGFP-TNFA-E00135-S076 & AmpR \\
\hline V1409 & pAAV-sEGFP-TP53INP1-E00160-S082 & AmpR \\
\hline V1409 & pAAV-sEGFP-TP53INP1-E00163-S082 & AmpR \\
\hline V1410 & pAAV-sEGFP-TP73-E00165-S083 & AmpR \\
\hline V1410 & pAAV-sEGFP-TP73-E00166-S083 & AmpR \\
\hline V1410 & pAAV-sEGFP-TP73-E00167-S083 & AmpR \\
\hline
\end{tabular}




\section{Methods}

\subsection{Molecular biology methods}

\subsubsection{Polymerase chain reaction}

The polymerase chain reaction (PCR) is a widely used method for the amplification of DNA fragments in vitro. Thermo stable polymerases like the Taq-polymerase of the bacteria Thermus aquaticus or the Pfu-polymerase of the archaea Pyrococcus furiosus catalyse the reaction. Because the DNA-synthesis cannot start from de novo two primer flanking the sequence to amplify, a sense primer for the plus-strand and an antisense primer for the minus-strand, are needed. The PCR happens in the following steps.

Denaturation: The initial denaturation at $95^{\circ} \mathrm{C}$ for 2-3 min, 5-10 min for HotStarTag Plus DNA polymerase from Qiagen, leads to the break of secondary structures and the separation of the DNA strands. The initial denaturation is followed by a further denaturation for 30-45 sec that is the first step of every cycle during the PCR.

Annealing: In this step the primer anneal to the complementary DNA strands. It happens for $20-30 \mathrm{sec}$ at a temperature $2-4^{\circ} \mathrm{C}$ below the melting temperature of the primer.

Elongation: During the elongation at a temperature of $72^{\circ} \mathrm{C}$ the synthesis of the new DNA strands occurs. It takes about $1 \mathrm{~min} / 1 \mathrm{~kb}$ DNA length depending on the synthesis rate of the polymerase. After the elongation a new cycle starts with the denaturation of the new synthesised DNA. Depending on the DNA template (e.g. cDNA, genomic DNA or plasmid) 15-35 cycles were used. The last cycle follows an additional elongation step for 5-10 min to ensure a complete elongation. In the end the sample was kept at $10^{\circ} \mathrm{C}$ until further processes.

Scheme of a typical $20 \mu$ I PCR sample:

$$
\begin{array}{rl}
X \mu l & \text { DNA template }(10-100 \mathrm{ng}) \\
2 \mu \mathrm{l} & 10 x \text { buffer }\left(\text { with } \mathrm{MgCl}_{2}\right) \\
2 \mu \mathrm{l} & \text { dNTPs }(0.2 \mathrm{mM}) \\
1 \mu \mathrm{l} & \text { sense primer }(10 \mathrm{pmol} / \mu \mathrm{l}) \\
1 \mu \mathrm{l} & \text { antisense primer }(10 \mathrm{pmol} / \mu \mathrm{l}) \\
0.5 \mu \mathrm{l} & \text { polymerase }(5 \mathrm{U} / \mu \mathrm{l}) \\
\mathrm{X} \mu \mathrm{l} & \mathrm{H}_{2} \mathrm{O}
\end{array}
$$




\subsubsection{Agarose gel electrophoresis}

For the separation of DNA fragments up to $8 \mathrm{~kb} 1-3 \%$ agarose gels were used. Therefore the agarose was dissolved in 1XTAE buffer by heating up in a microwave. The agarose was then poured either directly in gel-casting form or at first cooled down to ca. $50^{\circ} \mathrm{C}$ and supplemented with ethidium bromide to a final concentration of $1 \mu \mathrm{g} / \mathrm{ml}$. Pockets for sample loading were obtained by inserting combs into the liquid agarose. After polymerisation the agarose were storable at $4^{\circ} \mathrm{C}$ for up to 4 weeks. For DNA separation the gel was placed in a running chamber containing 1xTAE buffer. The DNA sample was supplemented with either $6 \mathrm{x}$ Loading dye from Thermo Fisher Scientific for ethidium bromide gels or 10x GelRed/Orange $\mathrm{G}$ loading buffer for ethidium bromide free gels and loaded on the gel. The applied voltage was between 90-180 V, dependent on the size of the running chamber. The DNA intercalated with either ethidium bromide or GelRed was visualised by UV light (260-300nm). For size quantification the $50 \mathrm{bp}, 100 \mathrm{bp}$ or $1 \mathrm{~kb}$ ladder from Thermo Fisher Scientific was used. The casting form, combs and running chambers were made by the workshop of the Max-Planck-Institute for Experimental Medicine.

\subsubsection{Restriction digest of DNA}

For restriction digest of DNA restriction endonucleases type II were used. They recognise and cleave within palindromic sequences of four, six or eight bases and produce either $5^{\prime}-/ 3^{\prime}$ - overhangs (sticky ends) or blunt ends. For the reaction one of four standard 10x buffers was used according to the manufacture's protocol (New England Biolabs, NEB). For diagnostic digests 0.5-1 $\mu \mathrm{g}$ DNA, for preparative digests 5-20 $\mu \mathrm{g}$ DNA were used in a reaction volume of 30-60 $\mu \mathrm{l}$. The reactions were usually incubated at $37^{\circ} \mathrm{C}$ for at least 30 min up to overnight, dependent on the DNA amount.

\subsubsection{Classical molecular cloning}

PCR products were generates using HotStarTaq Plus DNA polymerase from Qiagen, which produces a single 3'- adenosine overhangs. The PCR product was then cloned into the pGEM-T vector (Promega) by ligation with T4 DNA ligase (Promega) according to the manufacture's protocol. The pGEM-T vector is provided in a linearized form with 
single 3'-thymidine overhangs. The ligation was transformed into bacteria and plated on LB-Amp-IPTG-X-Gal agar plates for blue white screening over night at $37^{\circ} \mathrm{C}$. Clones from white colonies were sequenced. 5-10 $\mu \mathrm{g}$ of the PGEM-T insert was cut with the same or compatible restriction enzymes like 5-20 $\mu \mathrm{g}$ of the vector of interest in a volume of $30-60 \mu \mathrm{l}$ with $5-10$ units of the restriction enzymes. The temperature is dependent on the enzymes, usually at $37^{\circ} \mathrm{C}$. Afterwards the vector sample was incubated with $1 \mu \mathrm{l}$ alkaline phosphatase (CIP from NEB) for $20 \mathrm{~min}$ at $37^{\circ} \mathrm{C}$. The DNA fragments were analysed on a $1.5 \%$ agarose gel and the bands of the desired size were cut out and purified with help of the gel and PCR clean up kit from Macherey \& Nagel. The purified Fragments were ligated in a molar vector: insert ratio of 1:3 using $0.5 \mu \mathrm{l}$ T4 ligase in a total volume of $20 \mu \mathrm{l}$ for first 2-3 $\mathrm{h}$ at room temperature and then over night at $4^{\circ} \mathrm{C}$. Afterwards the reaction was transformed into OneShot Mach1 or MegaX DH10B cells.

\subsubsection{One-way Gateway cloning}

The Gateway recombination technology is based on the sequence specific recombination of the bacteriophage $\lambda$ to recombine DNA in a host genome. The sitespecific recombination requires specific sequences (attachment sites, att-sites) flanking the sequence of interest and combinatory att-sites on the target DNA (Hartley et al., 2000). The recombination process is catalysed by bacteriophage $\lambda$ enzymes Integrase (Int), Integration Host Factor (IHF) and Excisionase (Xis). The benefit of this method is the fast and efficient recombination of an ORF of interest into different expression vectors without using restriction enzymes, fragment purification and ligation. One feature of the gateway technology is that only bacteria carrying successfully recombined vectors can survive as not recombined vectors contain the ccdB-gene, a toxic gyrase inhibitor. The process of cloning an ORF of interest into an expression vector is divided into two steps.

Creating an entry vector: Specific recombination sites (attB1 and attB2) have to be added to both ends of the sequence of interest by PCR. In a BP reaction the attB sites recombine with recognition sites (attP1 and attP2) on a donor vector (pDONR, Thermo Fisher Scientific). The recombination is catalysed by the BP clonase II enzyme mix. 
Successfully recombined clones represent entry clones (pENTR) with attL1 and attL2 sites (product of the recombination of attB and attP sites).

A BP reaction consists of:

$3 \mu \mathrm{l} \quad$ attB1-attB2 PCR

$1 \mu \mathrm{l} \quad$ DONR (100 ng)

$1 \mu \mathrm{l} \quad$ BP clonase II mix

The reaction was incubated over night at room temperature. For deactivating of enzymes $1 \mu \mathrm{l}$ proteinase $\mathrm{K}$ was added and incubated for $10 \mathrm{~min}$ at $37^{\circ} \mathrm{C}$. 1-5 $\mu \mathrm{l}$ of the sample was used for transformation in chemically or electro competent cells. Entry clones were selected on agar plates with the appropriate antibiotic and sequenced.

Creating an expression vector: To generate the final expression vector, the entry vector with attL1 and attL2 sites is recombined in a $L R$ reaction with a destination vector (pDEST) carrying the recognition sites attR1 and attR2 and the $\operatorname{ccdB}$ gene. The recombination is catalysed by a LR clonase II enzyme mix.

A LR reaction consists of:

$\begin{aligned} 1 \mu \mathrm{l} & \text { pENTR (50-200 ng) } \\ 1 \mu \mathrm{l} & \text { pDEST (100 ng) } \\ 0.5 \mu \mathrm{l} & \text { LR clonase II mix }\end{aligned}$

The reaction was incubated for 3-4 $\mathrm{h}$ or overnight at room temperature and deactivated by adding $1 \mathrm{ml}$ proteinase $\mathrm{K}$ and incubating for $10 \mathrm{~min}$ at $37^{\circ} \mathrm{C} .1 .5 \mu \mathrm{l}$ of the LR reaction was used for transformation into chemically or electro competent cells.

\subsubsection{Multisite Gateway cloning}

The Multisite Gateway technology is an efficient and simple method to assemble up to 4 DNA fragments into one expression vector using multiple specific recombination sites. The recombination of 3 fragments was applied to generate reporter constructs. Detailed information can be found in the Multisite Gateway Pro user manual from Thermo Fisher Scientific. The principle of the Multisite Gateway cloning is shown in Figure 5. It is based on the specific recombination of different att-sites. Compared to the one-way Gateway method in the Multisite Gateway system the entry clone containing the middle of three DNA fragments carries attR sites, which recombine in a $L R$ reaction with the attL sites of entry clones containing the two flanking DNA 
fragments. In the same time the two outer attL sites (attB1 and attB2) of the first and third entry clone recombine with the attR recognition sites of the destination vector.

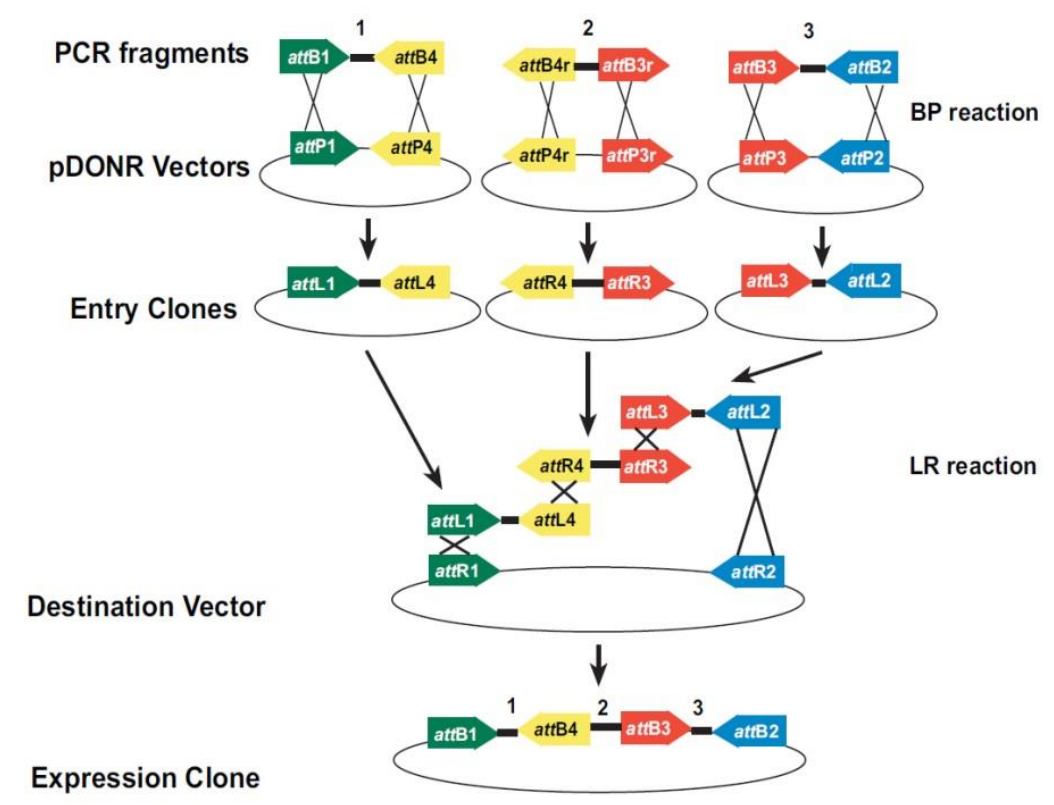

Figure 5: Scheme of the three fragments assembly using Multisite Gateway Technology.

Three DNA fragments can be cloned simultaneously into one vector using different recombination sites. The picture is adapted from the Multisite Gateway Pro user manual.

Three PB reactions recombine PCR products with the corresponding pDONR vectors:

B1-B4 PCR product + pDONR (P1-P4) $\rightarrow$ pENTR (L1-L4)

B4r-B3r PCR product + pDONR (P4r-P3r) $\rightarrow$ pENTR (R4-R3)

B3-B2 PCR product + pDONR $($ P3-P2) $\rightarrow$ pENTR (L3-L2)

$A B P$ reaction consists of:

$3 \mu \mathrm{l} \quad$ PCR product

$1 \mu \mathrm{l} \quad$ pDONR (100 ng)

$1 \mu \mathrm{l} \quad$ BP clonase II mix

The reaction was incubated over night at room temperature with an additional proteinase $\mathrm{K}$ deactivation for $10 \mathrm{~min}$ at $37^{\circ} \mathrm{C}$ and transformation into One Shot Mach1 cells. The purified vector plasmids were sequenced. For the multisite LR reaction $10 \mathrm{fmol}$ of each entry vector and $20 \mathrm{fmol}$ of the destination vector was used. The following formula was applied to calculate the amount of each vector, with $\mathrm{N}$ being the number of bases of the vector.

$$
n=(f m o l)(N)\left(\frac{660 f g}{f m o l}\right)\left(\frac{1 n g}{10^{6} f g}\right)
$$


The vectors were diluted to the corresponding concentrations $(\mathrm{ng} / \mu \mathrm{l})$. The multisite $L R$ was catalysed by the LR clonase II Plus enzyme mix.

A multisite LR reaction consists of:

$\begin{array}{ll}1 \mu \mathrm{l} & \text { pENTR (L1-L4, 10fmol) } \\ 1 \mu \mathrm{l} & \text { pENTR (R4-R3, 10fmol) } \\ 1 \mu \mathrm{l} & \text { pENTR (L3-L2, 10fmol) } \\ 1 \mu \mathrm{l} & \text { pDEST (20fmol) } \\ 1 \mu \mathrm{l} & \text { LR clonase II Plus mix }\end{array}$

The reaction was incubated over night at room temperature, deactivated with $1 \mu \mathrm{l}$ proteinase $\mathrm{K}$ for $10 \mathrm{~min}$ at $37^{\circ} \mathrm{C}$ and subsequently transformed into One Shot Mach1 cells. The expression vectors were finally sequenced.

\subsubsection{Photometric concentration determination of nuclear acids}

According to the Lambert-Beer law the concentration of a solution is direct proportional to their extinction, $A=\varepsilon^{*} c^{*} \lambda$, with $\varepsilon$ being the molar extinction coefficient (unit: $\mathrm{M}^{-1} \mathrm{~cm}^{-1}$ ), c the concentration (unit: $M$ ) and $\lambda$ the optic path length (cuvette thickness). The extinction coefficients for nuclear acids are at $\lambda=260 \mathrm{~nm}$ :

Cytosine: $\varepsilon=7050 \mathrm{M}^{-1} \mathrm{~cm}^{-1}$

Guanine: $\varepsilon=12010 \mathrm{M}^{-1} \mathrm{~cm}^{-1}$

Adenine: $\varepsilon=15200 \mathrm{M}^{-1} \mathrm{~cm}^{-1}$

Thymine: $\varepsilon=8400 \mathrm{M}^{-1} \mathrm{~cm}^{-1} \quad$ (Uracil: $\varepsilon=8111 \mathrm{M}^{-1} \mathrm{~cm}^{-1}$ )

For the determination of nuclear acid concentration the following relation between measured absorption and concentration was used:

$1 \mathrm{OD}_{260 \mathrm{~nm}}=50 \mathrm{mg} / \mathrm{ml}$ for dsDNA

$1 \mathrm{OD}_{260 \mathrm{~nm}}=40 \mathrm{mg} / \mathrm{ml}$ for ssRNA

$1 \mathrm{OD}_{260 \mathrm{~nm}}=33 \mathrm{mg} / \mathrm{ml}$ for ssOligonucleotides

The formula for dsDNA concentration was:

Concentration $[\mu \mathrm{g} / \mu \mathrm{l}]=\mathrm{OD}_{260} \times 50 \times$ dilution factor $/ 1000$.

To determine the purity of the nuclear acid solution the relation of the absorption at $260 \mathrm{~nm}$ and $280 \mathrm{~nm}$ was calculated. Clear samples of DNA and RNA should give a value of 1.8 to 2.0 . 
The measurements were carried out by a biophotometer (Eppendorf) or Picodrop spectrophotometer (Picodrop Limited). For the biophotometer a dilution of 1:100 in a volume of $100 \mu \mathrm{l}$ was used.

\subsubsection{Fluorometric concentration determination of nuclear acids}

To quantify the concentration of final samples for Next Generation Sequencing a more accurate and precise method to determine low nuclear acid concentrations than measuring the UV absorbance at $260 \mathrm{~nm}$ was needed. This was achieved by using the Qubit 2.0 Fluorometer (Invitrogen). Fluorescence based dyes bind specifically to DNA, RNA or protein and emit signal only when coupled to the target molecules. Thereby only the desired type of nuclear acid is measured. With the Qubit dsDNA High Sensitivity (HS) Assay Kit DNA concentrations in the range of $10 \mathrm{pg} / \mu \mathrm{l}$ to $100 \mathrm{ng} / \mu \mathrm{l} \mathrm{can}$ be determined. $5 \mu \mathrm{l}$ of the DNA sample and $10 \mu \mathrm{l}$ of two DNA standards were quantified according to the manufacture's protocol.

\subsubsection{Transformation of chemically competent bacteria}

20-50 $\mu$ l of chemically competent bacteria were thawed on ice. After adding 1-5 $\mu \mathrm{l}$ of a recombination or ligation cells were left on ice for additional $20 \mathrm{~min}$ followed by a heat shock for $42 \mathrm{sec}$ at $42^{\circ} \mathrm{C}$. For recovery and expression of resistance genes $800 \mu \mathrm{l}$ of prewarmed SOC medium was added and cells were incubated for $45 \mathrm{~min}$ at $37^{\circ} \mathrm{C}$ and $160 \mathrm{rpm}$ shaking. After this cells were centrifuged at for $2 \mathrm{~min}$ at $11000 \mathrm{rpm}$ and the cell pellet resuspended in $100 \mu \mathrm{l}$ SOC medium and plated on an agar plate supplemented with the appropriate antibiotic. The plates were incubated over night at $37^{\circ} \mathrm{C}$

\subsubsection{Transformation of electro competent bacteria}

$5 \mu \mathrm{l}$ of electro competent bacteria were diluted $1: 10$ in a $10 \%$ glycerol solution and mixed with 1-3 $\mu$ l of a recombination or ligation. The plasmid/DNA mixture was transferred in an on ice pre cooled electroporation cuvette $(1 \mathrm{~mm})$ and electroporated at $1700 \mathrm{kV}, 25 \mu \mathrm{F}$ and $200 \mathrm{ohm}$. Afterwards the cells were transferred into $800 \mu \mathrm{l} \mathrm{SOC}$ medium and incubated for $45 \mathrm{~min}$ at $37^{\circ} \mathrm{C}$ and $160 \mathrm{rpm}$ shaking. Then the cells were 
centrifuged for $2 \mathrm{~min}$ at $11000 \mathrm{rpm}$, the pellet resuspended in $100 \mu \mathrm{l} \mathrm{SOC}$ medium and plates on an agar plate with the appropriate antibiotic. The plates were incubated over night at $37^{\circ} \mathrm{C}$.

\subsubsection{Isolation of DNA}

Plasmid DNA was prepared from fresh overnight LB cultures. For small plasmid amounts 2-4ml (Mini-prep) and for larger plasmid amounts 100ml (Midi-prep) were used. The preparation occurred with help of the corresponding kits from Macherey $\&$ Nagel using the manufacture's protocols. The purified plasmid DNA was always eluted in $100 \mu \mathrm{l}$ of either TE buffer or in case of sequencing the plasmid DNA in double deionised water.

\subsubsection{DNA sequencing}

The plasmid DNA sequencing was done by the institutes' core facility AGCTlab (department of neurobiology) of the MPI of Experimental Medicine in Göttingen and from February 2014 by the sequencing service of the faculty of biology of the LudwigMaximilians-University Munich. The procedure based on a modified dideoxy chain termination method according to Sanger (Sanger et al., 1977).

\subsection{Cell biology methods}

\subsubsection{Coating of cell culture plates}

For an improved adhesion of cells at the plate surface all multi-well plates (96-well, 12-well, 24-well, 6-well) and for PC12 cells also the culture dishes required a coating with Poly-L-Lysine (PLL). Plates were incubated with $200 \mu \mathrm{g} / \mathrm{ml} \mathrm{PLL}$ solution for $20 \mathrm{~min}$ at room temperature, washed three times with water and dried under a flow hood.

\subsubsection{Culturing and passaging of eukaryotic cells}

The eukaryotic cell lines were incubated in $15 \mathrm{~cm}$ dishes (Falcon) at $37^{\circ} \mathrm{C}$ with $5 \% \mathrm{CO}_{2}$ in a cell culture incubator. After reaching $80-90 \%$ confluence the cells were split. For 
this the medium was removed and the cells washed twice with 1xPBS. To remove cells from dish the surface was covered with trypsin and incubated for $2-5 \min$ at $37^{\circ} \mathrm{C}$. Adding growth medium containing FBS stopped the reaction. The cells were collected in a falcon tube and centrifuged for $3 \mathrm{~min}$ at $800 \mathrm{rpm}$. The supernatant was discarded, the pellet resuspended and a volume of $1 / 4$ to $1 / 10$ plated on a new dish. PC12 cells were before plating triturated 3-5 times through a $24 \mathrm{G}(0.55 \mathrm{~mm})$ needle using a $10 \mathrm{ml}$ syringe and plated on PLL coated dishes.

\subsubsection{Freezing of mammalian cells}

Before handling the cells $2 \mathrm{ml}$ cryotubes were labelled and filled with $500 \mu \mathrm{l}$ of ice-cold 2xFreezing medium. Cryotubes were stored on ice. Cells were frozen at $80-90 \%$ confluence. They were removed from the dish by trypsination and centrifuged for $3 \mathrm{~min}$ at $800 \mathrm{rpm}$. The pellet was resuspended in growth medium at a concentration of $6-10 \times 10^{6}$ cells $/ \mathrm{ml}$ medium. $500 \mu \mathrm{l}$ of cell suspension was transferred in the prepared cryotubes, mixed gently by inverting and stored in an isopropyl alcohol filled Nalgene freezing container "Mr. Frosty" at $-80^{\circ} \mathrm{C}$ for one day. The Nalgene freezing container is a system designed to cool down approximately $1^{\circ} \mathrm{C} / \mathrm{min}$. Afterwards the tubes were stored in liquid nitrogen $\left(-196^{\circ} \mathrm{C}\right)$ for long term storage.

\subsubsection{Thawing of mammalian cells}

Cryotubes with frozen cells were taken out of liquid nitrogen and thawed quickly in a $37^{\circ} \mathrm{C}$ warm water bath. After thawing the cells were mixed with $2 \mathrm{ml} 37^{\circ} \mathrm{C}$ warm growth medium and centrifuged for $5 \mathrm{~min}$ at $800 \mathrm{rpm}$. The supernatant was discarded and the pellet resuspended and plated on a $15 \mathrm{~cm}$ cell culture dish with fresh growth medium. 


\subsubsection{Transfection of eukaryotic cells}

All eukaryotic cell lines were transfected using Lipofectamine 2000 (Thermo Fisher Scientific). The cell number was dependent of the cell line and the plate format. The used cell numbers are indicated below:

\begin{tabular}{lcccc} 
Cell line & 6-well & 12-well & 24-well & 96-well \\
\hline PC12 & 1.000 .000 & 400.000 & 200.000 & 40.000 \\
\hline U2OS & 500.000 & 200.000 & 100.000 & 15.000 \\
\hline CHO & 400.000 & - & - & 10.000 \\
\hline
\end{tabular}

\section{On-plate transfection:}

The manufacture's protocol was modified to improve transfection efficiency. For 96-well plates 30-50 ng plasmid DNA, $0.2 \mu$ l Lipofectamine and $30 \mu$ optiMEM was used. For larger well sizes the amount of plasmid and Lipofectamine was increased by the factor of increased well surface. Cells were plated one day before transfection. For transfection the prepared plasmid mixes and the Lipofectamine was diluted each in half of the volume of optiMEM and incubated for $5 \mathrm{~min}$ at room temperature. Both solutions were mix and incubated for further $20 \mathrm{~min}$ at room temperature. The medium was completely removed and the Lipofectamine/DNA mixture was added. After $2 \mathrm{~h}$ for 96 -well plates $60 \mu \mathrm{l}$ of growth medium was added. For larger multi-well plates the transfection solution was completely exchanges by growth medium.

In-solution transfection:

For in-solution transfections the followed conditions or equal ratios were used.

\begin{tabular}{cccccc} 
DNA & OptiMEM & Lipofectamine & $\begin{array}{c}\text { Cell } \\
\text { number }\end{array}$ & $\begin{array}{c}\text { Cell } \\
\text { volume }\end{array}$ & $\begin{array}{c}\text { Total } \\
\text { volume }\end{array}$ \\
\hline $6 \mu \mathrm{g}$ & $2 \times 250 \mu \mathrm{l}$ & $20 \mu \mathrm{l}$ & 6.000 .000 & $2 \mathrm{ml}$ & $2,5 \mathrm{ml}$ \\
\hline $1,2 \mu \mathrm{g}$ & $2 \times 50 \mu \mathrm{l}$ & $4 \mu \mathrm{l}$ & 1.200 .000 & $400 \mu \mathrm{l}$ & $500 \mu \mathrm{l}$ \\
\hline $1 \mu \mathrm{g}$ & $2 \times 40 \mu \mathrm{l}$ & $3,3 \mu \mathrm{l}$ & 1.000 .000 & $400 \mu \mathrm{l}$ & $500 \mu \mathrm{l}$ \\
\hline
\end{tabular}

The transfection was done in $5 \mathrm{ml}$ cryotubes. The prepared plasmid mixes and the Lipofectamine was diluted each in half of the volume of optiMEM and incubated for $5 \mathrm{~min}$ at room temperature. Both solutions were mix and incubated for further $20 \mathrm{~min}$ 
at room temperature. Cells resuspended in the appropriate volume and added to the transfection mix. The solution was inverted three times and incubated lying flat in the incubator for $2 \mathrm{~h}$. Before plating the cell-transfection suspension was centrifuged and resuspended in maintenance medium.

\subsubsection{Luciferase reporter gene assays}

The principle of the luciferase reporter gene assays is based on the activated gene expression of the Firefly luciferase enzyme. The luciferase enzyme catalyses the oxidation of luciferin and emit light of a defined wavelength.

\section{Luciferase assays using a plate reader:}

Beside the Firefly luciferase (FF-luciferase) the reporter gene for the Renilla luciferase (RN-luciferase) was cotransfected to control the cell number, transfection efficiency and health status of the cells. The RN-luciferase was expressed under the control of the constitutively active thymidine kinase promoter (TK-promoter). The assays were performed in 96-well white plates with 6 wells as replicates per condition. After transfection with lipofectamine cells were incubated dependent on the assay for $24-48 \mathrm{~h}$ at $37^{\circ} \mathrm{C}$ and $5 \% \mathrm{CO}_{2}$. After the experiment the medium was removed and the cells were lysed with $30 \mu \mathrm{l}$ passive lysis buffer (Promega). The lysates were measured either immediately after incubated for $20 \mathrm{~min}$ on a tumbling table at $50 \mathrm{rpm}$ or frozen at $-20^{\circ} \mathrm{C}$. The reading of both FF- and RN-luciferase was carried out with a Mithras LB 940 Microplate Reader (Berthold Technologies) and the software MicroWin2000 under the following settings:

$55 \mu \mathrm{l}$ Firefly luciferase assay buffer

5 min delay

$2 \mathrm{sec}$ measurement of light

$55 \mu \mathrm{l}$ Renilla luciferase assay buffer

$2 \mathrm{sec}$ measurement of light

The data was exported from MicroWin2000 to Excel and the means of the 6 replicates for the Firefly and the Renilla readings were calculated. 
Luciferase assays using a 32-channel luminometer (lumiCycle 32 by ActiMetrics):

The lumicycler performs live monitoring of the FF-luciferase activity over the complete time of the experiment. 32 samples, each in a $3.5 \mathrm{~cm}$ dish, can be measured the same time. The transfections were done in $3.5 \mathrm{~cm}$ dishes. For measuring the FF-luciferase activity the medium from the transfected cells was exchanged by the appropriate medium supplemented with 0,1\% luciferin (Promega), the dishes were wrapped with parafilm and transferred to the lumicycler, which is located inside a cell culture incubator at $37^{\circ} \mathrm{C}$ and $5 \% \mathrm{CO}_{2}$.

\subsubsection{Dose response assays}

Luciferase based dose dependent GPCR activations were measured in 96-well plates with 6 wells per condition as replicates. The compounds were step wise diluted in assay medium from a stock concentration to final concentrations in a range from $10^{-4}$ to $10^{-11} \mathrm{M}$ in logarithmic and semi logarithmic steps. Each dilution results from the next higher concentration and serves for the next lower concentration. EXT based dose responses were carried out in 6-well plated in logarithmic steps.

Dose response data from firefly luciferase as well as from EXT reads were first calculated with Excel by converting the means of the replicates from either firefly or EXT reads into percentage of activity. Thereby the lowest value was set to 0 by subtracting the lowest mean from all replicates. From the resultant means the highest was set to $100 \%$. Beside the means the standard error of the mean (SEM) of all replicates was calculated.

Dose response curves and $\mathrm{EC}_{50}$ and $\mathrm{IC}_{50}$ values respectively were generated using the dose response curve $(\mathrm{drc})$ package for $\mathrm{R}$. 


\subsection{Preparation of EXT sequencing libraries}

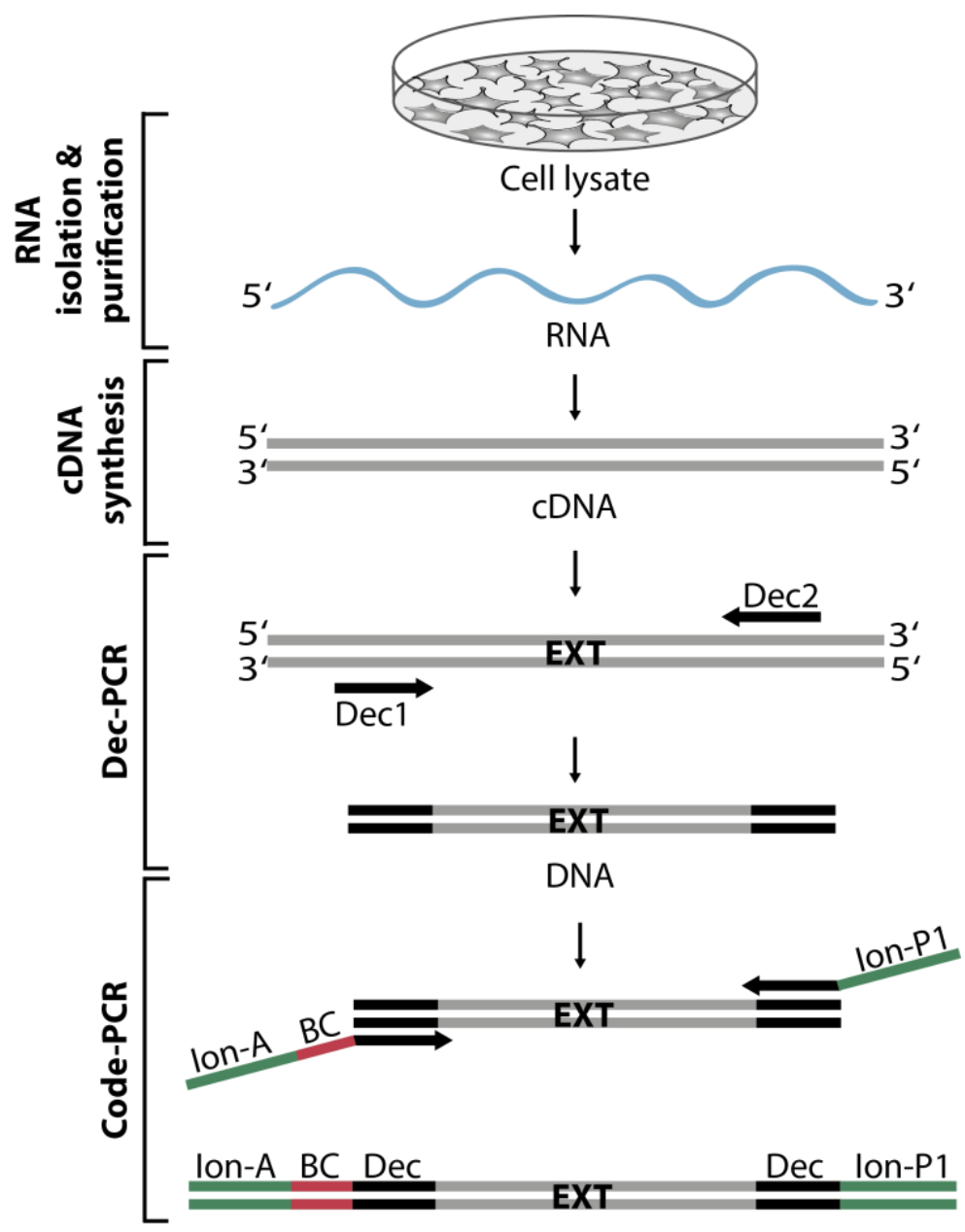

Final product

Figure 6: Workflow of EXT sequencing library preparation.

Total RNA is isolated and purified from cell lysates and converted into cDNA by reverse transcription. EXT sequences are amplified by a decoding PCR (Dec-PCR) using Dec1 and Dec2 primer. Ion-A adapters together with 13 mer barcodes $(B C)$ and lon-P1 adapters are added by Code-PCR.

\subsubsection{RNA isolation and purification}

EXT assays were performed in 6-well and 12-well plates using lipofectamine for DNA transfection. Dependent on the assay the cells were lysed 24-48 h after transfection with RLT buffer (500 $\mu \mathrm{l}$ for 6 -well, $200 \mu \mathrm{l}$ for 12 -well). To homogenize the lysate it was passed 5 times through a blunt 20-gauge needle $(0.9 \mathrm{~mm})$ fitted to a $1 \mathrm{ml}$ RNase free syringe. The RNA was purified with help of the RNeasy mini Kit from Qiagen according to the manufacture's protocol including an on column DNase digest (RNase free DNase kit from Qiagen). The RNA was eluted in $50 \mu$ and additional $40 \mu \mathrm{l}$ RNase free $\mathrm{H}_{2} \mathrm{O}$. The 
$90 \mu$ l RNA were additional in solution treated with $3 \mu$ RQ1 DNase (Promega) and $10 \mu \mathrm{l}$ of the appropriated $10 \mathrm{x}$ DNase reaction buffer for $30 \mathrm{~min}$ at $37^{\circ} \mathrm{C}$. After the DNase digest the RNA was purified again using the RNeasy mini kit and finally eluted in twice $50 \mu \mathrm{l}$ RNase free $\mathrm{H}_{2} \mathrm{O}$. The RNA concentration was measured using the picodrop.

\subsubsection{RNA precipitation}

RNA was precipitated with ammonium acetate salt and ethyl alcohol. To the $100 \mu \mathrm{l}$ purified RNA $50 \mu \mathrm{l} 7.5 \mathrm{M} \mathrm{NH}_{4} \mathrm{Ac}, 1 \mu \mathrm{l}$ glycogen $(20 \mathrm{mg} / \mathrm{ml})$ and $450 \mu \mathrm{l} 100 \%$ EtOH were added. The samples were mixed and centrifuged for $30 \mathrm{~min}$ at $13000 \mathrm{rpm}$. The RNA pellets were washed with $800 \mu \mathrm{l} 70 \% \mathrm{EtOH}$, air-dried and resuspended with RNase free $\mathrm{H}_{2} \mathrm{O}$. All samples were resuspended to the same concentration in at least $10 \mu \mathrm{l}$ $\mathrm{H}_{2} \mathrm{O}$ but maximal $1.5 \mu \mathrm{g} / \mu \mathrm{l}$.

\subsubsection{First strand cDNA synthesis}

The cDNA synthesis was performed with $1 \mu \mathrm{g}$ RNA using superscript III reverse transcriptase (Invitrogen). For all samples the equal amount of RNA was used. The cDNA synthesis was carried out in a total volume of $10 \mu \mathrm{l}$ by the following scheme:

$2 \mu \mathrm{RNA}$

$1 \mu$ l Random nanomer primer (120 pmol)

$1 \mu \mathrm{I}$ DTT

$2 \mu \mathrm{l} 5 \mathrm{x}$ First strand buffer

$0.5 \mu \mathrm{l}$ dNTP mix (10 mM each)

$2.5 \mu \mathrm{l} \mathrm{H} \mathrm{H}_{2} \mathrm{O}$

$1 \mu$ l Superscript III enzyme (200 U)

The samples were incubated for $10 \mathrm{~min}$ at $25^{\circ}$ for primer annealing followed by $30 \mathrm{~min}$ at $50^{\circ} \mathrm{C}$ for cDNA synthesis and $5 \mathrm{~min}$ at $85^{\circ} \mathrm{C}$ for heat inactivation of the enzyme. For all samples an additional negative control (-RT) was performed including all reaction agents except the superscript enzyme to control RNA purity with respect to plasmid DNA contaminations. 


\subsubsection{Composition of external calibrator mix}

For controlling and calibration of differences between samples within an experiment that can occur during cell handing, sample processing and sequencing a mix of additional EXT sequences were added as external calibrator EXTs (eCal-mix) in a constant amount to each sample at the step of EXT amplification (Dec-PCR). The eCal-mix consists of 15 5xUAS-TK-EXT plasmids with 3 plasmids each at a certain molecule number.

\begin{tabular}{lccc}
\multicolumn{3}{c}{ EXTs } & Molecules per $\boldsymbol{\mu l}$ \\
\hline 5xUAS-TK-E00055 & 5xUAS-TK-E00056 & 5xUAS-TK-E00057 & 10.000 \\
5xUAS-TK-E00058 & 5xUAS-TK-E00059 & 5xUAS-TK-E00060 & 3.162 \\
5xUAS-TK-E00061 & 5xUAS-TK-E00062 & 5xUAS-TK-E00063 & 1.000 \\
5xUAS-TK-E00064 & 5xUAS-TK-E00065 & 5xUAS-TK-E00066 & 316 \\
5xUAS-TK-E00067 & 5xUAS-TK-E00068 & 5xUAS-TK-E00069 & 100
\end{tabular}

\subsubsection{Amplification of EXT sequences}

As all EXT sequences are flanked by the same unique primer sequences the EXTs could be filtered out and amplified from the whole cDNA by PCR, called Decoding-PCR (Dec-PCR) (Figure 6). Additionally, $1 \mu \mathrm{l}$ of a 5xUAS-TK-EXT plasmid mix (eCal-mix) was added to each sample to introduce external calibrator EXT sequences. The Dec-PCR was performed for all samples including the $-\mathrm{RT}$ controls in a $20 \mu \mathrm{l}$ volume using the HotstarTaq Plus DNA polymerase (Qiagen).

Dec-PCR set up:

$1 \mu \mathrm{l}$ CDNA

$1 \mu \mathrm{l}$ eCal-mix

$2 \mu \mathrm{l}$ 10x CoralLoad buffer

$2 \mu \mathrm{l}$ dNTP mix ( $2.5 \mathrm{mM}$ each)

$0.1 \mu \mathrm{l}$ Forward Dec1 primer $(50 \mathrm{pmol} / \mu \mathrm{l})$

$0.1 \mu \mathrm{l}$ Reverse Dec2 primer (50 pmol $/ \mu \mathrm{l})$

$0.1 \mu \mathrm{l}$ HotStarTaq Plus polymerase

$13.7 \mu \mathrm{l} \quad \mathrm{H}_{2} \mathrm{O}$
PCR Program:

$95^{\circ} \mathrm{C} \quad \infty$

$95^{\circ} \mathrm{C} \quad 5 \mathrm{~min}$

$95^{\circ} \mathrm{C} \quad 30 \mathrm{sec}$

$\left.59^{\circ} \mathrm{C} \quad 30 \mathrm{sec}\right] 30$ cycles

$\left.72^{\circ} \mathrm{C} \quad 30 \mathrm{sec}\right]$

$10^{\circ} \mathrm{C} \infty$

To analyse the Dec-PCR $4 \mu \mathrm{l}$ of each sample was loaded on a $2 \%$ agarose gel. To indicate no Plasmid DNA contamination after the RNA purification the -RT samples should show no or only a slight band. 


\subsubsection{Attachment of Ion adapters and sample coding}

For sequencing with the Ion Torrent technology specific lon adapter sequences need to be added to the $3^{\prime}$ and $5^{\prime}$ end of the Dec-PCR fragments (Figure 6). On order to differentiate different samples in a sequencing run 13mer barcodes are introduced between $5^{\prime}$ Ion adapter and target sequence. The lon adapters and barcodes were added by PCR, called Code-PCR on the Dec-PCR product that was diluted 1:10 with $\mathrm{H}_{2} \mathrm{O}$.

Code-PCR set up:

$1 \mu \mathrm{l}$ Dec-PCR product (1:10 diluted)

$2 \mu \mathrm{l} 10 x$ CoralLoad buffer

$2 \mu \mathrm{l}$ dNTP mix (2.5 mM each)

$0.5 \mu$ l Forward Ion_A_code primer $(10 \mathrm{pmol} / \mu \mathrm{l})$

$0.5 \mu \mathrm{l}$ Reverse lon_P1 primer $(10 \mathrm{pmol} / \mu \mathrm{l})$

$0.1 \mu \mathrm{l}$ HotStarTaq Plus polymerase

$13.9 \mu \mathrm{l} \quad \mathrm{H}_{2} \mathrm{O}$
PCR Program:

$95^{\circ} \mathrm{C} \quad \infty$

$95^{\circ} \mathrm{C} \quad 5 \mathrm{~min}$

$95^{\circ} \mathrm{C} \quad 30 \mathrm{sec}$

$\left.58^{\circ} \mathrm{C} \quad 20 \mathrm{sec}\right] 10$ cycles

$72^{\circ} \mathrm{C} \quad 20 \mathrm{sec}$

$10^{\circ} \mathrm{C} \infty$

To analyse the Code-PCR $4 \mu$ l of each sample was loaded on a $2 \%$ agarose gel.

$10-12 \mu \mathrm{l}$ of each sample was pooled together and purified using the NucleoSpin Gel and PCR Clean-up kit (Macherey-Nagel). The final sample was eluted in $50 \mu$ l elution buffer and the concentration was measured using the picodrop.

\subsection{Next Generation Sequencing}

The prepared EXT template libraries were sequenced using the lon semiconductor sequencing technology by lon Torrent (Rothberg et al., 2011). The method is based on the detection of released hydrogen ions during DNA replication. The incorporation of a deoxyribonucleotide triphosphate (dNTP) in a new synthesised strand leads to the formation of a covalent bond and the release of pyrophosphate and a hydrogen ion. This happens only if the dNTP is complementary to the leading strand. The released positively charged hydrogen ion causes a $\mathrm{pH}$ change of the solution resulting in a voltage change that is detected by lon sensors. The advantage of this method is the use of unmodified nucleotides and the lack of any optics. 
The sequencing device consists of two parts, the sequencing instrument itself and an Ion semiconductor chip. An Ion chip contains a high-density array of micro-sized wells where the sequencing reaction takes place. Each of these wells is loaded with a different single-stranded DNA template and a DNA polymerase. During sequencing reaction the chip is flooded sequentially with one nucleotide after another. When a complementary dNTP is incorporated sensors on the lon chip detect the $\mathrm{pH}$ change caused by the released hydrogen ion. The sequencing instrument comprises a fluidic system to control the sequencing reagents and nucleotide flows over the chip and record and process the detected signals. Actually there are two sequencing instruments, the Ion Personal Genome Machine (PGM) and the Ion Proton and four different Ion chips available, which differ in their sequencing capability. Ion 314, 316 and 318 chips run on the Ion PGM and the Ion PI chip on the Ion Proton. Which chip type should be used for an experiment is dependent on the complexity of different sequences and the desired coverage of reads per individual sequence. The different chip types and their specifications are listed in Table 2. All GPCR activation assays were sequenced on the lon PGM using 318 chips and the GPCR signalling assay on the lon Proton using the PI chip.

Table 2: Overview of Ion Torrent sequencing devices and chips.

\begin{tabular}{l|ccc|c} 
Sequencer & \multicolumn{3}{|c|}{ Ion PGM } & Ion Proton \\
\hline Chip & 314 & 316 & 318 & PI \\
\hline Total output & up to $100 \mathrm{Mb}$ & up to $1 \mathrm{~Gb}$ & up to $2 \mathrm{~Gb}$ & up to $10 \mathrm{~Gb}$ \\
\hline Reads & $400.000-700.000$ & $2-3$ Mio & 5-7 Mio & 70-100 Mio \\
\hline Read length & \multicolumn{3}{|c|}{ 200-400 Bases } & 200 Bases
\end{tabular}




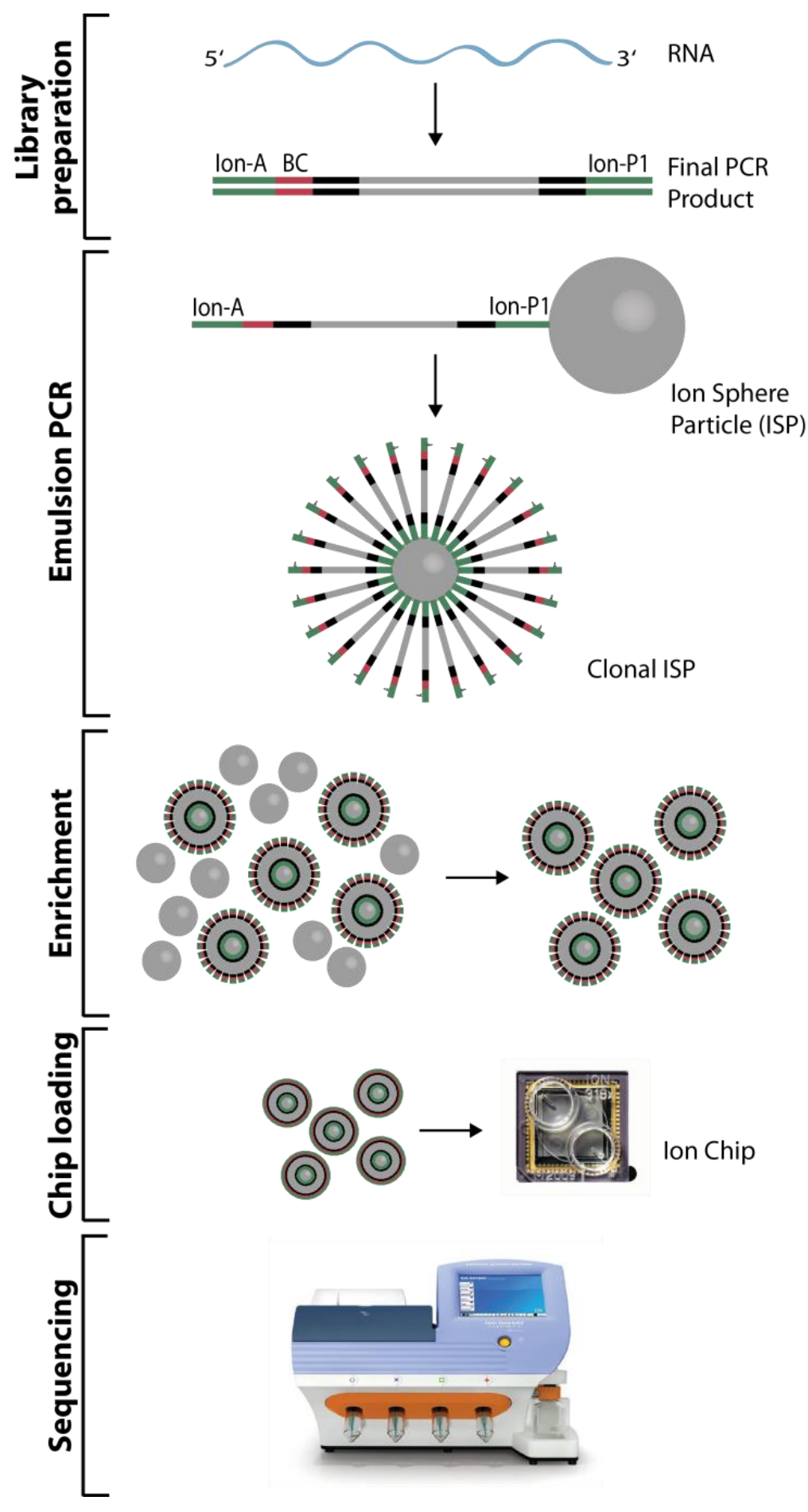

Figure 7: Workflow of Next Generation Sequencing by lon Torrent technology.

During library preparation isolated RNA is converted into DNA and flanked by lon adapters (Ion-A and Ion-P1) and a unique barcode per sample. Next, the fragments are bound to lon Sphere Particles (ISPS) and amplified by an emulsion PCR. Template-positive ISPs are then isolated and enriched. In the next step the prepared template is loaded on an Ion Chip and sequenced using the lon PGM or Proton. 


\subsubsection{Preparation of sequencing templates}

After library preparation the fragments are attached to Ion Sphere Particles (ISPs) and amplified by emulsion PCR (emPCR) using the Ion One Touch system. The fragments to be sequenced are mixed with PCR reagents and ISPs covered with primers complementary to the Ion-P1 adapter sequence on the fragments. This aqueous solution is then mixed with oil to form an emulsion of microdroplets. For clonal amplification the concentration of ISPs and fragments are chosen in a way that each microdroplet contain only one ISP and one fragment.

For template preparation of EXT libraries $23 \mathrm{pM}$ of the final sample was used. For this, the final purified sample was diluted to a concentration of $2-5 \mathrm{ng} / \mu \mathrm{l}$ and the concentration measured with the Qubit 2.0 Fluorometer (Invitrogen). The sample was then diluted to $23 \mathrm{pM}$ and $25 \mu \mathrm{l}$ were used for emPCR. After clonal amplification template-positive ISPs were enriched using the Ion One Touch ES device. Because of the use of biotinylated primers during emPCR streptavidin-coupled magnetic beads bind only template-positive ISPs.

All template preparations and enrichments were done according to the manufactures protocols for the lon PGM ${ }^{\mathrm{TM}}$ Template OT2 200 Kit, Ion PGM ${ }^{\mathrm{TM}}$ Template OT2 400 Kit or the Ion $\mathrm{PI}^{\mathrm{TM}}$ Template OT2 200 v3 Kit respectively.

\subsubsection{Sample sequencing on Ion Torrent sequencer}

Before starting a sequencing run, the lon sequencer needs to be initialized. Both devices, the Ion PGM and the Ion Proton are initialized by the same procedure. This means the preparation of wash-solutions and nucleotides. After manual preparation of the two wash buffers $W 1$ and W2 the instrument is mixing these buffers until the W2 buffer reaches a $\mathrm{pH}$ between 7.45-7.7 according to the reference buffer W3. The final W2 buffer then adjust the four nucleotides.

After initialization sequencing primers are annealed to the template-positive ISPS carrying the EXT fragments. The final sample is then loaded together with a polymerase onto an Ion chip and the sequencing run started. During the run the chip is flooded sequentially with one nucleotide after another. Between the flows the chip is washed with W2 buffer to remove unattached dNTPs. 
The sequencing runs were done according to the manufactures protocols for the lon $\mathrm{PGM}^{\mathrm{TM}}$ Sequencing 200 v2 Kit, lon $\mathrm{PGM}^{\mathrm{TM}} \mathrm{Hi}^{-\mathrm{Q}^{\mathrm{TM}}}$ Sequencing Kit or lon $\mathrm{PI}^{\mathrm{TM}}$ Sequencing 200 v3 Kit.

\subsubsection{Analysis of sequencing data}

Raw data analyses were performed by Dr. Sven Wichert (Molecular Neurobiology, Department of Psychiatry, LMU Munich) using BLAST algorithm and in-house developed R scripts. Raw sequencing reads were normalized to total read numbers of samples and additionally to average reads of internal and external calibrator EXTs. Final normalized data is given either as raw fold changes (relative differences) or log2 fold changes between samples.

In the GPCR signalling assays for each sensor a variance factor was calculated by variance analyses based on the log2 fold change values. For filtering all sensors with a variance factor under 0.03 were excluded from further analysis and heatmap plotting. The hierarchical clustering was done by the average method using $\mathrm{R}$.

\subsection{Cloning of GPCRs}

All GPCRs except of ADRB3 and DRD4 were first PCR amplified from human brain cDNA library using primers complementary to the $5^{\prime}$ and $3^{\prime}$ end of the specific GPCR coding sequence and carrying sequences for attB1 and attB2 recombination sites. The PCR products were then recombined within a Gateway BP reaction to create pENTR shuttle clones. DRD4 was purchased from Bio Basic Inc. as synthesised linear sequence with already attached attB1 and attB2 sites. ADRB3 was purchased from Source Bioscience as pENTR shuttle vector. All GPCR entry vectors were sequence verified. After verification the entry vectors were recombined within a LR reaction into the destination vector PTag4C_ST_X-V2R-NTEV-tevS-GV to obtain the final expression vectors of GPCR-V2R-NTEV-tevS-GV fusion constructs. The final constructs were verified by diagnostic restriction enzyme digest. 


\subsubsection{Modification of GPCRs}

Due to a low expression rate and functionality in split TEV assays of some GPCRs the signal peptide for cell membrane localisation was either exchanged or an additional signal peptide was added for these receptors. A list of modified GPCRs is depicted in Table 3.

For the modification by changing the signal peptide the native signal peptide sequence was determined using the program http://www.predisi.de/. The signal peptide was changed by the signal sequence of the mouse Ig K-chain V-J2-C proteins (Figure 8A). The database on http://bp.nuap.nagoya-u.ac.jp/sosui/sosui_submit.html was used to ensure a space of at least three amino acids in front of the first transmembrane domain. The in this way modified GPCRs were called as "HOOK-GPCRs". The validation was done in my diploma thesis 2010.

Another modification method to promote membrane localization was the adding of a HA-Flag Tag at the N-terminus of the GPCR (Figure 8B) (Guan et al., 1992; Kroeze et al., 2015). These GPCRs were called as HA-Flag-GPCR or HF-GPCR.

Table 3: Overview of modified GPCRs.

\begin{tabular}{ll} 
Native GPCR & Modified GPCR \\
\hline HTR1A & HA-Flag-HTR1A \\
\hline HTR2A & HOOK-HTR2A \\
\hline HTR7 & HA-Flag-HTR7 \\
\hline DRD3 & HA-Flag-DRD3 \\
\hline DRD4 & HA-Flag-DRD4 \\
\hline SSTR1 & HOOK-SSTR1
\end{tabular}

A Mouse Ig k-chain V-J2-C signal peptide

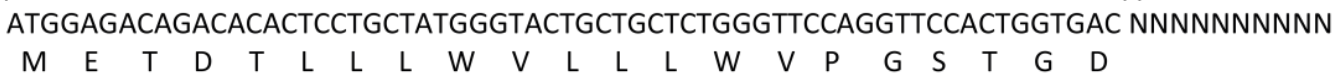
B HA-Flag Tag GPCR ATGAAGACGATCATCGCCCTGAGCTACATCTTCTGCCTGGTATTCGCCGACTACAAGGACGATGATGACAAG NNNNNNNNNN $\begin{array}{lllllllllllllllllllllllll}M & K & T & \text { I } & \text { I } & A & L & S & Y & \text { I } & F & C & L & V & F & A & D & Y & K & D & D & D & D & K\end{array}$

Figure 8: Modification of GPCR signal peptides.

The native signal peptide sequence was modified $(A)$ by exchange of the $5^{\prime}$ signal sequence by the signal sequence of the mouse Ig K-chain V-J2-C protein or (B) by adding a HA-Flag Tag sequence at the $5^{\prime}$ end. 


\subsection{Cloning of EXT reporters}

\subsubsection{0xUAS-EXT reporters for GPCR activation assays}

The 10xUAS-EXT reporters were generated by multisite Gateway recombination. Ordered 5xUAS oligonucleotides were clustered by PCR and recombined in a BP reaction to obtain a 10XUAS_pENTR (L1/L4) shuttle vector. This shuttle vector was recombined with pENTR (L4/L3) clone encoding the adenovirus major late minimal promoter (MLP) and multiple unique pENTR-EXT (L3/L2) vectors. In total 67 10xUAS-EXT reporters were cloned and sequence verified.

\subsubsection{Sensor-EXT reporters for GPCR signalling assay}

All sensor-EXT reporters (cis-EXT and promoter-EXT reporters) were cloned within a one reaction multisite Gateway recombination of three fragments into a final pAAV vector backbone. Clustered cis-elements were either synthesized by GenScript as pENTR (L1/L4) shuttle vectors or amplified by PCR from available reporter constructs and recombined within a Gateway BP reaction to create attL1-attL4-pENTR (L1/L4) shuttle clones. Each cis-element was clustered 6-18x depending on the sequence length. Endogenous promoter fragments were PCR amplified from genomic DNA and recombined within a BP reaction to generate attL4-attL3-pENTR shuttle vectors. The primers for PCR amplifications were placed in a way that a 1-1.5 kb long region upstream of the transcription start site was generated. Each of the shuttle clones were than recombined either with pENTR (L4/L3) clones encoding either the adenovirus major late minimal promoter (MLP; cis-elements) or with pENTR (L1/L4) carrying a non-coding "dummy sequence" (first $200 \mathrm{bp}$ of the enhanced green fluorescence protein, sEGFP) together with multiple unique pENTR-EXT (L3/L2) vectors. For some cis-elements different versions of transcription factor binding sequences were existent so that in total 69 cis-elements and 24 promoters were cloned. The cis- and promoterEXT constructs were cloned for the most part by Dr. Ben Brankatschk (Molecular Neurobiology, Department of Psychiatry, LMU Munich). All obtained final reporter constructs were sequence verified. The different cis-elements and promoters are listed in Table 4 and a complete list of all reporter plasmids is shown in Table 1. 
Table 4: List of cis-elements and endogenous promoters for sensor-EXT reporters.

\begin{tabular}{|c|c|c|}
\hline Cis-element & Transcription factor & Pathway \\
\hline AARE & ATF2-4 & Amino acid deprivation \\
\hline AR-RE & $A R$ & Androgens \\
\hline ARE & $\operatorname{NRF}(2)$ / NFE2L2 & Antioxidant response \\
\hline Npas4-RE & Npas4 & $\mathrm{Ca}^{2+}$ - signalling \\
\hline SARE & CREB, MEF2, SRF & $\mathrm{Ca}^{2+}-$, cAMP- signalling \\
\hline NFAT-RE & NFAT & $\mathrm{Ca}^{2+}-, \mathrm{cAMP}-$ signalling \\
\hline CRE & CREB & cAMP - signalling \\
\hline E2F-RE & $\mathrm{E} 2 \mathrm{~F}$ & Cell cycle \\
\hline RB-RE & $\mathrm{RB}$ & Cell cycle \\
\hline SREBP-RE & SREBP & Cholesterol \\
\hline ER-RE & ER / ESR1 & Estrogen \\
\hline HSE & $\mathrm{HSF}$ & Heat shock \\
\hline GLI-RE & GLI & Hedgehog \\
\hline TEAD4-RE & TEAD & Hippo \\
\hline HRE & HIF1a & Hypoxia \\
\hline ISRE & IRF1, STAT1-2-IRF9 & JAK - STAT \\
\hline STAT-RE & STAT1/3/4 & JAK/STAT proliferation \\
\hline NFkB-RE & NFkB & MAPK \\
\hline AP1 & AP1 & MAPK \\
\hline EGR1-RE & EGR1 & MAPK \\
\hline ELK1-RE & ELK1 & MAPK \\
\hline SRE & SRF, ETS / ELKS & MAPK \\
\hline SRF-RE & SRF & MAPK \\
\hline E-box & Myc & Metabolic and circadian control \\
\hline CEBP-RE & CEBP-a & Metabolism \\
\hline SP1-RE & SP1 & Metabolism \\
\hline MRE & MTF1 & Metal response \\
\hline Notch-RE & RBP-JK / RBPJ / CBF1 & Notch \\
\hline GR-RE & GR / NR3C1 & Nuclear steroid receptors \\
\hline KLF4-RE & KLF4 & Stem cells \\
\hline NANOG-RE & NANOG & Stem cells \\
\hline OCT4-minP & OCT4 & Stem cells \\
\hline OCT4-RE & OCT4 & Stem cells \\
\hline SOX2-OCT4-cRE & SOX2-OCT4 & Stem cells \\
\hline SOX2-RE & SOX2 & Stem cells \\
\hline p53-RE & p53 & Apoptosis \\
\hline FOXO-RE & FOX01/3 & PI3K-AKT \\
\hline IRS & FOXO & PI3K-AKT \\
\hline GATA-RE & GATA & Proliferation \\
\hline Myc_E-box & MYC, MAX, MAD & Proliferation \\
\hline RARE & RAR::RXR all & Retinoic acid \\
\hline SMAD-RE & SMAD1-5 & TGF $\beta$ \\
\hline ERSE & NF-Y, ATF6, XBP1, YY1 & Unfolded protein response \\
\hline UPRE & ATF6, XBP1 & Unfolded protein response \\
\hline YY1-RE & YY1 & Unfolded protein response \\
\hline Wnt-RE & TCF-LEF & Wnt/ $\beta$-catenin \\
\hline NRSE & NRSF & $\begin{array}{l}\text { Neural stem cells, suppression of } \\
\text { neurogenesis }\end{array}$ \\
\hline
\end{tabular}


Endogenous Promoter

TP53INP1

TP73

Npas4

PPP1R15A

TNFA

CXCL1

IL6

IL8

HSPA1A

DUSP1

DUSP5

EGR1

EGR2

FOS

FOSB

JUN

NR4A1

NFKB2

hDLG4_1.ATG

sDLG4

EIF2AK2

PSMB9

\section{Pathway}

Apoptosis

Apoptosis

$\mathrm{Ca}^{2+}$ - signalling

cAMP-signalling

cAMP-signalling

Cytokine signalling, immune response Cytokine signalling, immune response Cytokine signalling, immune response Heat shock

MAPK

MAPK

MAPK

MAPK

MAPK

MAPK

MAPK

MAPK

NFkB / cytokines

Nrg1 back signalling

Nrg1 back signalling

STAT1, interferon

STAT1, interferon 


\section{Results}

\subsection{GPCR activation assays}

\subsubsection{Principle of GPCR split TEV assay}

The stimulation-dependent interaction of GPCR and $\beta$-arrestin was used to set up split TEV assays for monitoring a ligand-dependent GPCR activation.

The split TEV technique is based on the complementation of two inactive fragments of the tobacco etch virus (TEV) protease (NTEV and CTEV) to reconstitute the functional protease as a result of a protein-protein interaction (Wehr et al., 2006), in this case a GPCR and $\beta$-arrestin. To do this, amino acids $343-371$ of the C-terminal intracellular domain of the human arginine vasopressin receptor 2 (AVPR2) (abbreviated V2R) were fused to the C-terminal end of a candidate GPCR, followed by the NTEV fragment (amino acid 1-118), the TEV cleavage site ENLYFQ'G (tevS) and the artificial transcription factor Gal4-VP16 (GV) (Figure 9A). The V2R- tail was added to improve the stability between GPCR and arrestin upon receptor activation, because of the highly variable affinity of the different GPCRs to arrestin. The specificity of the ligands to the GPCRs and the downstream signalling is not affected (Barnea et al., 2008; Oakley et al., 2000). The C-terminal TEV fragment (amino acids 119-221, S219P mutant) was fused to a truncated $\beta$-arrestin 2 lacking the entire $C$-terminal tail (amino acids 383-410) (Figure 9A). This truncation exhibits stronger stimulation-dependent receptor desensitisation and therefore enhances the assay sensitivity compared with wild type $\beta$-arrestin (Djannatian et al., 2011; Kovoor et al., 1999). The CTEV fragment was further optimised and carries the stabilising point mutation S219P (Kapust et al., 2001) and is truncated after amino acid 221 to remove the auto-inhibitory C-terminal tail (Nunn et al., 2005).

After ligand-dependent GPCR activation, $\beta$-arrestin is recruited to the receptor and both TEV fragments come into close proximity. The reconstituted TEV protease cleaves at the tevS, causing GV to be released, which translocates into the nucleus. There, GV activates a transcriptional reporter by binding to clustered Gal4-responsive upstream activating sequence (UAS) elements (Figure 9B). 
Either firefly luciferase or EXT molecular barcode reporters were used as read out. The

firefly luciferase readout was used both in initial experiments to set up the split TEV technique for a selection of GPCRs and in later validation experiments.

A \begin{tabular}{llll|l|lll} 
CMV attB1 & GPCR & attB2 V2R & NTEV & tevs & GV & pA & GPCR-V2R-NTEV-tevs-GV \\
\hline
\end{tabular}

B

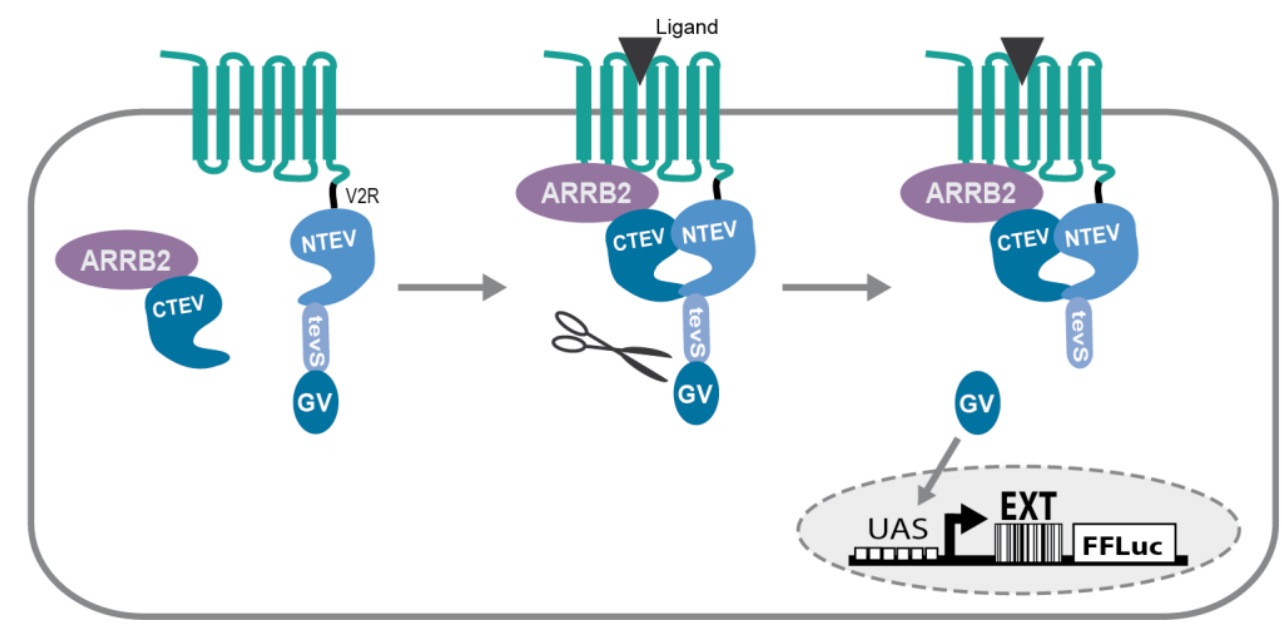

Figure 9: GPCR split TEV constructs and principle of GPCR split TEV assays.

(A) Structure of split TEV constructs for GPCR activation assays. GPCR-V2R-NTEV-tevS-GV constructs are composed of the GPCR fused to the C-terminal domain of the arginine vasopressin receptor 2 (V2R) followed by the N-terminal TEV protease fragment (NTEV, aa 1-118), the TEV cleavage site (tevS) and the Gal4-VP16 transcription factor (GV). $\beta$-arrestin2 $\triangle$-CTEV constructs harbours the truncated human $\beta$-arrestin 2 (ARRB2 $\triangle 383$, aa 1-383) fused to the C-terminal TEV protease fragment (CTEV, aa 119-221). GPCR-V2R-NTEV-tevS-GV and $\beta$-arrestin2 $\triangle$-CTEV fusion proteins are expressed under the control of a cytomegalovirus promoter (CMV). attB1 and attB2 designate recombination sites. (B) Schematic representation of GPCR split TEV assays. The ligand-dependent receptor activation results in the recruitment of $\beta$-arrestin to the GPCR and the reconstitution of the protease activity. TevS is cleaved (indicated by the scissors) and the released GV translocates to the nucleus to activate reporter gene (i.e. firefly luciferase, FFLuc) or EXT barcode reporters by binding to upstream activating sequence (UAS) elements. 


\subsubsection{Cloning of GPCR split-TEV fusions}

21 selected GPCRs were fused to the V2R-NTEV-tevS-GV moiety. For some GPCRs, HOOK or HA-FLAG tags were fused to the N-terminus of the GPCRs to increase expression levels in living cells. Full descriptions of the cloning procedure can be found at chapter 4.5. Details for all GPCRs used are depicted in Table 5 including the Reference Sequence (RefSeq) numbers provided by the National Center for Biotechnology Information (NCBI).

Table 5: Overview of all GPCR split TEV fusion constructs used in GPCR activation assays.

Table listed all GPCRs used in the following assays as denoted in the main text, the full fusion protein constructs and the corresponding Reference Sequence (RefSeq) numbers.

\begin{tabular}{|c|c|c|c|}
\hline $\begin{array}{l}\text { Receptor } \\
\text { subgroup }\end{array}$ & $\begin{array}{l}\text { GPCR } \\
\text { (denoted in text) }\end{array}$ & Full fusion protein & RefSeq \\
\hline \multirow{5}{*}{$\begin{array}{l}\text { Serotonin } \\
\text { receptors }\end{array}$} & HTR1A & HA-FLAG-HR1A-V2R-NTEV-tevS-GV & NM_000524 \\
\hline & HTR2A & HOOK-HTR2A-V2R-NTEV-tevS-GV & NM_000621 \\
\hline & HTR4 & HTR4-V2R-NTEV-tevS-GV & NM_001040169 \\
\hline & HTR5A & HTR5A-V2R-NTEV-tevS-GV & NM_024012 \\
\hline & HTR7 & HA-FLAG-HTR7-V2R-NTEV-tevS-GV & NM_000872 \\
\hline \multirow{6}{*}{$\begin{array}{l}\text { Dopamine } \\
\text { receptors }\end{array}$} & DRD1 & DRD1-V2R-NTEV-tevS-GV & NM_000794 \\
\hline & & & NM_016574 \\
\hline & DRD2 & DRD2-V2R-NTEV-tevS-GV & (short isoform) \\
\hline & DRD3 & HA-FLAG-DRD3-V2R-NTEV-tevS-GV & BC128123 \\
\hline & DRD4 & HA-FLAG-DRD4-V2R-NTEV-tevS-GV & NM_000797 \\
\hline & DRD5 & DRD5-V2R-NTEV-tevS-GV & NM_000798 \\
\hline \multirow{3}{*}{$\begin{array}{l}\alpha \text {-adrenergic } \\
\text { receptors }\end{array}$} & ADRA1A & ADRA1A-V2R-NTEV-tevS-GV & NM_033303 \\
\hline & ADRA2B & ADRA2B-V2R-NTEV-tevS-GV & NM_000682 \\
\hline & ADRA2C & ADRA2C-V2R-NTEV-tevS-GV & NM_000683 \\
\hline \multirow{2}{*}{$\begin{array}{l}\beta \text {-adrenergic } \\
\text { receptors }\end{array}$} & ADRB2 & ADRB2-V2R-NTEV-tevS-GV & NM_000024 \\
\hline & ADRB3 & ADRB3-V2R-NTEV-tevS-GV & NM_000025 \\
\hline \multicolumn{4}{|l|}{ Histamine } \\
\hline receptor & $\mathrm{HRH} 1$ & HRH1-V2R-NTEV-tevS-GV & NM_000861 \\
\hline \multirow{2}{*}{$\begin{array}{l}\text { Vasopressin } \\
\text { receptors }\end{array}$} & AVPR1A & AVPR1A-V2R-NTEV-tevS-GV & NM_000706 \\
\hline & AVPR2 & AVPR2-V2R-NTEV-tevS-GV & NM_000054 \\
\hline \multirow{3}{*}{$\begin{array}{l}\text { Somatostatin } \\
\text { receptors }\end{array}$} & SSTR1 & SSTR1-V2R-NTEV-tevS-GV & NM_001049 \\
\hline & SSTR2 & SSTR2-V2R-NTEV-tevS-GV & NM_001050 \\
\hline & SSTR3 & SSTR3-V2R-NTEV-tevS-GV & NM_001051 \\
\hline
\end{tabular}




\subsubsection{EXT barcode reporters}

For monitoring the receptor activation of multiple GPCRs simultaneously in one experiment, a new class of reporter constructs was generated (Figure 10). Expressed oligonucleotide tags (EXTs) are unique barcode sequences that were used to replace classical reporter proteins as read out. To generate a large number of unique EXT barcode reporters, a multi-fragment recombination cloning strategy was applied (Multisite Gateway Recombination Technology). A library of shuttle vectors carrying different EXTs were recombined together with two shuttle vectors harbouring a transcription factor binding site and a minimal promoter into a firefly luciferase reporter backbone vector. The final reporter constructs consist of a 10 times clustered upstream activating sequence (10xUAS) element as binding sequence for the artificial transcription factor GV followed by a cytomegalovirus minimal promoter (CMVmin), a unique EXT and the firefly luciferase sequence (Figure 10A). Non-variable regions flank the variable EXT sequence as primer binding sites (Dec1 and Dec2) needed during the analysis of expressed EXTs. Because of the dual expression of EXT and luciferase after reporter activation the reporters could be used in multiplexed EXT assays (denoted as 10xUAS-EXT reporters) or in single luciferase assays (denoted as 10xUAS-FFLuc reporters). For multiplexed EXT assays, a construct library of about 70 unique 10xUAS-EXT barcode reporters was prepared (Figure 10B).

A

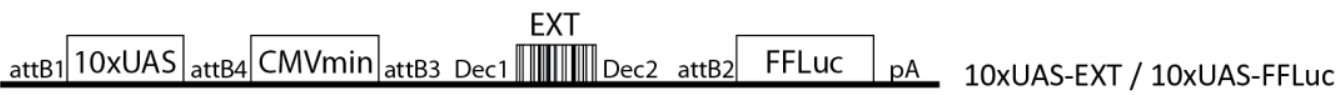

B

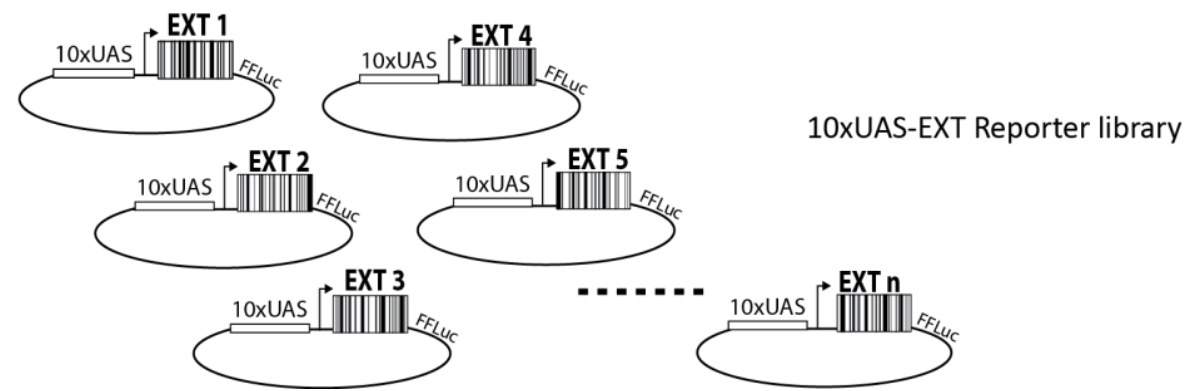

Figure 10: Structure of EXT barcode reporter constructs and library.

(A) Modular reporter construct. By a one-reaction recombination the following three fragments were introduced in front of the firefly luciferase gene (FFLuc): the GV transcription factor binding site (10xUAS), a cytomegalovirus minimal promoter (CMVmin) and an EXT flanked by "decoding" primer binding sites (Dec1 and Dec2). attB1, attB2, attB3 and attB4 are the recombination site of the three fragments. Naming of the reporter varies between applications, 10xUAS-EXT in multiplexed EXT assays and 10xUAS-FFLuc in single luciferase assays. (B) Library of multiple unique EXT barcode reporters. 
The general performance of the reporters in response of the Gal4-VP16 transcription factor (GV) was determined in a luciferase assay by cotransfection with a constitutively expressed GV (Figure 11). The reporter showed significant responses of 70-fold with 1ng GV and 90 -fold with $5 \mathrm{ng}$ GV in PC12 cells after $24 \mathrm{~h}$. In contrast the signals remained at the reporter background level when cells were treated with mainly used agonists of GPCR activation assays in PC12 cells (Figure 11) as well as in U2OS cells, which indicate no direct interference of receptor stimulating substances with the reporter system.

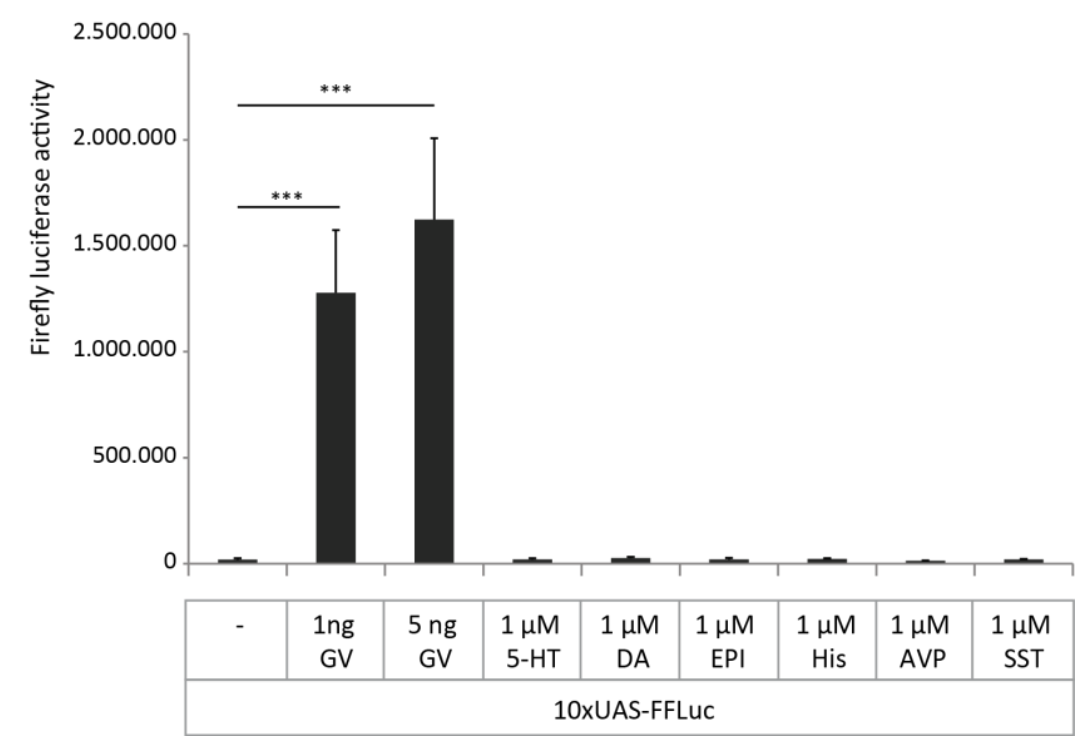

Figure 11: Control of reporter inducibility and potential substance interference.

Luciferase based functional test of 10xUAS reporter performance. PC12 tetOFF cells were transfected with $10 \mathrm{ng}$ of 10xUAS-FFLuc reporter plasmid alone or together with $1 \mathrm{ng}$ and $5 \mathrm{ng}$ GV, respectively. Both GV cotransfections show significant high activation signals. To monitor potential substance interference on the reporter performance cells transfected with reporter alone were treated for $6 \mathrm{~h}$ with the GPCR agonists serotonin (5-HT), dopamine (DA), epinephrine (Epi), vasopressin (AVP) and somatostatin (SST). The reporter shows no changes in signal intensity compared to the background signal of untreated reporter. Luciferase data are given as means \pm standard deviations $(n=6) .{ }^{*} p \leq 0.05$; $* * \mathrm{p} \leq 0.01 ;{ }^{*} \mathrm{p} \leq 0.001$ (two-sided t-test). 


\subsubsection{Workflow of GPCR split TEV assays}

The general workflow and timeline for agonist and antagonist treated assays is depicted in Figure 12. All assays for monitoring GPCR activity were done by transient transfection of the assay components. The method of transfection, on-plate or in-solution, was dependent on the assay type. In luciferase-based assays, cells were on-plate transfected whereas cells in most EXT-based assays were transfected in-solution. For both methods, cells were incubated with the transfection mix for $2 \mathrm{~h}$. For expression of the assay components and the receptor localisation at the membrane, cells were cultivated after transfection in their respective maintenance medium for 20h. After this expression phase maintenance medium was changed by assay medium with no or reduced fetal bovine serum (FBS), dependent on the cell line. The serum-starvation leads to a reduction of basal cell activity and thereby enhances a potential stimulation response after applying a stimulus. Cells were usually serumstarved over-night.

The following stimulation phase varied between the applied substances and a point analysis (agonist and drug assays) or time analysis (kinetic assays). In agonist assays, an agonist diluted in fresh assay medium was added to the cells and incubated for $6 \mathrm{~h}$. Cells were then lysed and analysed either by luciferase readings or EXT sequencing. To monitor inhibition effects of drugs/antagonists, the drug/antagonist was added to the cells first and pre-incubated for $1 \mathrm{~h}$ before adding the corresponding agonist for further $6 \mathrm{~h}$ incubation.

In kinetic assays the stimulation response of a receptor was measured by live monitoring the luciferase activity over time. After adding an agonist the cells were incubated for $24 \mathrm{~h}$ or longer in a lumicycler (LumiCycle 32 from ActiMetrics) permanently recording luciferase activity. 


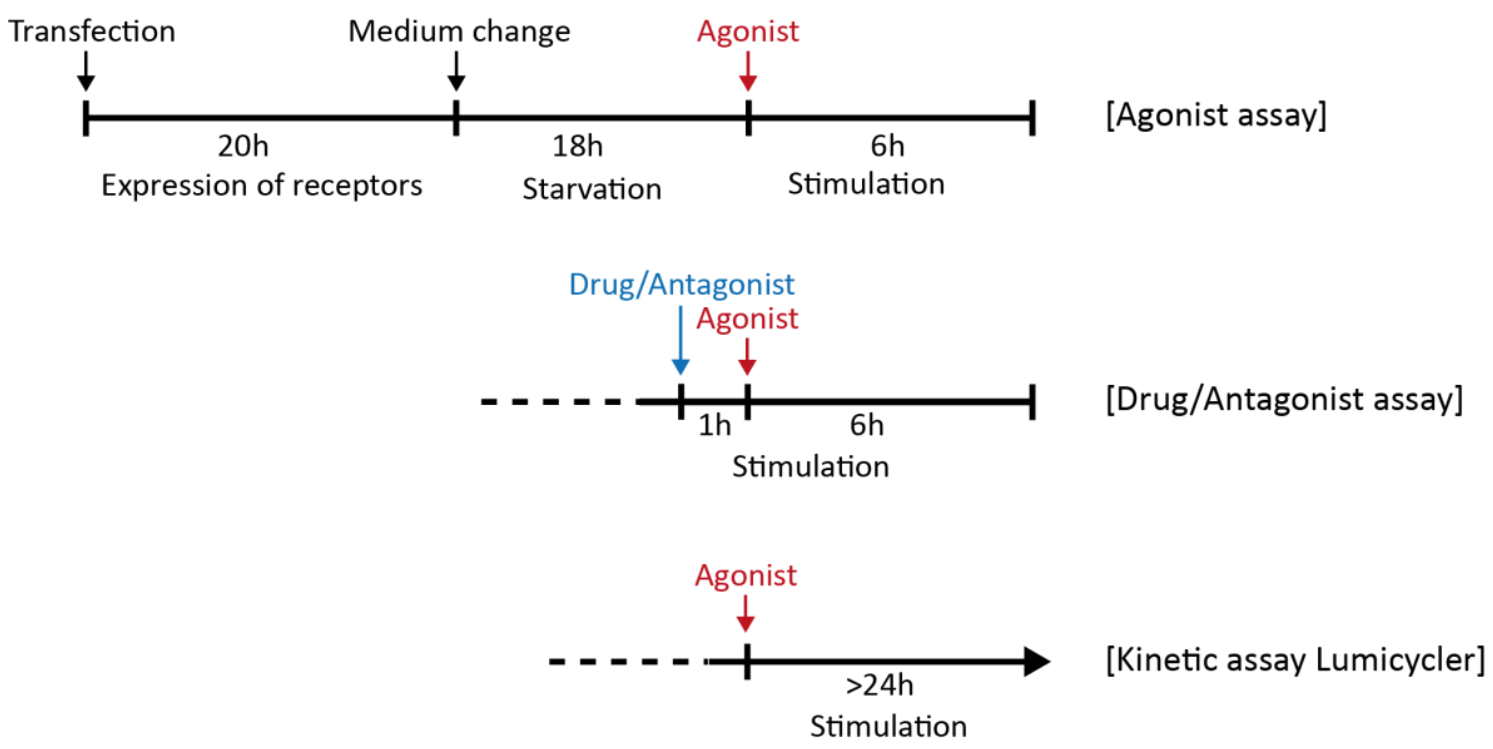

Figure 12: Timeline and workflow of different types of GPCR split TEV assays.

In all assay types cells were transiently transfected followed by an expression phase of 20h. Afterwards, the medium was changed by serum reduced assay medium for reducing basal cell activity (starvation period). In agonist assays, an agonist was added and incubated for $6 \mathrm{~h}$ before lysis. In drug assays, cells were pre-incubated with a drug/antagonist for $1 \mathrm{~h}$ before adding the corresponding agonist for additional $6 \mathrm{~h}$ followed by lysis. In kinetic assays, after addition of an agonist cells were incubated in a lumicycler where the luciferase activity was monitored over time.

\subsubsection{GPCR activation kinetics}

EXT-based and standard luciferase assays are time point analyses at the point of lysis. To determine the optimal point of lysis and thus the length of stimulation phase the kinetic of receptor activation in split TEV assays was monitored. This was done by incubating transfected cells in an online luciferase assay, recording the luciferase activity from the beginning of starvation (Figure 13). Six different GPCRs (2 serotonin, 2 dopamine and 2 adrenergic receptors) were measured in duplicates. During the starvation phase the luciferase readings of all GPCRs decrease, displaying the reduction of basal cell activity. After applying GPCR corresponding agonists at time point 0 the stimulated samples show an increase in luciferase activity. Over time the readings are decreasing to level of unstimulated samples. The peaks of activity are between $2.5 \mathrm{~h}$ stimulation for DRD2 and 9h for ADRA1A (Figure 13, lower square). The average induction rates (the maximal signal of stimulated samples divided by the 
corresponding unstimulated background) are between 1.5-fold for DRD2 and 17-fold for HTR2A.

Based on the kinetics, the period of stimulation for time point assays was set to $6 \mathrm{~h}$ as average for all selected GPCRs.

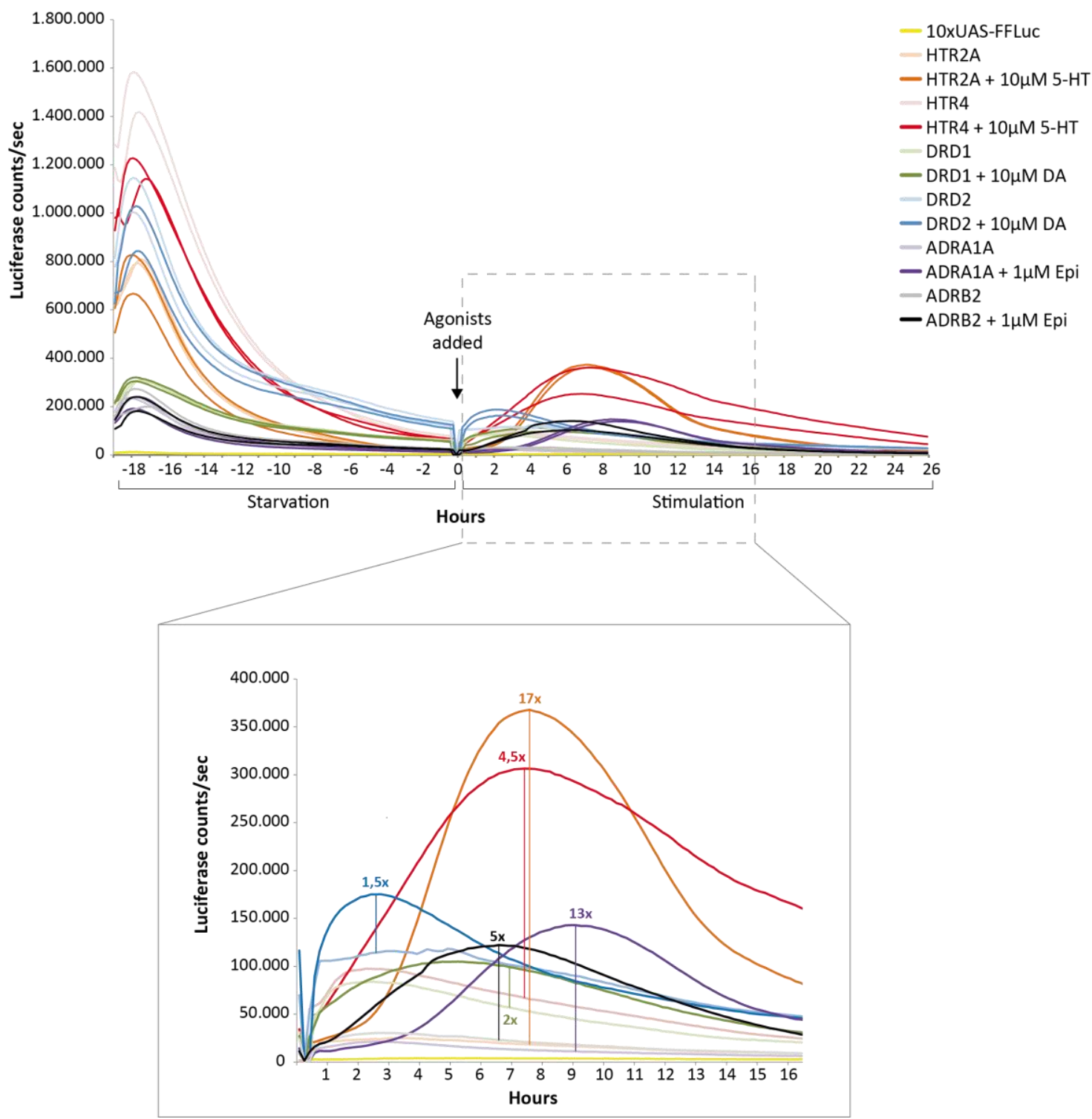

Figure 13: Kinetic measurement of ligand dependent GPCR activation.

Receptor activation kinetics were measured for the GPCRs HTR2A, HTR4, DRD1, DRD2, ADRA1A and ADRB2. PC12 tetOFF cells were transfected with GPCRs (fused to V2R-NTEV-tevS-GV) and ß-arrestin2 $\Delta$ CTEV and the luciferase activity measured constantly during starvation phase (18h) and after application of agonists at indicated concentrations (stimulation phase, 26h). Decreasing signals during starvation phase reflect the reduction of cell activity. Luciferase readings of stimulation phase are highlighted in the box. For each GPCR the means of two replicates of unstimulated and stimulated samples are displayed. Agonists: $5 \mathrm{HT}$, serotonin; DA, dopamine; Epi, epinephrine. 


\subsubsection{Luciferase-based evaluation of GPCR activation}

First, single luciferase assays were performed to monitor GPCR activities. Successively, this data was analysed for efficient combination of the EXTassay technique and the split TEV method, thereby allowing experimental setups for multiplexed cell-based assays.

21 GPCRs were selected from the serotonin, dopamine, adrenergic, histamine, vasopressin and somatostatin receptor subgroups. The GPCRs were stimulated with their corresponding agonists serotonin (5HT), dopamine (DA), epinephrine (Epi), histamine (His), vasopressin (AVP), and somatostatin (SST) (Figure 15Figure 16). The GPCRs activation was tested in different heterologous cell lines to check receptor performance under different cellular backgrounds. The tested cell lines were the human osteosarcoma cell line U2OS (Figure 14), the rat pheochromocytoma cell line PC12 tetOFF (Figure 15) and the Chinese hamster ovary cell line (CHO) (Figure 16).

Significant stimulation-dependent receptor activation could be detected in U2OS cells for all serotonin receptors (Figure 14A), the dopamine receptors DRD1, DRD2, DRD4 and DRD5 (Figure 14B), the histamine receptor HRH1 (Figure 14C), the adrenergic receptors ADRA2B, ADDRA2C, ADRB2 and ADRB3 (Figure 14D), both vasopressin receptors AVPR1A and AVPR2 (Figure 14E) and for the somatostatin receptors SSTR2 and SSTR3 (Figure 14F).

In PC12 cells, with the exception of HTR1A and HTR7, all serotonin, histamine, adrenergic, vasopressin and somatostatin receptors showed significant receptor activation after stimulation (Figure 15A, C-F). For the dopamine receptors, only DRD1 showed receptor activation (Figure 15B).

In $\mathrm{CHO}$ cells, all adrenergic and vasopressin receptors showed stimulation-dependent activations (Figure 16C, D). Likewise the dopamine and somatostatin receptors with exception of DRD3 and SSTR1 showed significant stimulation-dependent signal changes (Figure 16B, E). From the serotonin receptors only for HTR5A significant receptor activation could be detected. However, for all serotonin receptors higher luciferase readings in the stimulated conditions compared to the corresponding unstimulated samples could be observed (Figure 16A).

By comparing the three cell lines U2OS, PC12 and CHO cell-type specific differences in the receptor activations were observed. The performance of each GPCR differed 
between the cellular backgrounds. All GPCR activations obtained in luciferase assays in the different cell lines are summarised in Table 6.

Table 6: Summary table of GPCR performance in luciferase assays.

Listed are all GPCRs monitored in luciferase-based split TEV assays. Significant receptor activations measured in U2OS, PC12 and $\mathrm{CHO}$ cells are indicated by a tickmark.

\begin{tabular}{lccc} 
GPCR & U2OS & PC12 & CHO \\
\hline HTR1A & $\checkmark$ & - & - \\
\hline HTR2A & $\checkmark$ & $\checkmark$ & - \\
\hline HTR4 & $\checkmark$ & $\checkmark$ & - \\
\hline HTR5A & $\checkmark$ & $\checkmark$ & $\checkmark$ \\
\hline HTR7 & $\checkmark$ & - & - \\
\hline DRD1 & $\checkmark$ & $\checkmark$ & $\checkmark$ \\
\hline DRD2 & $\checkmark$ & - & $\checkmark$ \\
\hline DRD3 & - & - & - \\
\hline DRD4 & $\checkmark$ & - & $\checkmark$ \\
\hline DRD5 & $\checkmark$ & - & $\checkmark$ \\
\hline ADRA1A & - & $\checkmark$ & $\checkmark$ \\
\hline ADRA2B & $\checkmark$ & $\checkmark$ & $\checkmark$ \\
\hline ADRA2C & $\checkmark$ & $\checkmark$ & $\checkmark$ \\
\hline ADRB2 & $\checkmark$ & $\checkmark$ & $\checkmark$ \\
\hline ADRB3 & $\checkmark$ & $\checkmark$ & $\checkmark$ \\
\hline HRH1 & $\checkmark$ & $\checkmark$ & not measured \\
\hline AVPR1A & $\checkmark$ & $\checkmark$ & $\checkmark$ \\
\hline AVPR2 & $\checkmark$ & $\checkmark$ & $\checkmark$ \\
\hline SSTR1 & - & $\checkmark$ & - \\
\hline SSTR2 & $\checkmark$ & $\checkmark$ & $\checkmark$ \\
\hline SSTR3 & $\checkmark$ & $\checkmark$ & $\checkmark$ \\
\hline
\end{tabular}


A

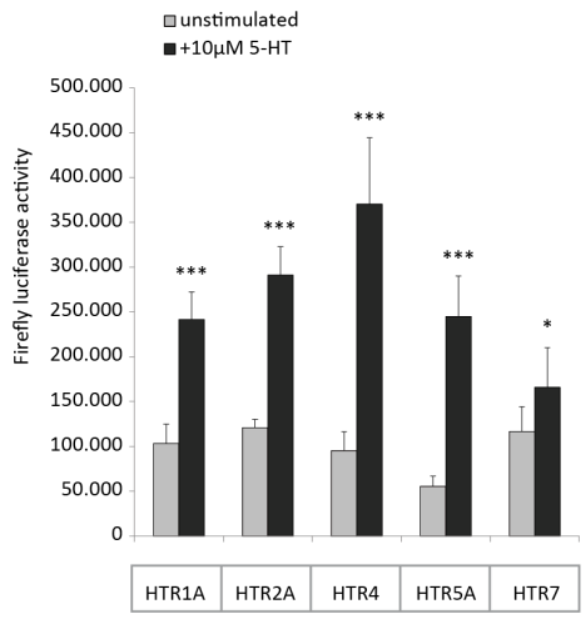

D

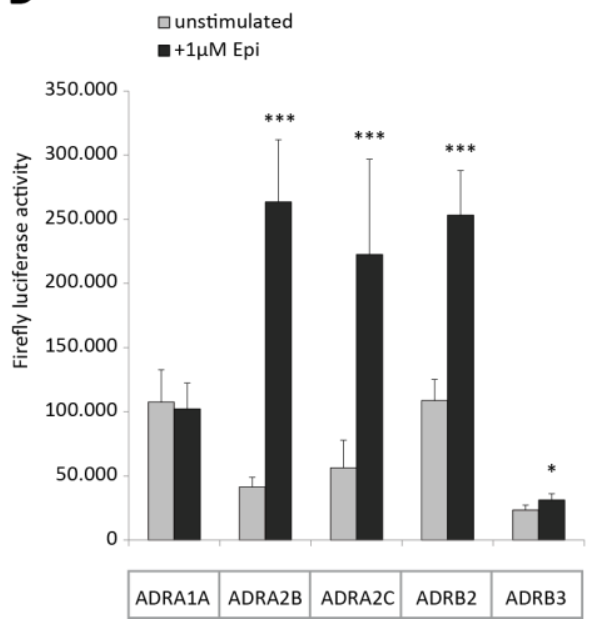

B

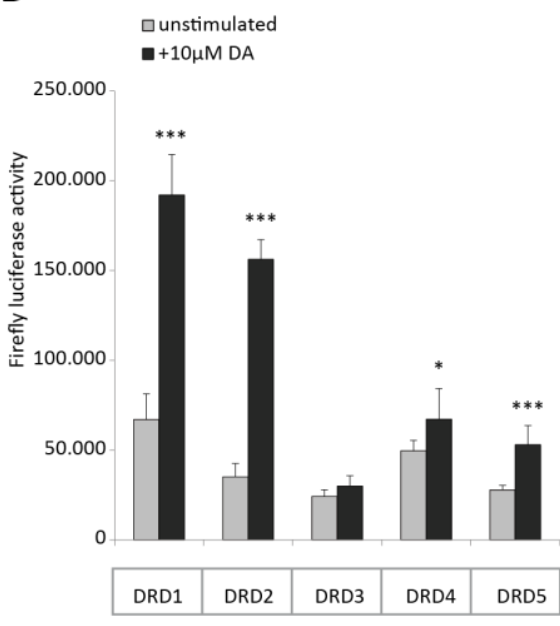

C

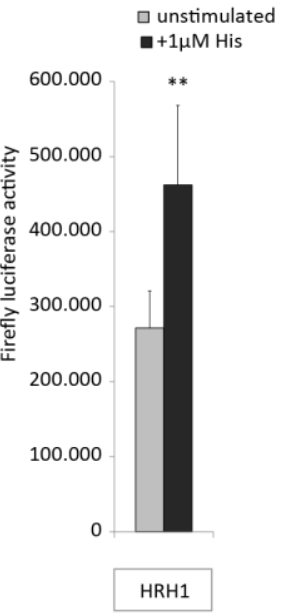

$\mathbf{E}$

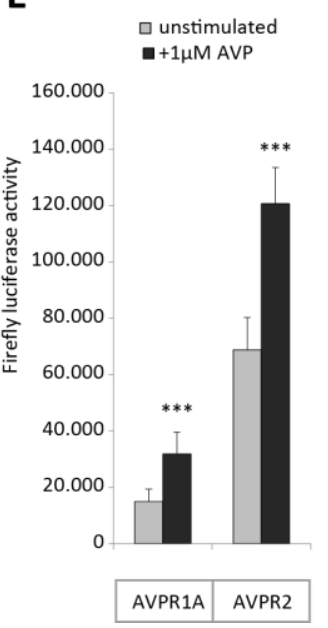

$\mathbf{F}$

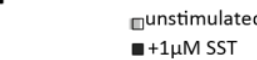

350.000

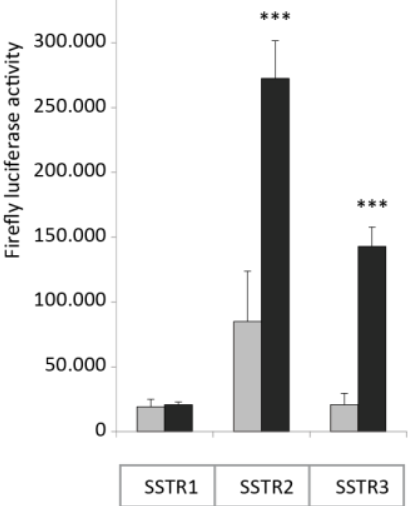

Figure 14: Split TEV luciferase assay to monitor ligand dependent GPCR activation in U2OS cells.

Serotonin (A), dopamine (B), histamine (C), adrenergic (D), vasopressin (E) and somatostatin (F) receptors were fused to V2R-NTEV-tevS-GV and transfected with $\beta$-arrestin2 $\triangle$-CTEV into U2OS cells. Cells were stimulated with agonists [5-HT, serotonin (A); DA, dopamine (B); His, histamine (C); Epi, epinephrine (D), AVP, vasopressin (E); SST, somatostatin (F)] at indicated concentrations for $6 \mathrm{~h}$. Luciferase data are given as means \pm standard deviations $(n=6)$. ${ }^{*} p \leq 0.05 ;{ }^{* *} p \leq 0.01 ;{ }^{*} p \leq 0.001$ (twosided t-test). 
A

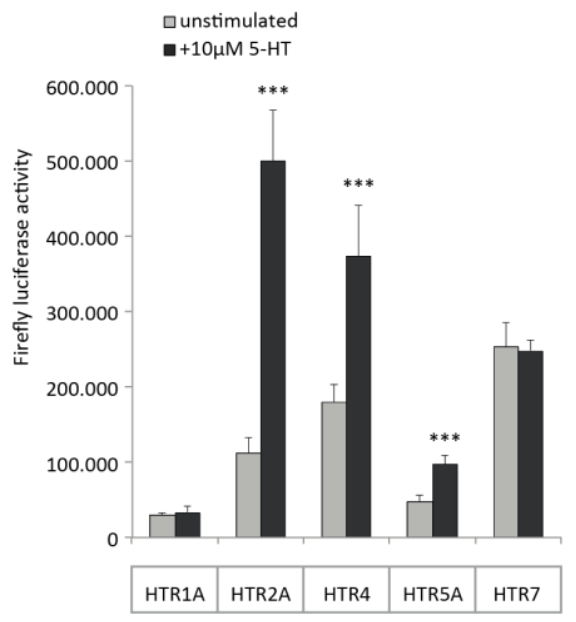

D

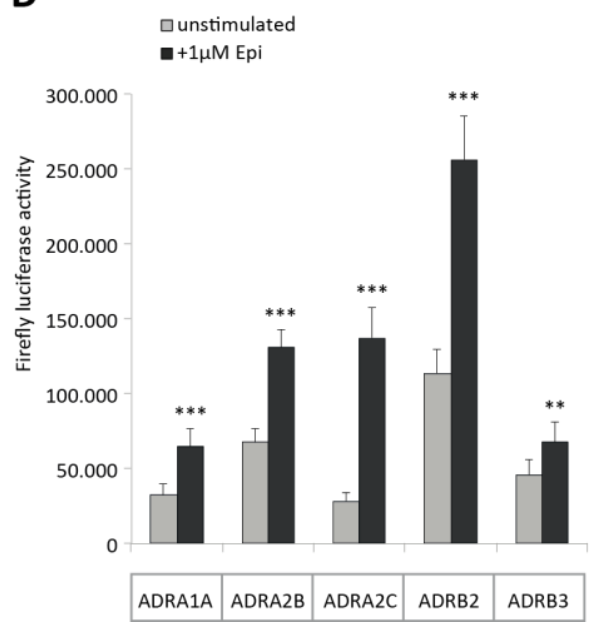

B

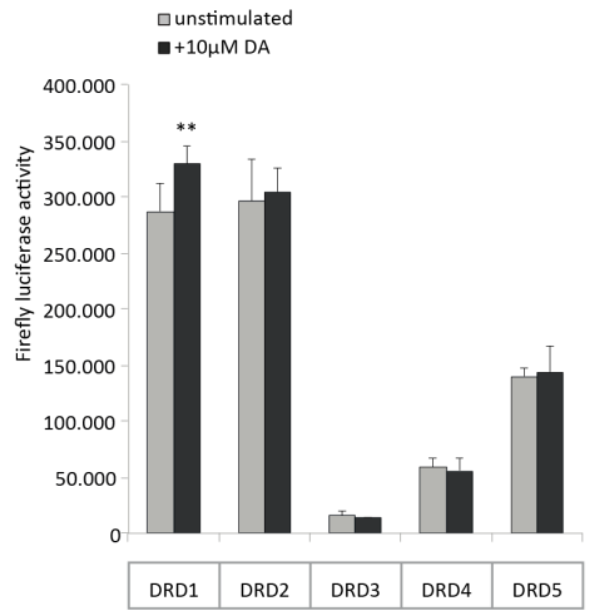

C

$\square$ unstimulated

a $+1 \mu \mathrm{M}$ His

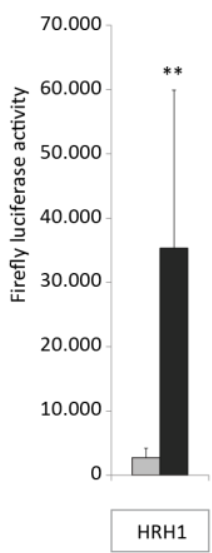

$\mathbf{E}$

$\square$ unstimulated - $+1 \mu \mathrm{M}$ AVP

$\mathbf{F}$

180.000

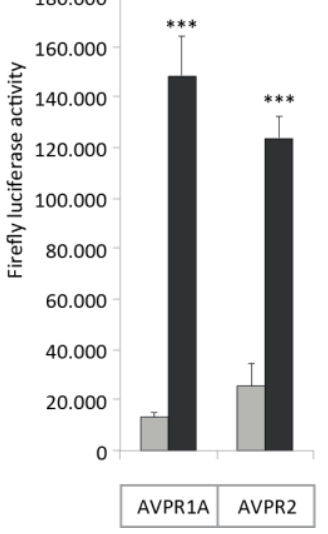

口unstimulated

- $+1 \mu \mathrm{M}$ SST

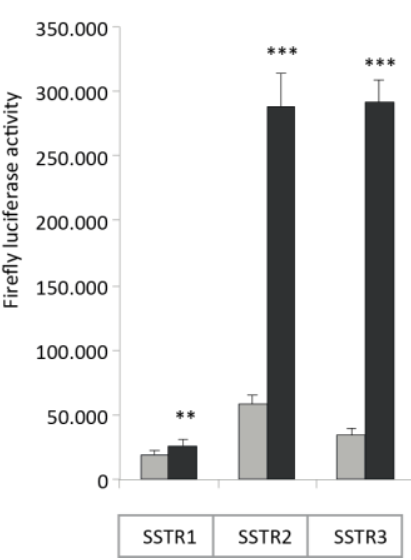

Figure 15: Split TEV luciferase assay to monitor ligand dependent GPCR activation in PC12 cells.

Serotonin (A), dopamine (B), histamine (C), adrenergic (D), vasopressin (E) and somatostatin (F) receptors were fused to V2R-NTEV-tevS-GV and transfected with $\beta$-arrestin2 $\triangle$-CTEV into PC12tetOFF cells. Cells were stimulated with agonists [5-HT, serotonin (A); DA, dopamine (B); His, histamine (C); Epi, epinephrine (D), AVP, vasopressin (E); SST, somatostatin (F)] at indicated concentrations for $6 \mathrm{~h}$. Luciferase data are given as means \pm standard deviations $(n=6)$. ${ }^{*} p \leq 0.05 ;{ }^{* *} p \leq 0.01 ;{ }^{*} p \leq 0.001$ (twosided t-test). 
A

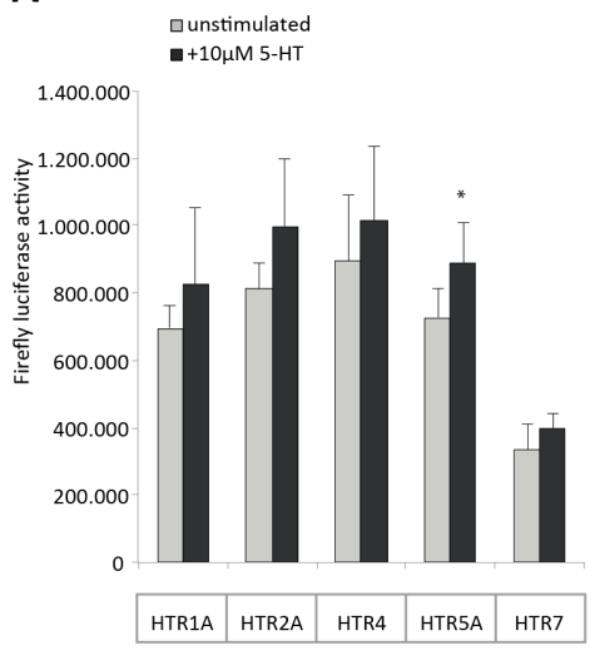

C

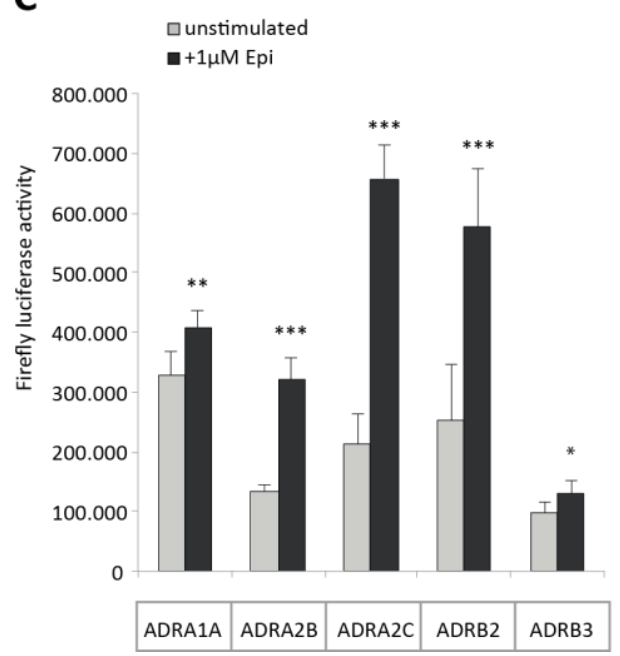

B

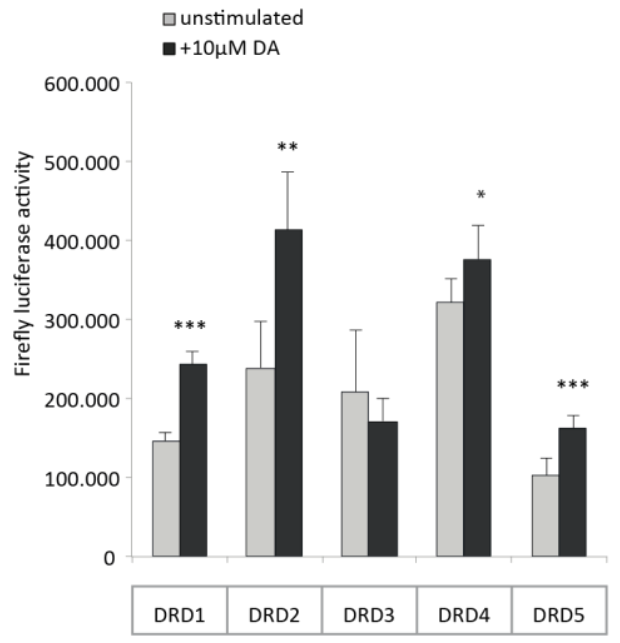

D

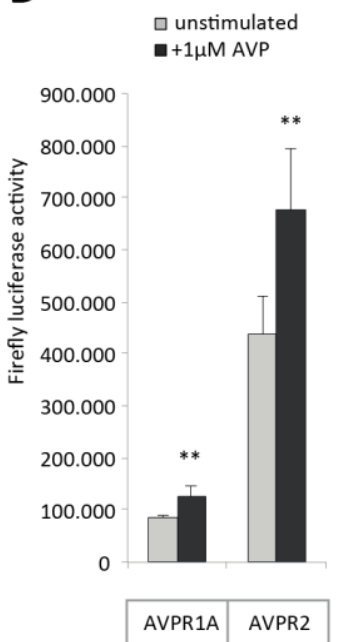

$\mathbf{E}$

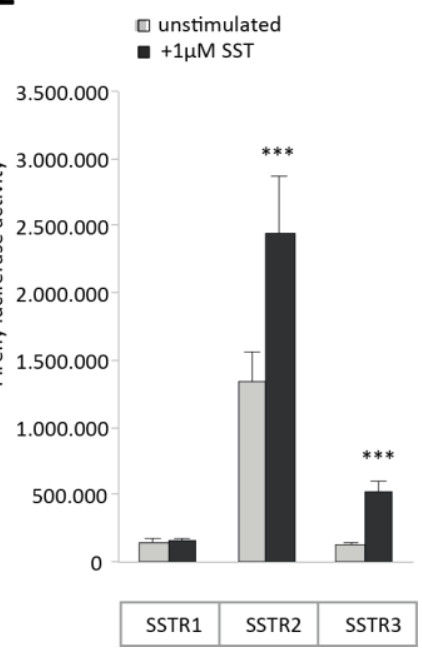

Figure 16: Split TEV luciferase assay to monitor ligand dependent GPCR activation in CHO cells.

Serotonin (A), dopamine (B), adrenergic $(C)$, vasopressin $(D)$ and somatostatin $(E)$ receptors were fused to V2R-NTEV-tevS-GV and transfected with $\beta$-arrestin2 $\triangle$-CTEV into $C H O$ cells. Cells were stimulated with agonists [5-HT, serotonin (A); DA, dopamine (B); Epi, epinephrine (C), AVP, vasopressin (D); SST, somatostatin (E)] at indicated concentrations for $6 \mathrm{~h}$. Luciferase data are given as means \pm standard deviations ( $n=6) .{ }^{*} p \leq 0.05 ;{ }^{* *} p \leq 0.01 ;{ }^{*} p \leq 0.001$ (two-sided t-test). 


\subsubsection{Dose-dependent GPCR activation and inhibition}

An important part in studying stimulation-dependent receptor activation is the description of the dose-response relationship between ligand concentration (dose) and receptor response. At a given ligand concentration threshold, the receptor response reaches an activation plateau and cannot be increased any more by addition of higher ligand levels. This relationship is commonly depicted as a dose response curve by plotting the receptor response to the logarithm of the ligand concentration.

A dose-response curve provides information about the potency of ligands by determining $\mathrm{EC}_{50}$ or $\mathrm{IC}_{50}$ values. The $\mathrm{EC}_{50}$ value (half maximal effective concentration) is the concentration of an agonist required to produce $50 \%$ of receptor response. The $I_{50}$ value (half maximal inhibiting concentration) is the concentration of an antagonist required for $50 \%$ inhibition of the maximal receptor response of an agonist in vitro.

The applicability of split TEV assays to monitor dose-dependent GPCR activation was tested for a selection of GPCRs in luciferase assays. Because of the observed cell linedependent receptor performance, the assays were carried out in either PC12 or U2OS cells.

The serotonin receptors HTR2A and HTR4 showed in PC12 cells a dose-dependent activation by stimulation with serotonin with an $\mathrm{EC}_{50}$ of $0.51 \mu \mathrm{M}$ and $0.63 \mu \mathrm{M}$ (Figure 17A, B). The dose-dependent stimulation of the dopamine receptors DRD1 and DRD2 with dopamine resulted in an $\mathrm{EC}_{50}$ of $0.8 \mu \mathrm{M}$ for DRD1 and $3 \mu \mathrm{M}$ for DRD2 in U2OS cells (Figure 17C, D). Both adrenergic receptors ADRA2B and ADRB2 showed a dosedependent activation induced by isoproterenol, $A D R A 1 A$ with an $E_{50}$ of $0.41 \mu \mathrm{M}$ in PC12 cells and ADRB2 with $\mathrm{EC}_{50}$ of $0.94 \mu \mathrm{M}$ in U2OS cells (Figure 17E, F). 
A

HTR2A

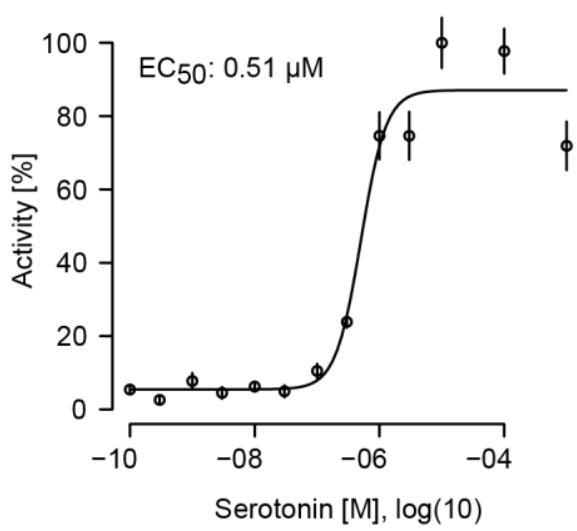

C

DRD1

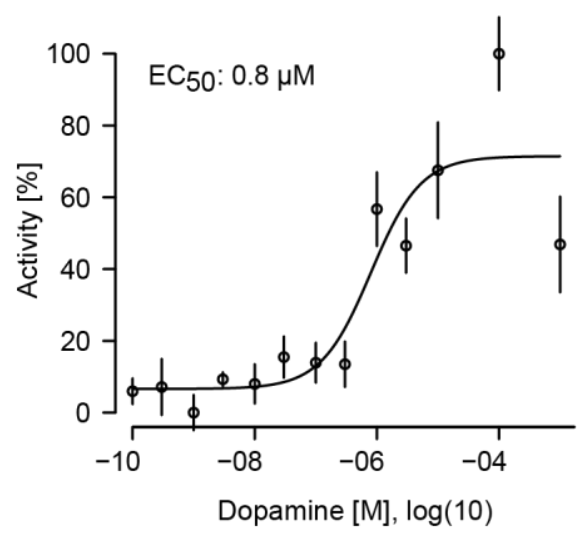

E

ADRA2B

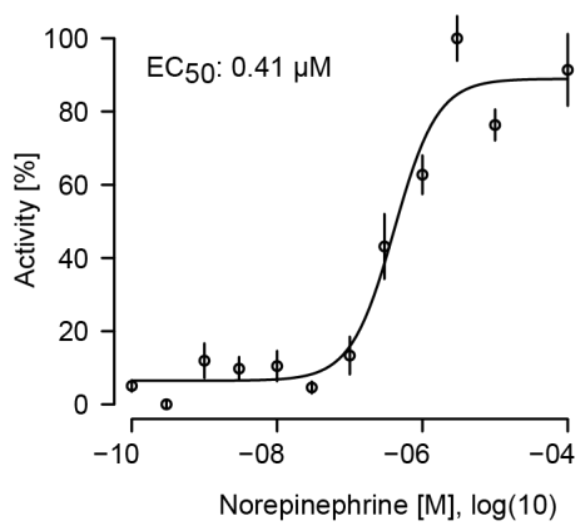

B

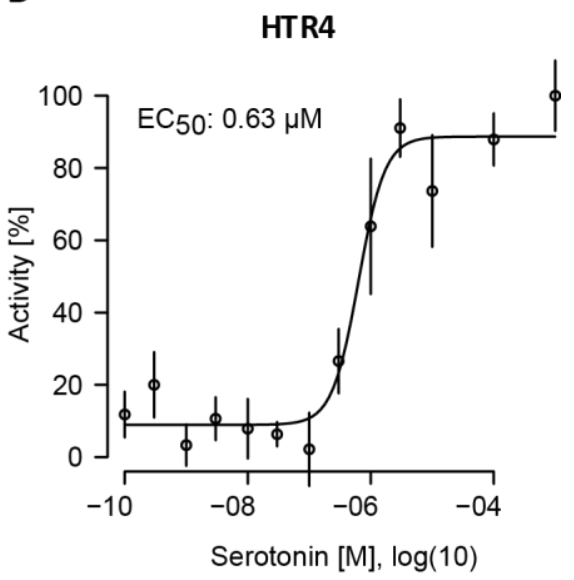

D

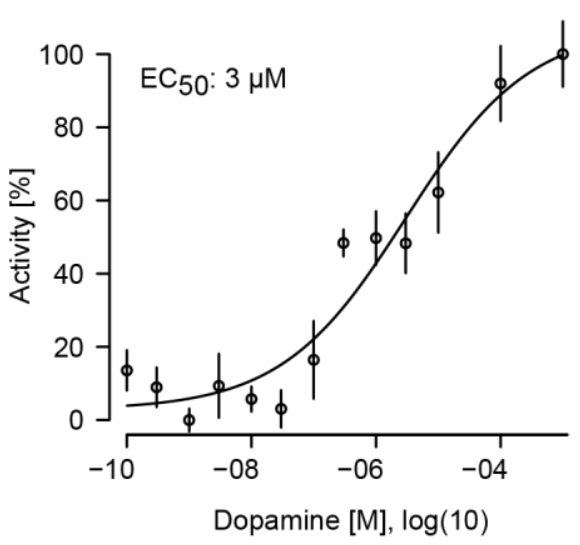

F

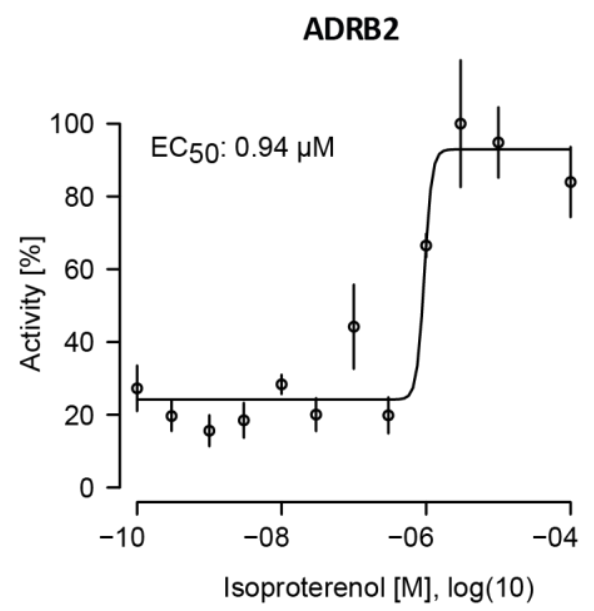

Figure 17: Dose-dependent GPCR activation by agonists in luciferase assays.

Dose-dependent GPCR activations were measured in luciferase assays using GPCR-V2R-NTEV-tevS-GV and Barr2 $\triangle$-CTEV fusion constructs. Assays with HTR2A (A), HTR4 (B) and ADRA2B (E) were performed in PC12 cells, assays with DRD1 (C), DRD2 (D) and ADRB2 (F) were carried out in U2OS cells. Cells were stimulated with agonists for $6 \mathrm{~h}$. Dose-response curves were fitted with $\mathrm{R}$ ('drc' package). Luciferase data are given as means $\pm \operatorname{SEM}(n=6)$. 
The applicability of split TEV assays to monitor the effects of antagonists to inhibit GPCR responses was tested by treatment of the serotonin receptor HTR2A with the two neuroleptics asenapine and paliperidone. The antagonist assay was done in PC12 cells. The receptor was pre-incubated with the particular antagonist for $1 \mathrm{~h}$ before adding the agonist serotonin at its $\mathrm{EC}_{50}$ concentration (Figure 17A) of $0.7 \mu \mathrm{M}$. Asenapine showed a dose-dependent inhibition of the serotonin induced HTR2A response with an $\mathrm{IC}_{50}$ of $0.44 \mathrm{nM}$ (Figure 18A). For paliperidone a dose-dependent HTR2A inhibition could be monitored with an $\mathrm{IC}_{50}$ of $1.35 \mathrm{nM}$ (Figure 18B).

A

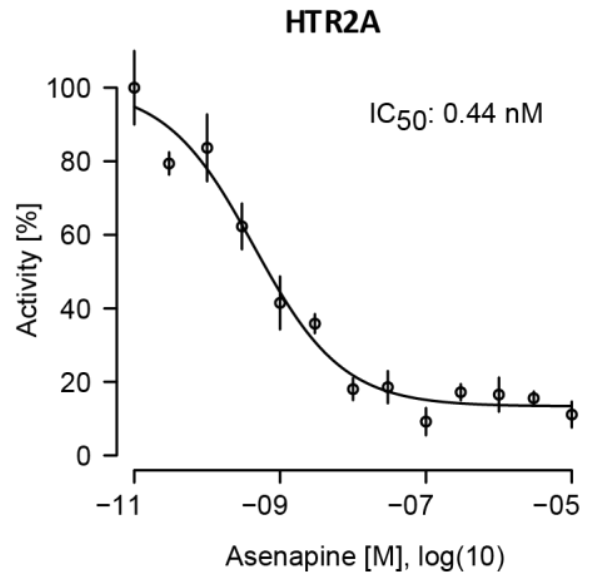

B

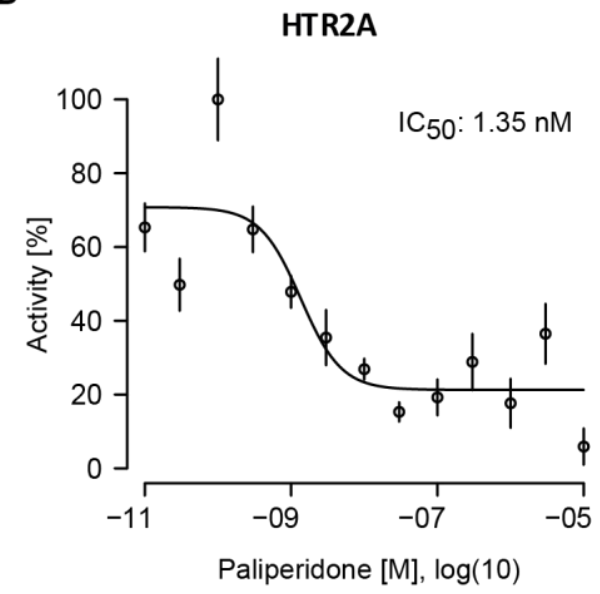

Figure 18: Dose-dependent GPCR inhibition by antagonists in luciferase assays.

Dose-dependent HTR2A inhibition by the antagonists asenapine and paliperidone was tested in PC12 cells using luciferase read out. Cells were pre-treated with either asenapine (A) or paliperidone (B) for $1 \mathrm{~h}$ and subsequently stimulated with $0.7 \mu \mathrm{M}$ serotonin for additional $6 \mathrm{~h}$. Dose-response curves were fitted with $R$ ('drc' package). Luciferase data are given as means $\pm \operatorname{SEM}(n=6)$. 


\subsection{EXT-based GPCR activation assays}

\subsubsection{Principle of multiplexed GPCR activation EXT assays}

Receptor ligands often have the property to act on more than one specific receptor but on a subset of receptors as ligands may bind to several receptors. To determine the mode of action of ligands on multiple receptors single luciferase assays becomes inconvenient due to their limitation of ascertainable data points. A novel method to monitor the ligand-dependent receptor activation of multiple GPCRs simultaneously are EXT based GPCR assays. The principle of such multiplexed GPCR activation assays is the combination of the split TEV technology with EXT barcode reporters (EXTassays). Instead of using reporter proteins such as firefly luciferase as read out, EXT reporters produce unique RNA sequences that can be analysed in a pool. This feature allows to assign each EXT reporter to a unique cellular assay, in this case to a particular GPCR activity assay, and analyse a cell population expressing multiple EXT reporters and GPCRs at the same time under the same experimental conditions.

The general experimental set up of multiplexed GPCR activation EXT assays is composed of separate in-solution transfections of single GPCR split TEV components (GPCR-V2R-NTEV-tevS-GV and $\beta$-arrestin2 $\Delta$-CTEV) and EXT reporters. Three unique 10xUAS-EXT reporters were assigned to each GPCR (assay EXTs). In addition, three to six 10xUAS-EXT reporters were transfected separately without any GPCR as control for sample handling and unwanted condition effects and serve as internal calibrators (iCals). Two hours after transfection all cell populations were pooled together, mixed and plated out evenly distributed (Figure 19A). The cells were stimulated according to the previously described experimental workflow for agonist or drug assays (see chapter 5.1.4). After cell lysis the RNA from each sample was isolated and converted into cDNA by reverse transcription. To control the next steps of amplification and sequencing, additional EXT reporter plasmids were added as external calibrators (eCals) to the cDNA. As eCal reporters 5xUAS-TK-EXT constructs were used encoding a 5 times clustered UAS element followed by the herpes simplex virus thymidine kinase promoter (TK) and unique EXT sequences. The assay EXT sequences as well as the iCal and eCal EXTs were then amplified by a decoding PCR. Afterwards each sample was labelled with a unique sequence barcode by an additional code PCR. This enables the 
mixing of all samples and sequencing of the pool by next-generation-sequencing using the Ion Torrent Personal Genome Machine (PGM) (Figure 19B). Detailed description of the sample preparation and sequencing procedure is given in the methods part, chapters 4.3 and 4.4 .

A

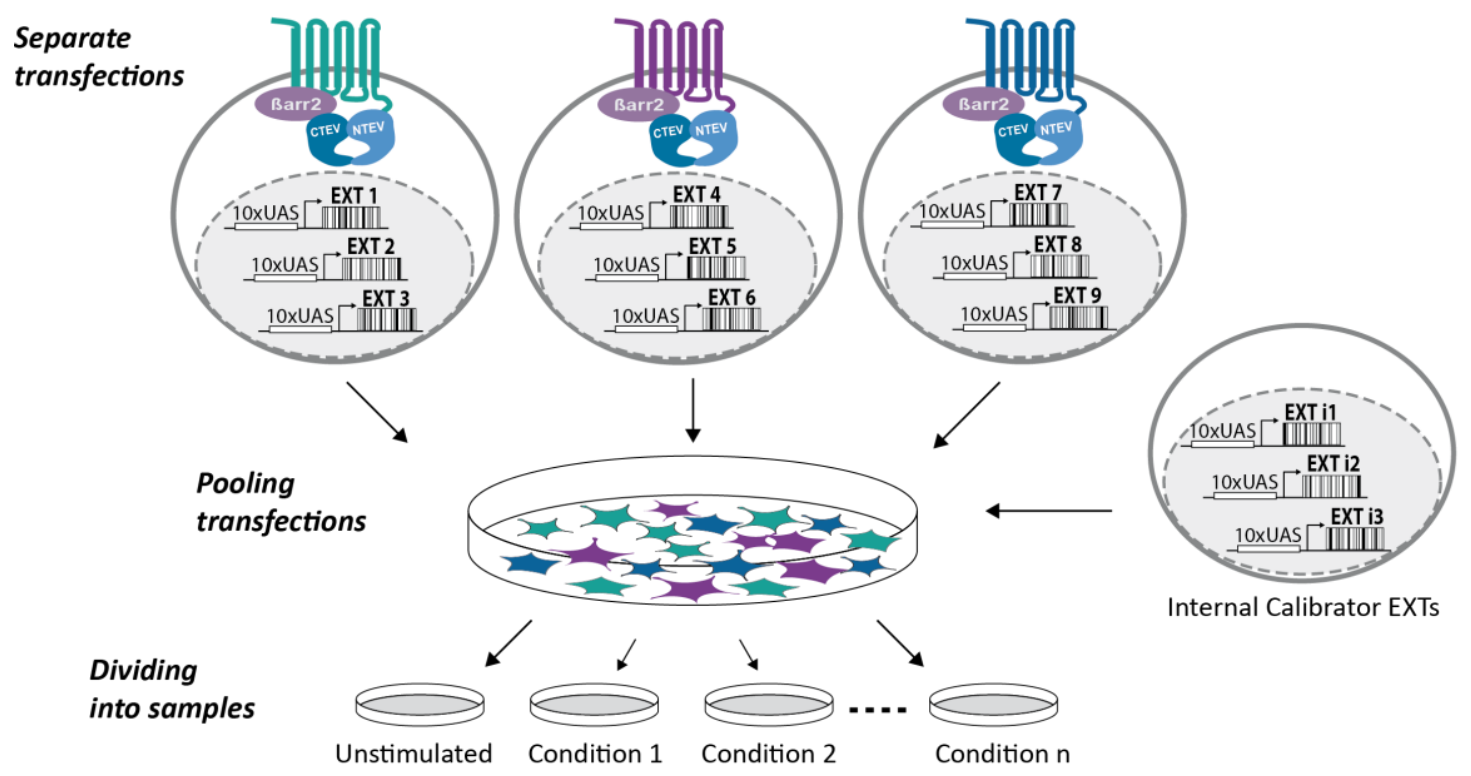

B

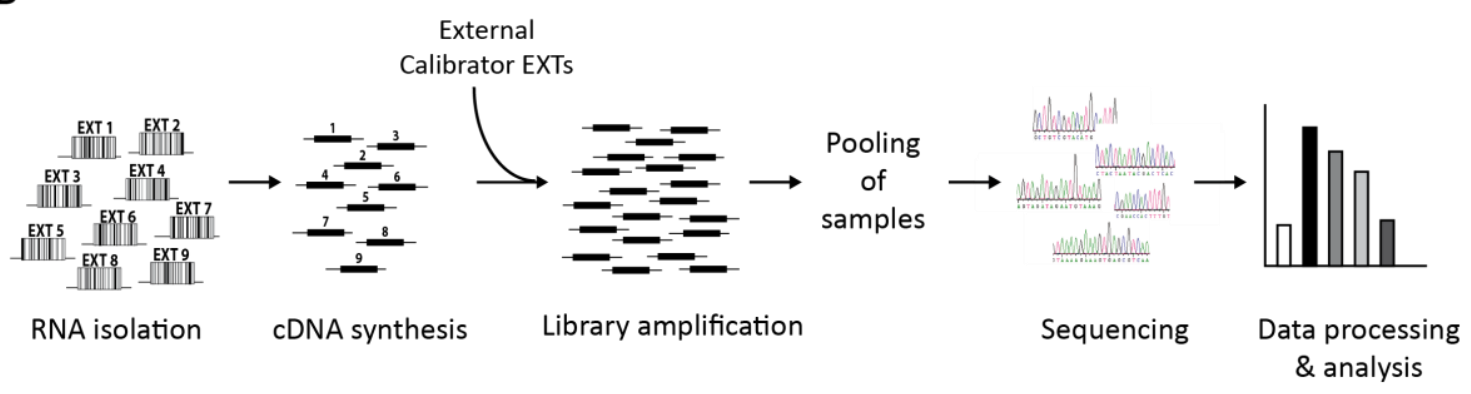

Figure 19: Schematic design of multiplexed EXT-based GPCR activation assays.

(A) For each GPCR a separate in-solution transfection of receptor and $\beta$-arrestin2 $\triangle$ split TEV fusion plasmids and 3 unique 10xUAS-EXT reporters was done. Additional 10xUAS-EXT reporters were transfected as internal calibrators. After 2 hours all transfections were pooled, divided into samples and plated out. Samples were treated depending on the assay. (B) Processing of samples after cell lysis. From each sample the total RNA was isolated and converted into CDNA. After adding of 5xUAS-TK-EXTs as external calibrators EXT sequences were PCR amplified and barcoded. Afterwards samples were pooled and sequenced by next generation sequencing followed by raw data processing and analysis. 


\subsubsection{Processing of sequencing data}

To analyse the biological output of the EXT assays the raw sequencing data obtained from the Ion Torrent sequencer needed to undergo normalisation, calibration and standardisation steps (Figure 20). Each GPCR EXT assay is composed of a defined number of samples, with each sample encoding a specific treatment condition. To balance differences between the samples occurred during the sequencing process the total read number of each sample was calculated and normalized to the highest total read number (Figure 21). As low read numbers are instable, all samples with total reads under a certain threshold were excluded from the experiment. The threshold was commonly set to the $20 \%$ value of the highest total read number. To control differences between samples that can occur at the level of cell handling and the later sample preparations including cDNA synthesis and library amplification calibrator EXTs were included into the experiment at the transfection level (internal calibrators, iCals) and before the library amplification (external calibrators, eCals). The eCals are also used to compensate so called "read eating"- effects. Within a sample highly expressed EXTs have an advantage during the library amplification PCRs and the sequencing reaction which leads to the suppression of effects of lower expressed EXTs. These "read eating"- effects can be detected in samples with low read numbers of the eCals (Figure 22A). To balance these effects all reads were calibrated to normalisation factor based on the means of eCal reads of each sample (Figure 22B). Following to normalisation and calibration steps, the biological sample replicates and the three EXTs assigned to the GPCRs were consolidated and the resulting samples standardised to a reference sample. In GPCR activation EXT assays, all samples with an applied stimulus were standardised to the unstimulated control sample to calculate the foldchanges of receptor activation.

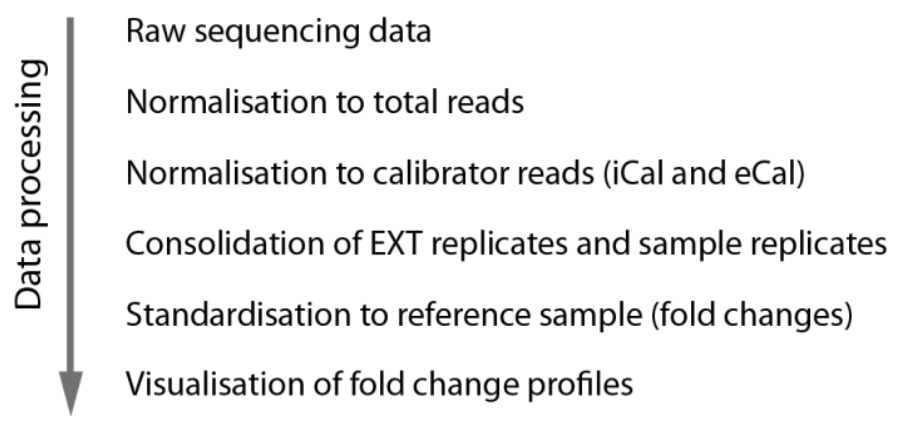

Figure 20: Data processing workflow of GPCR activation EXT assays. 


\subsubsection{GPCR activation profiles in U2OS cells}

The first EXT-based GPCR assay was performed to assess the ligand-dependent activation of 20 GPCRs (Table 5, without HRH1) simultaneously in one experiment. All receptors were stimulated with the agonists serotonin (5-HT), dopamine (DA), epinephrine (Epi), vasopressin (AVP), and somatostatin (SST) separately in a concentration range of $100 \mathrm{pM}$ to $100 \mu \mathrm{M}$. Additionally to the stimulation with the single compounds, a compound-mixture (Mix) of all five agonists was used. The experiment was carried out in human osteosarcoma cell line U2OS. For each GPCR three unique EXT reporters were assigned and each condition was done in two replicates. After stimulation for $6 \mathrm{~h}$, the EXT RNA from all samples was extracted and processed as previously described (Figure 19B). The sequencing raw data were normalised to total read numbers (Figure 21) and to external and internal calibrator EXTs (Figure 22). Fold changes of receptor activation are based on the average of normalised EXT signals in reference to unstimulated conditions.

A

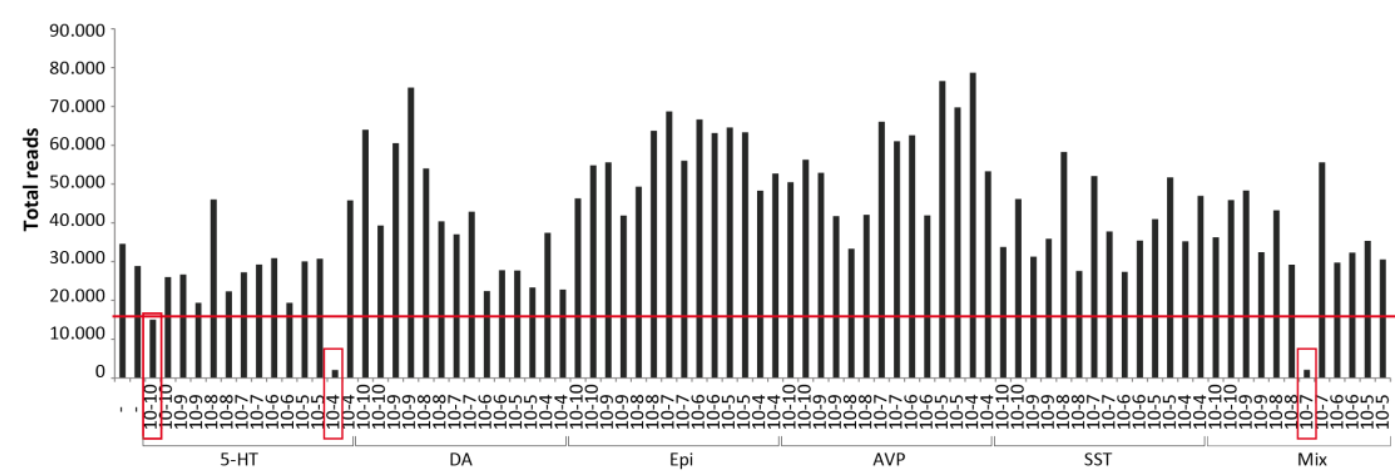

B

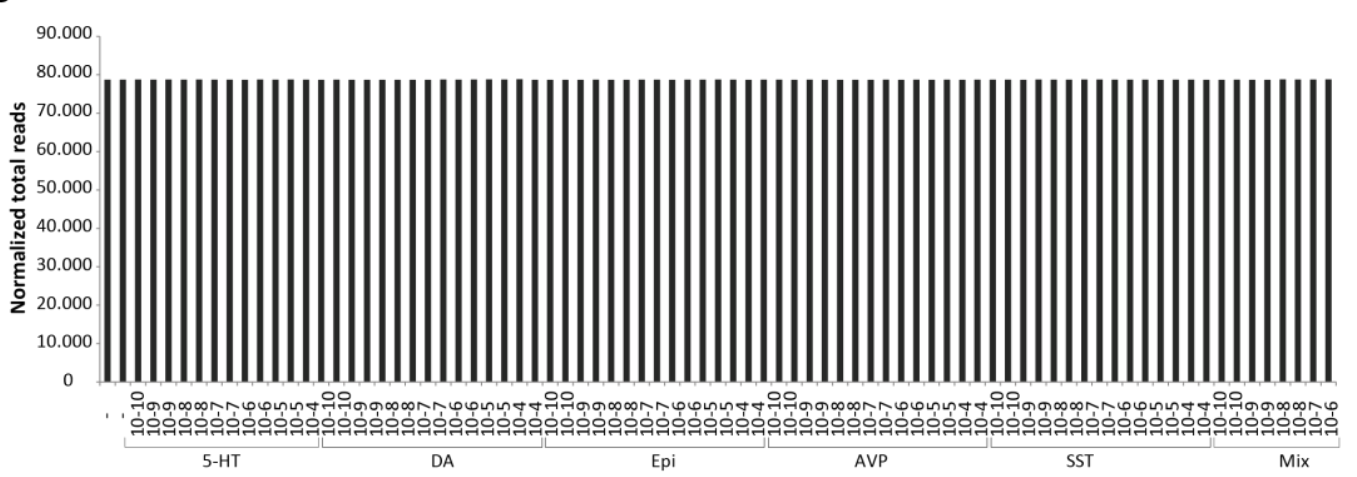

Figure 21: Normalisation of sequencing raw data to total reads.

(A) Total reads per sample of raw sequencing data. The red line marks the threshold of the $20 \%$ value of the highest total read number. Three samples with a total read value below the threshold were excluded from further analysis (samples are marked by red boxes). (B) Total reads per sample after normalisation. 
A

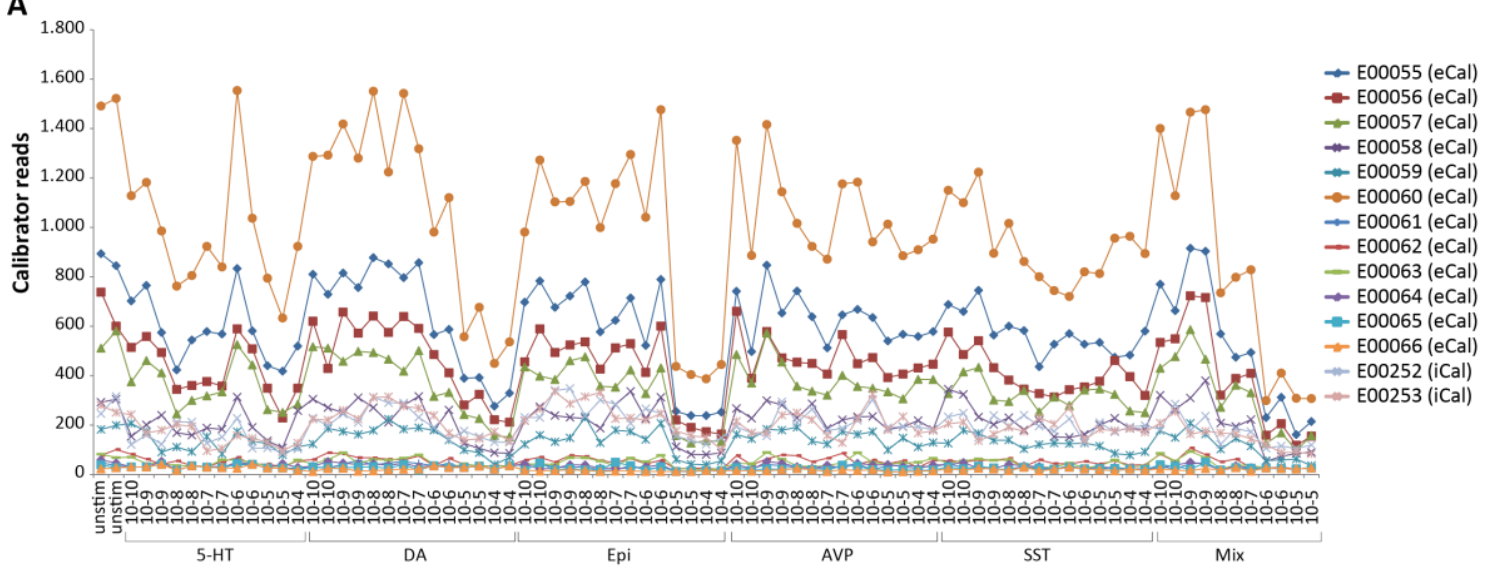

B

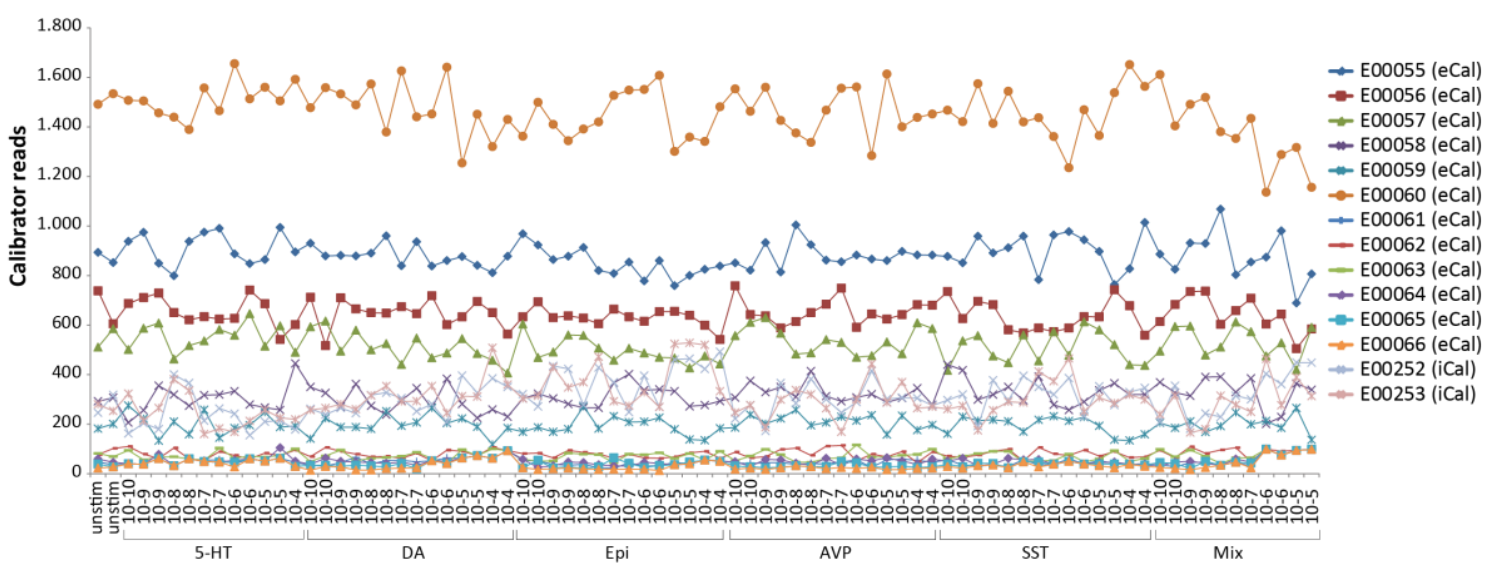

Figure 22: Normalisation to calibrator EXTs.

(A) Reads of external (eCal) and internal (iCal) calibrator EXTs after normalisation to total reads. Normalisation factor was calculated from the highest mean of samples. (B) Calibrator EXT reads after application of normalization factor.

The activation profiles produced for each GPCR revealed a dose-dependent receptor activation induced by the corresponding agonist (Figure 23). The five serotonin receptors (HTR1A, HTR2A, HTR4, HTR5 and HTR7) were activated by serotonin up to 2-5 folds at the higher concentrations. Likewise vasopressin receptors AVPR1A and AVPR2 were induced by vasopressin. The induction rates of the somatostatin receptors by somatostatin could be detected for SSTR1 up to 4-fold and for SSTR2 and SSTR3 up to 9-10 fold. For the dopamine and the adrenergic receptors, a "cross"-stimulation could be observed. Both receptor groups showed elevated signals induced by dopamine and epinephrine with maximal induction rates from 2-fold for DRD1, DRD3 and ADRB3 up to $10-$ and 13 -fold for ADRA2B and ADRA2C. The dopamine receptor 
DRD2 showed also an activation of 3-5 folds at 10-100 $\mu \mathrm{M}$ serotonin concentrations. The stimulation conditions with the mixture of agonists showed dose-dependent activation signals for all GPCRs, except for HTR7 and ADRB3, which were similar in their intensities to the observed signals in the corresponding single agonist conditions.

Serotonin Dopamine Epinephrine Vasopressin Somatostatin Mix

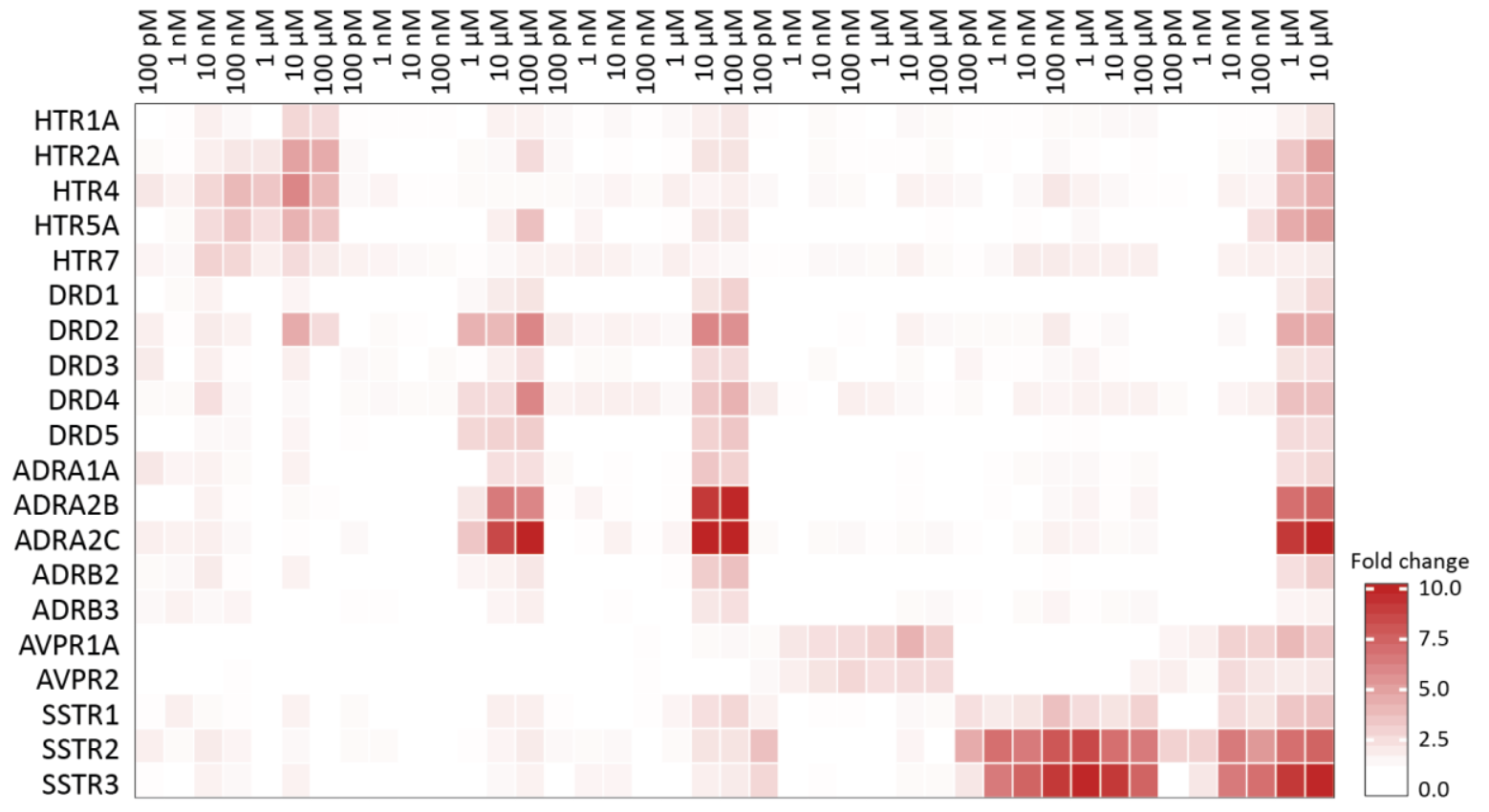

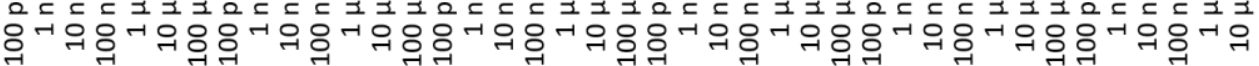

Figure 23: Profiles of GPCR activations in U2OS cells.

Heatmap of multiplexed EXT-based measurement of GPCR activations. Plotted are fold changes of receptor activation in reference to unstimulated condition of 20 different GPCRs in U2OS cells. All GPCRs were treated with the single compounds serotonin, dopamine, epinephrine, vasopressin and somatostatin and a mixture (Mix) of the five compounds at different concentrations. To visualize lower effects the colour scale is limited to 10 -fold changes.

In summary, the results suggest that the used agonists, which are classical endogenous neurotransmitters and hormones, are specific in their activation to particular GPCR subgroups, with the exception of dopamine and epinephrine causing cross-talk activities on the two subgroups of dopaminergic and adrenergic receptors. 


\subsubsection{GPCR activation profiles in PC12 cells}

In order to test the assay performance in another cellular background, the activation profiles were monitored in the rat pheochromocytoma PC12 tetOFF cell line. 19 GPCRs were stimulated with serotonin (5-HT), dopamine (DA), epinephrine (Epi), vasopressin (AVP), and somatostatin (SST) separately and the compound-mix (Mix) of all five agonists for $6 \mathrm{~h}$ (Figure 24).

\section{Serotonin Dopamine Epinephrine Vasopressin Somatostatin Mix}

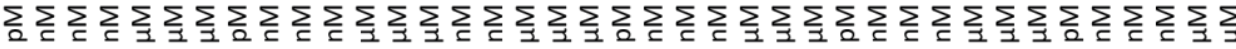

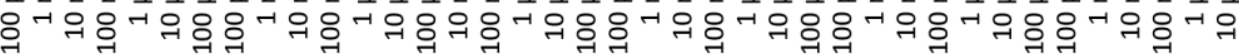

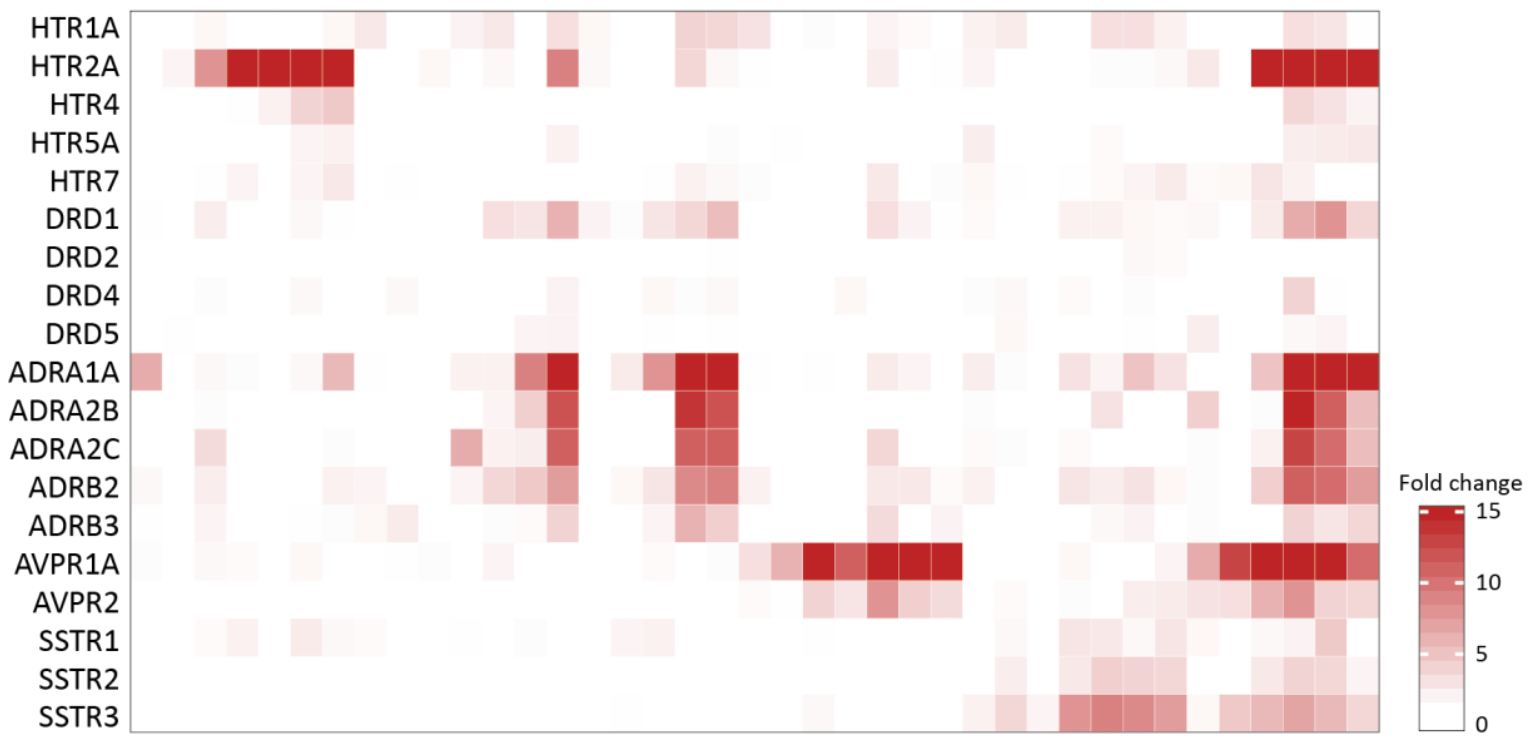

Figure 24: Profiles of GPCR activations in PC12 tetOFF cells.

Heatmap of multiplexed GPCR activation EXT assay. Plotted are fold changes of receptor activation in reference to unstimulated condition of 20 different GPCRs in PC12 tetOFF cells. Treatment conditions at different concentrations were the single compounds serotonin, dopamine, epinephrine, vasopressin and somatostatin and a mixture (Mix) of the five compounds. To visualize lower effects the colour scale is limited to 15 -fold changes.

In contrast to the activation profiles observed in U2OS cells in PC12 cells not all GPCRs showed a dose-dependent activation stimulated with the corresponding endogenous agonist (Figure 24). Within the group of serotonin receptors HTR1A showed no response to serotonin and HTR5A and HTR7 only weak activation of 2-fold while for HTR2A an up to 90 -fold increase in activity by serotonin could be observed. From the dopamine receptors for DRD1 dose-dependent receptor activation could be detected 
whereas DRD2 showed no response to any stimulation and DRD4 and DRD5 only a 2 -fold activation at the highest concentration of $100 \mu \mathrm{M}$. As already in the previous experiment observed, the adrenergic receptors as well as the dopamine receptor DRD1 showed "cross"-stimulated responses to the two agonists dopamine and epinephrine. The highest activation was detected for the $\alpha$-adrenergic receptor ADRA1A with 315-fold at $100 \mu \mathrm{M}$ epinephrine. Altogether the assay performed in PC12 cells showed more background signals of "unspecific" activities compared with the assay performance in U2OS cells.

\subsubsection{Profiles of antipsychotic drug effects on GPCR activation}

The next step was the application of the multiplexed GPCR EXT assay approach to measure the effects of GPCR targeting drugs on the receptor activation.

Here, 19 different GPCRs were treated with the three FDA-approved drugs aripiprazole, paliperidone and propranolol, and the antagonist UNC0006 (Figure 25). Aripiprazole and paliperidone are commonly used atypical antipsychotic drugs. Paliperidone is the active metabolite of the older atypical antipsychotic risperidone and acts in the classical mode of action of antipsychotics as DRD2 and HTR2A antagonist. In addition, paliperidone has also binding affinities to $\alpha$-adrenergic and the histamine receptor HRH1 (Corena-McLeod, 2015; Mauri et al., 2014). In contrast, aripiprazole is controversially described either as DRD2 partial agonist or functionally selective drug acting cell type dependent as DRD2 agonist, partial agonist or even as antagonist (Lawler et al., 1999; Shapiro et al., 2003). UNC0006 is a functionally selective DRD2 ligand and aripiprazole analogue generated by changing the cyclic amines of the aripiprazole scaffold. The compound was found to be a $\beta$-arrestin-biased DRD2 partial agonist, which did not induce $G_{i}$ protein-dependent signalling, but pertained atypical antipsychotic drug-like activities in vivo (Allen et al., 2011). Propranolol is a non-selective beta blocker acting as antagonist on $\beta$-adrenergic receptors. It is mainly used in the treatment of cardiovascular diseases like high blood pressure. Further, it is also used in the prevention and treatment of migraine and anxiety (Baker, 2005). 
A<smiles>O=C1CCc2ccc(OCCCCN3CCN(c4cccc(Cl)c4Cl)CC3)cc2N1</smiles>

C<smiles>Cc1nc2n(c(=O)c1CCN1CCC(c3noc4cc(F)ccc34)CC1)CCCC2O</smiles>

Paliperidone<smiles>O=C1CCc2ccc(OCCCCN3CCCN(c4cccc(Cl)c4Cl)CC3)cc2N1</smiles>

D<smiles>CC(C)NCC(O)COc1cccc2ccccc12</smiles>

Propranolol

Figure 25: Structure of GPCR drugs.

Chemical 2D structures of: the antipsychotic drugs (A) aripiprazole and (C) paliperidone; (B) UNC0006, an analog of aripiprazole, differing in the left-hand side cyclic amines of the aripiprazole scaffold; (D) the beta blocker propranolol.

For the 19 GPCRs, separate transfections were done each comprising the constructs encoding for one GPCR and three unique 10xUAS-EXT reporters. After transfection the cells were mixed and plated out for the different stimulation conditions. Within the experiment the GPCRs were stimulated for $6 \mathrm{~h}$ under two different parameters. To monitor potential agonistic effects, GPCRs were treated with the particular drugs alone. To measure antagonistic effects GPCRs were treated with the specific drug together with the corresponding agonist. The agonists were applied after $1 \mathrm{~h}$ preincubation with the particular drugs based on the previous experiments within an agonist-mix of $1 \mu \mathrm{M}$ serotonin, dopamine, epinephrine, histamine, vasopressin, and somatostatin. Each drug was applied in several concentrations from 100 pM to $1 \mu \mathrm{M}$, each in two replicates. Thus, for the two stimulation parameters the obtained signals either result from direct drug-induced receptor activation (Figure 26A), indicating the drug as agonist, or from drug-induced inhibition of agonist-mediated receptor activation (Figure 26B), specifying the drug as antagonist. Taken together, differences in the activation profiles of individual GPCRs could be detected for these drugs (Figure 26). Effects of drug-mediated receptor activation and inhibition are highlighted in black squares in Figure 26. The calculated dose-response curves are shown in Figure 27. 
Agonistic effects could be detected on the serotonin receptor HTR2A and the dopamine receptor DRD2 induced by aripiprazole and the analogue UNC0006. The effects on HT2A are dose-dependent by aripiprazole as well as by UNC0006 (Figure 27A), but with lower maximal signals compared to the signal induced by the agonistmix. In contrast DRD2 showed only a dose-dependence for UNC0006 whereas at all concentrations of aripiprazole the activation level is as high as with the agonist-mix (Figure 27B). On the other hand both drugs showed dose-dependent receptor inhibition of the histamine receptor HRH1 (Figure 27C, D). For paliperidone and propranolol only antagonistic drug effects of receptor inhibition were detected. Paliperidone showed dose-dependent inhibition of receptor activity on multiple GPCRs, the serotonin receptor HTR2A (Figure 27E), the dopamine receptor DRD2 (Figure 27F), all three $\alpha$-adrenergic receptors ADRA1A, ADRA2B and ADRA2C (Figure 27G-I) and the histamine receptor HRH1 (Figure 27J). The treatment with propranolol showed the described beta blocker specific receptor inhibition on the $\beta$-adrenergic receptor ADRB2 (Figure 27L) and additionally an inhibition of the dopamine receptor DRD2 (Figure 27K). Except of the inhibition of the dopamine receptor DRD2 by propranolol for all observed effects binding affinities are described in the literature (Corena-McLeod, 2015; Lawler et al., 1999; Mauri et al., 2014; Shapiro et al., 2003). 
A

Paliperidone

Aripiprazole

UNC0006

Propranolol
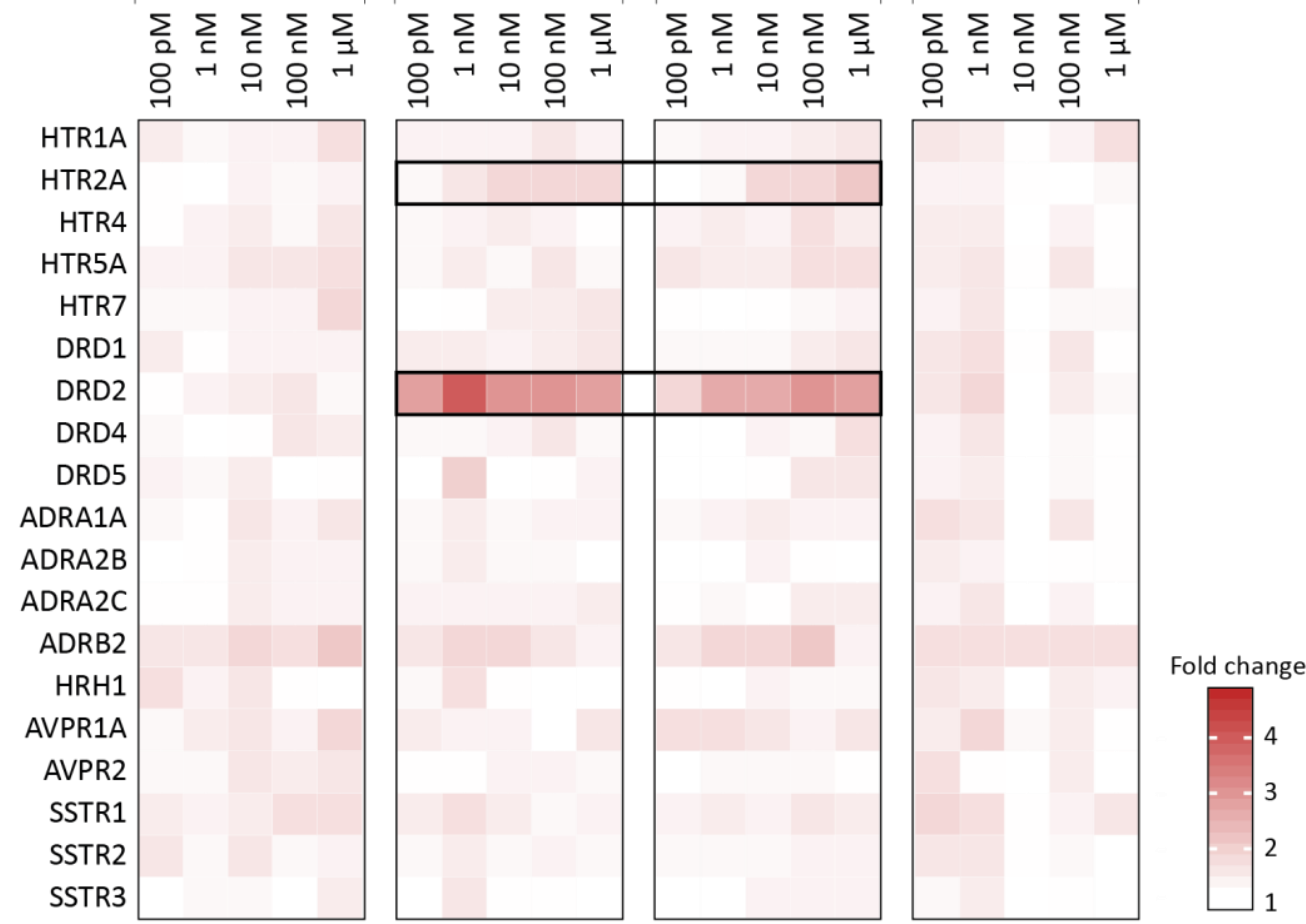

B + Agonist-Mix

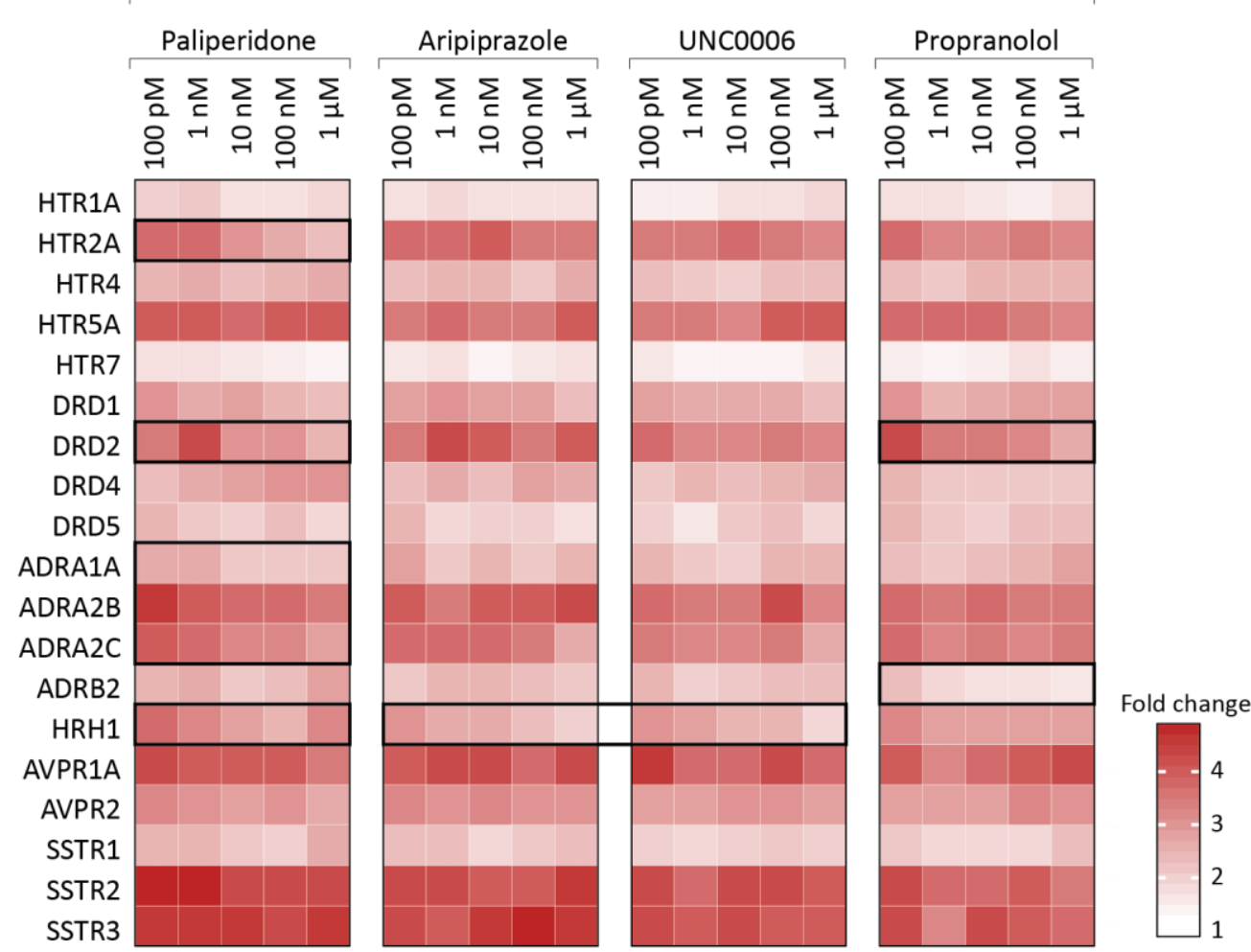

Figure 26: Profiles of drug effects on GPCR activation and inhibition.

Heatmap of drug effects on receptor activation measured in the multiplexed GPCR EXT assay. Plotted are fold changes of receptor activation in reference to unstimulated condition of 19 different GPCRs in U2OS cells. (A) Cells were treated with paliperidone, aripiprazole, UNC0006 and propranolol at the indicated concentrations. (B) Cells were treated with drugs as indicated together with agonist-mix containing the agonist serotonin, dopamine, epinephrine, histamine, vasopressin and somatostatin each at $1 \mu \mathrm{M}$. Agonist-mix was added $1 \mathrm{~h}$ after drugs. Observed dose-dependent receptor activations $(A)$ and inhibitions (B) are highlighted by black squares. 
A

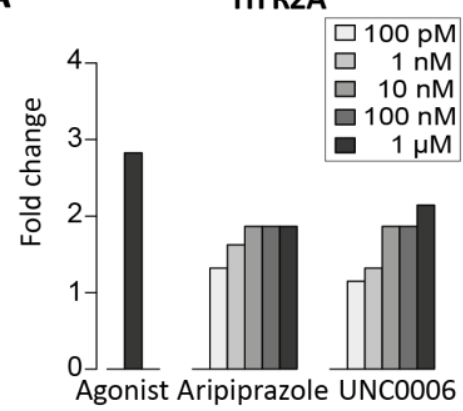

B

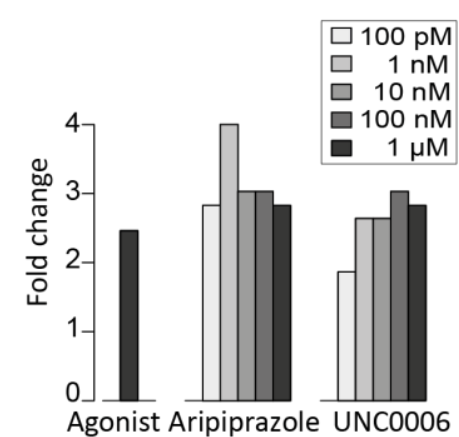

D
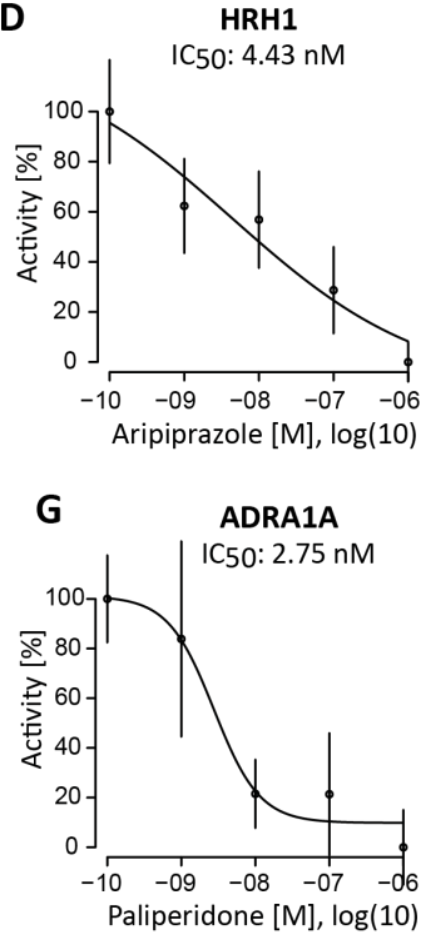

J

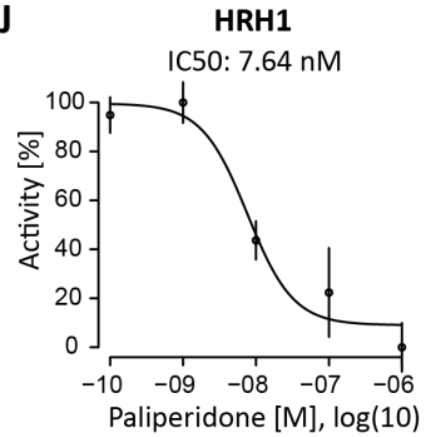

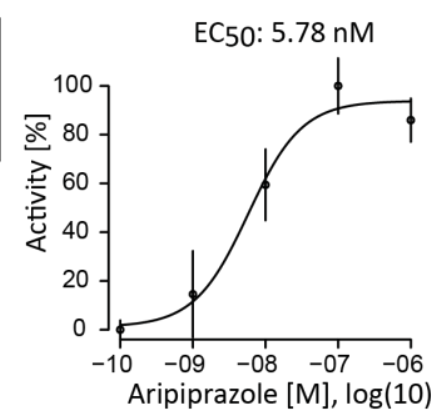

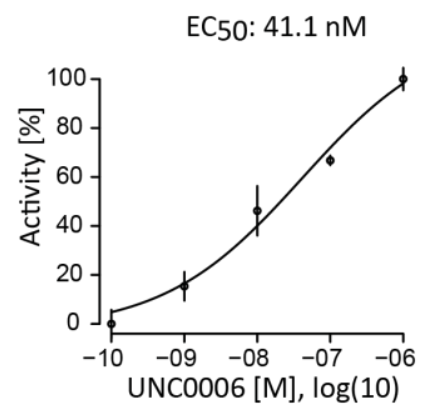

C

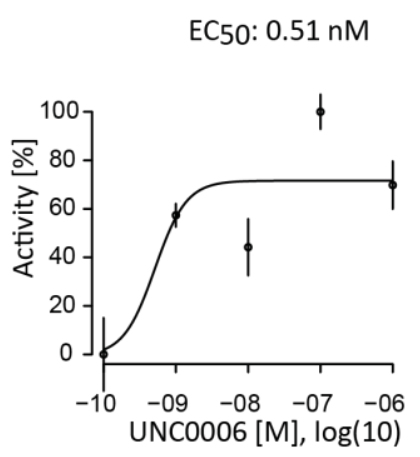

E
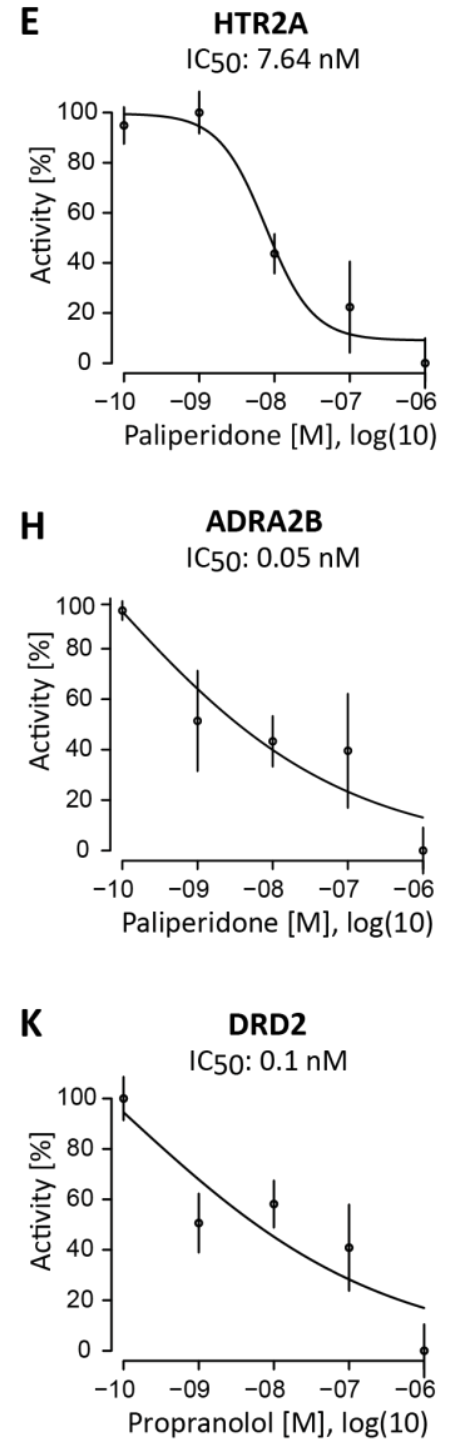

HRH1
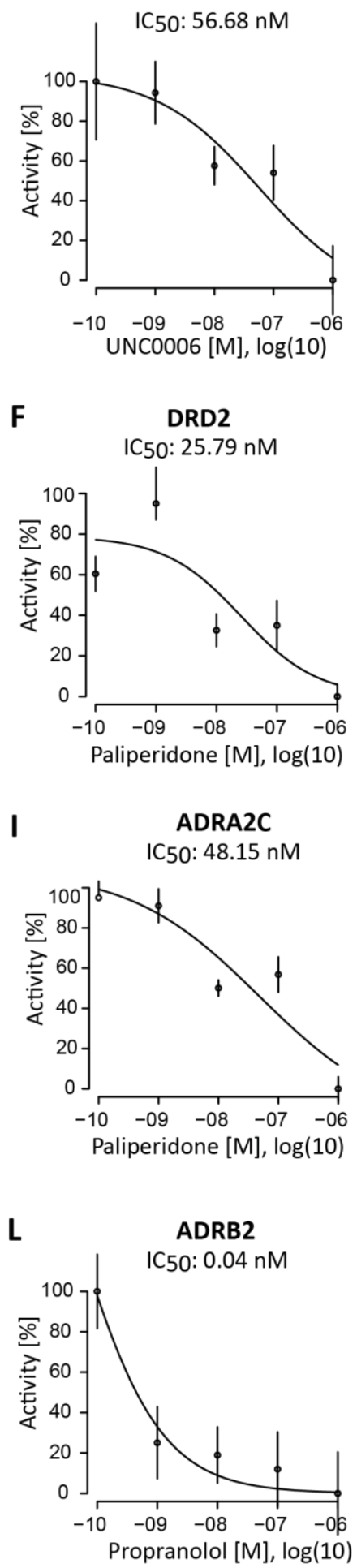
Figure 27: Analysis of drug effects on receptor activation in multiplexed GPCR EXT assay.

Bargraphs and dose-response curves of drug induced GPCR activation and inhibition in U2OS cells. Cells were treated with the particular drug alone (A and $B$ ) or with drug in the presence of an agonist-mix consisting of $1 \mu \mathrm{M}$ serotonin, dopamine, epinephrine, histamine, vasopressin and somatostatin (C-I). Data are given either as fold changes or percentage of activity of receptor activation of the mean of 2 replicates and 3 EXTs each; error bars represent standard error of the mean.

Next, to validate the effects caused by aripiprazole, paliperidone and propranolol and detected in the EXT-based approach, single luciferase-based dose response assays were performed. For aripiprazole, a dose-dependent activation of HTR2A and DRD2 with $\mathrm{EC}_{50}$ values of $125 \mathrm{nM}$ and $90 \mathrm{pM}$ as well as inhibition of $\mathrm{HRH} 1$ with an $\mathrm{IC}_{50}$ of 180 pM could be shown (Figure 28A-D). The dopamine receptor DRD2 showed a high affinity to aripiprazole reflected by the low $\mathrm{EC}_{50}$ of $90 \mathrm{pM}$, which may explain why no dose-dependent but rather a constant activation was detected in the EXT approach. The concentration range used in the EXT approach was outside the linear range of the dose response for the receptor ligand interaction. For paliperidone, all antagonistic effects observed in the EXT approach were also monitored in luciferase assays. Receptor inhibition was shown for HTR2A with an IC 50 of $4 \mathrm{nM}$, DRD2 with an IC 50 of $188 \mathrm{nM}, \mathrm{HRH} 1$ with an $\mathrm{IC}_{50}$ of $90 \mathrm{pM}$, as well as for the adrenergic receptors with $\mathrm{IC}_{50}$ values of $773 \mathrm{nM}$ for ADRA2B, and $177 \mathrm{nM}$ for ADRA2C (Figure 28D-H). Against the EXT-based data an inhibition of the dopamine receptor DRD2 by propranolol could not be validated in luciferase assays. The dose-dependent inhibition of the $\beta$-adrenergic receptor ADRB2 by propranolol resulted in an $\mathrm{IC}_{50}$ of $4.98 \mathrm{nM}$. 
A

HTR2A

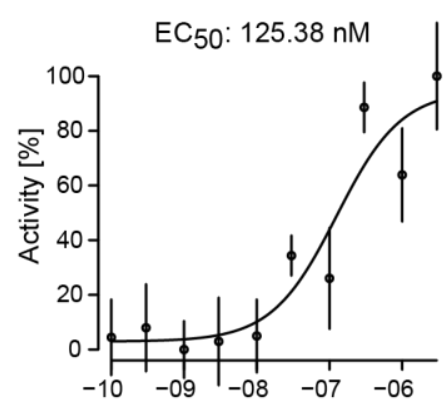

Aripiprazole [M], $\log (10)$

D

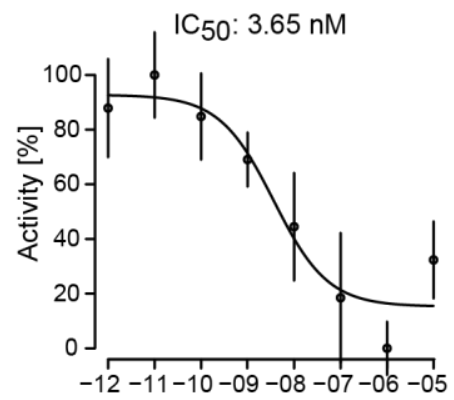

Paliperidone [M], $\log (10)$ $\& 1 \mu \mathrm{M}$ Serotonin

G

ADRA2B

$\mathrm{IC}_{50}: 773.14 \mathrm{nM}$

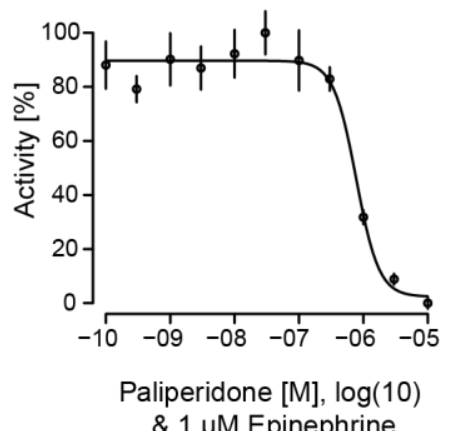

B

DRD2

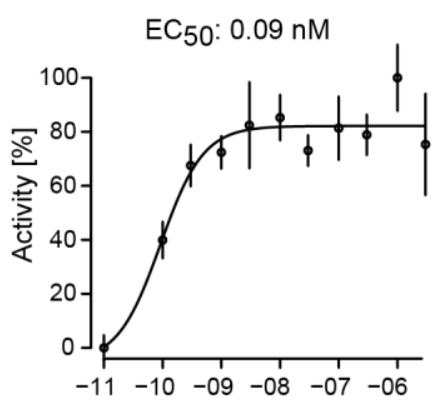

Aripiprazole [M], log(10)

E

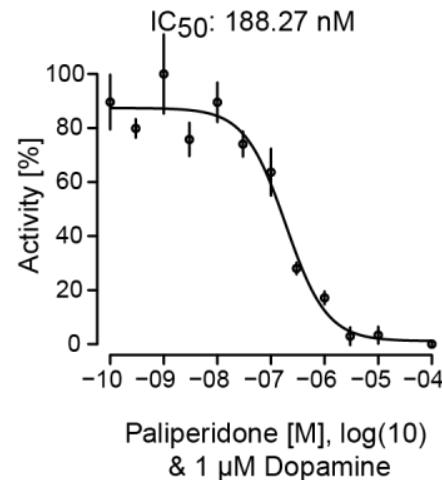

H

ADRA2C

IC $50: 177.92 \mathrm{nM}$

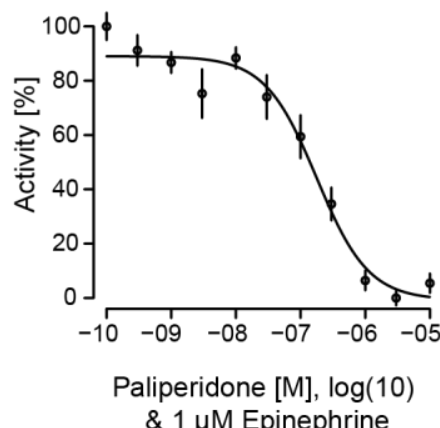

C

HRH1

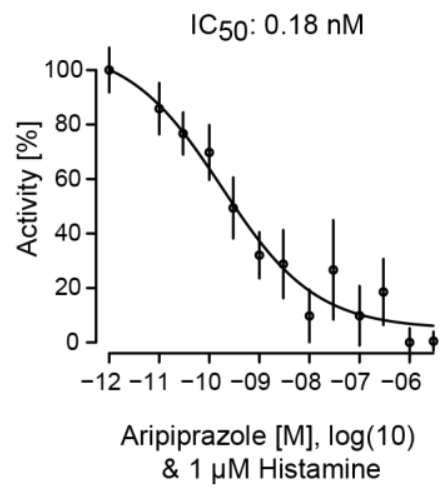

F

HRH1

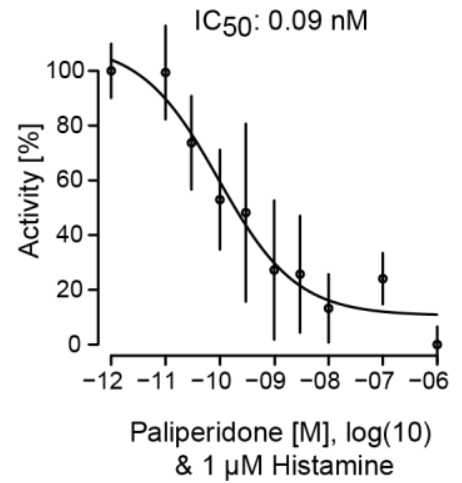

I

ADRB2

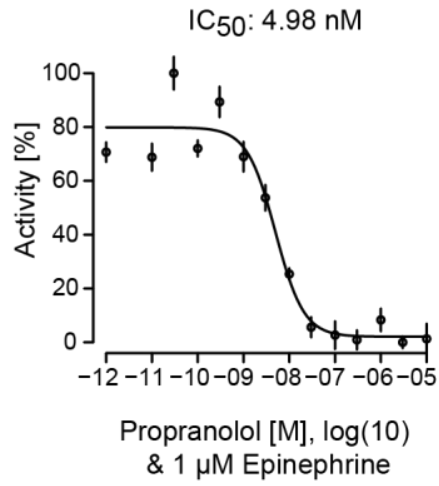

Figure 28: Luciferase-based validation of drug effects from GPCR EXT assay.

Dose-dependent GPCR activation and inhibition measured in single Luciferase assays in U2OS cells. Cells were treated for $6 \mathrm{~h}$ with the indicated drug (GPCR activation, $A$ and $B$ ) or with drug and corresponding agonist (GPCR inhibition, $C-1$ ). Dose-response curves and $\mathrm{EC}_{50}$ and $\mathrm{IC}_{50}$ values were calculated with $\mathrm{R}$. Luciferase data are given as means $\pm \operatorname{SEM}(n=6)$. 


\subsection{EXT-based measurement of GPCR downstream signalling}

\subsubsection{Principle of multiplexed GPCR signalling EXT assay}

GPCR-mediated signalling comprises the induction of multiple transduction pathways of $G$ protein-dependent and independent signalling cascades. Therefore, global downstream signalling responses initiated by GPCRs should be captured to obtain a comprehensive profiling map of GPCR activities. A method to monitor cellular events are cis-regulatory assays. Here, a library of sensor-EXT reporters containing different transcription factor binding sites (cis-elements) coupled to EXTs was applied to detect multiple GPCR mediated downstream events at the level of activated transcription factors (Figure 29A).

A

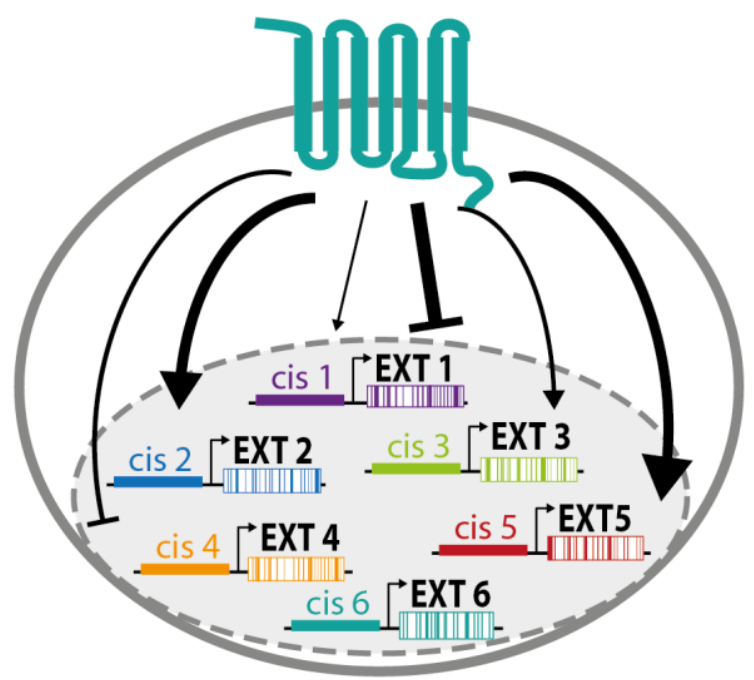

B

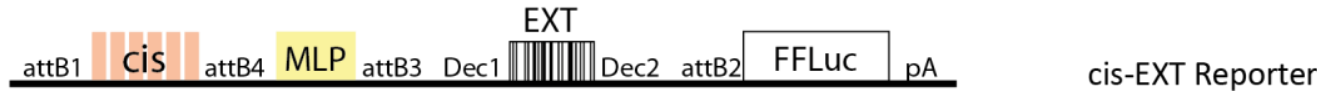

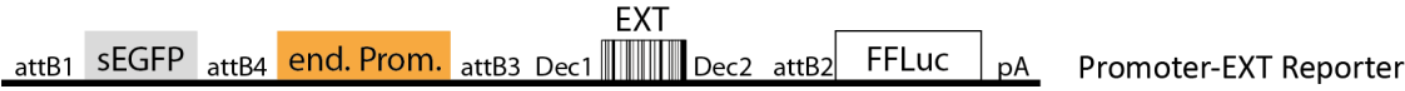

Figure 29: Schematic representation of EXT-based GPCR signalling assay.

(A) Graphical representation of EXT-based monitoring of GPCR downstream signalling using cisregulatory assays. Multiple sensor-EXT reporters encoding different transcription factor binding sites are simultaneously expressed to measure several signalling events at the level of activated transcription factors. (B) Modular structure of the two types of sensor reporter constructs for GPCR signalling assays carrying an EXT in front of the firefly luciferase gene (FFLuc). Cis-EXT constructs are under the control of a clustered cis-element followed by a minimal adenovirus major late promoter (MLP) whereas promoter-EXT constructs are under the control of an endogenous promoter. SEGFP stands for first $200 \mathrm{bp}$ of enhanced green fluorescence protein and is used as "dummy" for three fragment multisite Gateway recombination. attB1, attB2, attB3 and attB4 are recombination sites. 
Two types of EXT reporters were used, cis-EXT and promoter-EXT reporters. Cis-EXT constructs encode clustered single cis-elements and a minimal promoter, whereas promoter-EXT constructs encode 1-1.5 kb long regions located $5^{\prime}$ of endogenous promoters covering numerous regulatory elements (Figure 29B). Each sensor (cis-element and promoter) was assigned to 2-3 unique EXTs to generate a library of 264 sensor-EXT reporters (Table 1), which were used in multiplexed GPCR signalling EXT assays. The library also includes reporter constructs that contain neither a cis-element nor a promoter and serve as calibrators during the normalisation process of sequencing data. A list of sensors is shown in Table 4 in the Methods section.

\subsubsection{Profiles of GPCR-mediated downstream signalling}

The EXT-based GPCR signalling approach was applied to measure cellular signalling events induced by GPCRs in response to their corresponding agonists in comparison with signalling profiles derived from $\mathrm{G}$ proteins. For this purpose, GPCRs were selected that couple to the different types of G proteins. All used GPCRs are listed in Table 7 together with the corresponding $\mathrm{G}$ proteins and applied agonists. To reduce potential interference of receptor fusion tags as used for split TEV assays with cellular signalling, constructs encoding GPCRs fused to a small V5-tag were used. To monitor G proteininduced signalling events, constructs encoding constitutively active variants of $G$ proteins $G_{s}, G_{i}, G_{q}$ and $G_{12}$ were used.

Table 7: Overview of GPCRs and corresponding compounds used in GPCR signalling assay. *) Information of G protein coupling is based on the IUPHAR database (Southan et al., 2015).

\begin{tabular}{lllcl} 
Receptor subgroup & GPCR & Full fusion protein & G protein * & Agonist \\
\hline Serotonin receptors & HTR2A & HOOK-HTR2A-V5 & Gq & Serotonin (5-HT) \\
\cline { 2 - 5 } & HTR4 & HTR4-V5 & Gs & Serotonin (5-HT) \\
\hline Dopamine receptor & DRD1 & DRD1-V5 & Gs & Dopamine (DA) \\
\hline Adrenergic receptors & ADRA2C & ADRA2C-V5 & $\mathrm{Gi}$ & Epinephrine (Epi) \\
\cline { 2 - 5 } & ADRB2 & ADRB2-V5 & $\mathrm{Gs}$ & Epinephrine (Epi) \\
\hline Histamine receptor & HRH1 & HRH1-V5 & $\mathrm{Gq}$ & Histamine (His) \\
\hline Somatostatin receptor & SSTR1 & SSTR1-V5 & $\mathrm{Gi}$ & Somatostatin (SST)
\end{tabular}


Signalling events were measured in PC12 tetOFF cells using the sensor EXT library under the conditions of constitutively active $\mathrm{G}$ proteins, unstimulated and stimulated GPCRs, and ligand administration in absence of receptors (Figure 30A). For each condition, separate on-plate transfections of the sensor-EXT library alone, with GPCRs or $\mathrm{G}$ proteins were performed. Cells expressing sensor library with and without GPCRs were treated according to the experimental workflow of agonist assays and stimulated for $6 \mathrm{~h}$ with $1 \mathrm{\mu M}$ of the corresponding agonist. Cell samples expressing active $\mathrm{G}$ proteins were harvested $24 \mathrm{~h}$ after transfection. The RNA from all samples was isolated and processed as described for previous experiments (Figure 20B). The raw sequencing data was processed according to previous experiments by normalisation, consolidation and standardisation. To balance the clarity of results and the stringency of the experiment not all sensors were included in the final signal profiles based on analyses of the variances of sensor performance (Figure 30B).

A

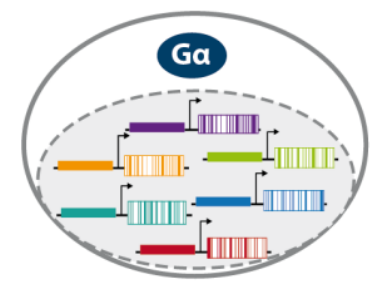

G proteins

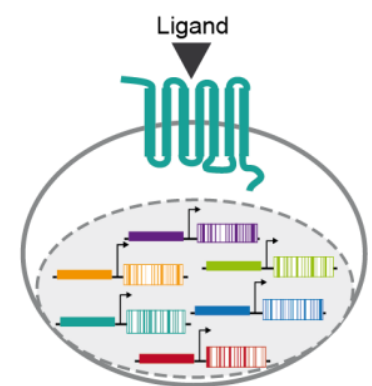

GPCRs

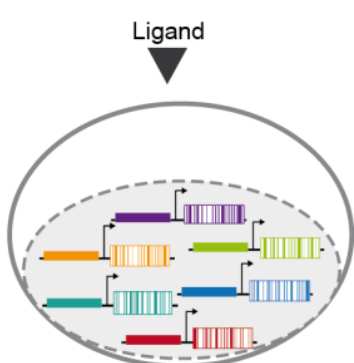

Ligands

B

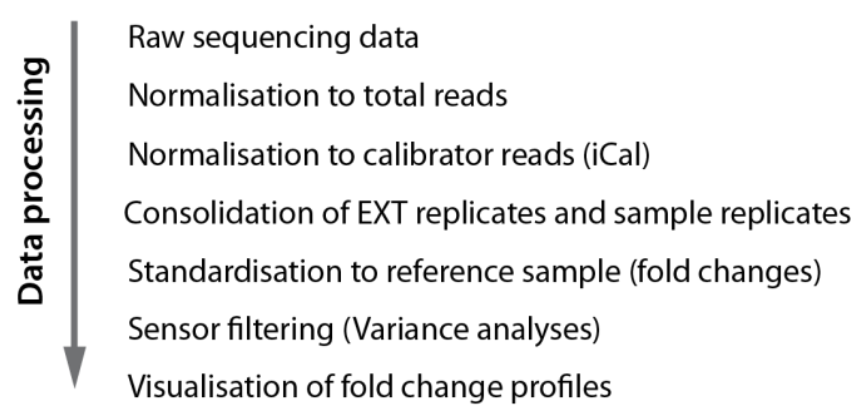

Figure 30: GPCR signalling assay for monitoring GPCR and G protein-induced signalling.

(A) Experimental design of the three different condition parts within the GPCR signalling assay. To monitor $G$ protein-induced signalling constitutive active alpha subunits of $G$ proteins were expressed (left). To monitor stimulation-induced GPCR signalling GPCRs were expressed as V5 fusion constructs and stimulated with corresponding ligands (middle). As control for endogenous signalling ligands were applied in absence of GPCRs (right). (B) Workflow of data processing after next generation sequencing of GPCR signalling EXT assays. 
Taken together, signalling profiles with a high similarity as well as with clear differences were obtained affecting a broad range of pathway sensors (Figure 32). Baseline activities of endogenous GPCR signalling were low in PC12 cells, as seen in control experimental conditions of ligand exposure in absence of exogenous GPCRs.

Using this assay, correlations between signalling profiles were determined. Here, high similarities between particular GPCR induced profiles were observed (Figure 33) as well as relationships of individual GPCR induced profiles to particular G protein mediated profiles (Figure 34). The observations are thereby in accordance with the described preferential G protein coupling (Table 7). HTR2A and HRH1 preferentially couple to $G_{q}$. Both receptors showed strong, high similar signal profiles and form a cluster together with the $\mathrm{G}_{\mathrm{q}}$ profile separated from all other samples (Figure 33 and Figure 34). The profiles of DRD1 and ADRB2 were closely related to each other the first level of clustering and at the second level to HTR4A. All three GPCRs preferentially couple to $G_{s}$. However, the $G_{s}$-induced profile was distantly clustered. The activation of the $G_{i}$-coupled SSTR2 exhibits a similar signalling pattern as the $G$ protein $G_{i}$ (Figure 34). In contrast ADRA2C, which is also described to couple to $G_{i}$ showed a signal profile that is different from all other profiles, with the majority of pathway sensors being downregulated.

The highest regulatory effects for all GPCRs and G proteins were observed for the CRE, UPRE and SARE cis-elements and the 118 and NR4A1 promoters. Here, the reporters CRE and UPRE and II8 and NR4A1 display a highly comparable response. CRE is one of the most prominent cis-elements commonly used in reporter gene assays for monitoring GPCR activities. Based on the literature for general G protein signalling and preferential coupling to GPCRs, the obtained results for the CRE-reporter can be compared with other predictions. The EXT-based results for $\mathrm{G}$ protein-induced CRE reporter activities are compared with the activation patterns derived from an independent luciferase assay (Figure 31). By regulating the intracellular cAMP level, $\mathrm{G}_{\mathrm{s}}$ protein activity caused an increase in reporter activity, whereas the signals for $G_{i}$ were downregulated (Figure 31). Elevated signals were also detected for the $\mathrm{G}_{\mathrm{s}}$-coupled GPCRs HTR4, DRD1, and ADRB2, as well as a downregulation for the $G_{i}$-associated SSTR2 and ADRA2C (Figure 32). CRE cis-element activity is driven by the transcription factor CREB, which in turn is activated by the CAMP pathway. In addition, CREB is 
described to be activated by cellular calcium (Grewal et al., 2000; Johnson et al., 1997). As expected, the $G_{q}$ protein as well as the $G_{q}$ coupled GPCRs HTR2A and HRH1 showed increased activation signals (Figure 32). The SARE reporter showed high activation signals in the $G_{q}, G_{12}, H T R 2 A$ and $H R H 1$ samples. The $G_{12} G$ protein is not described to be coupled to one of the used GPCRs. The highest activation signals induced by $\mathrm{G}_{12}$ could be observed for SARE, SRF and JUN promoters (Figure 32). The obtained signalling profiles of the $\mathrm{G}$ proteins were to some extent similar to the corresponding GPCRs; however, differences were observed. For example, Myc-E-box responses were, increased by $\mathrm{G}_{\mathrm{q}}$ signalling, but decreased by HTR2A and HRH1 actions.

A

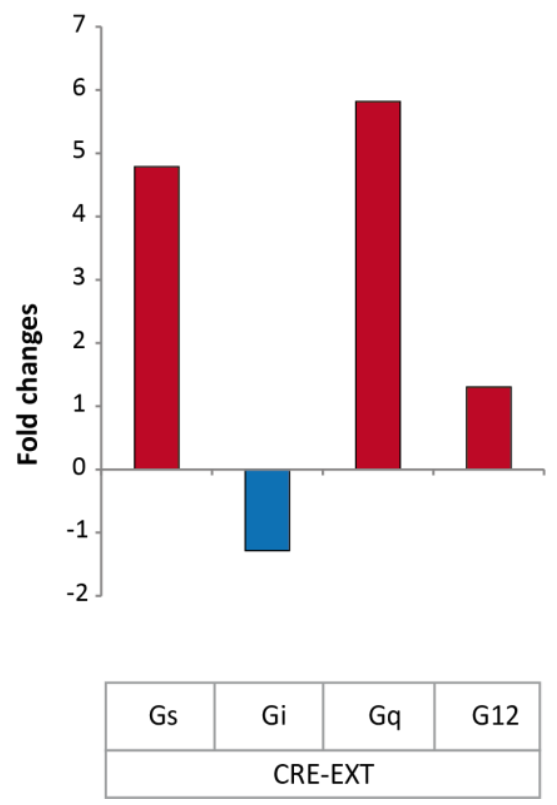

B

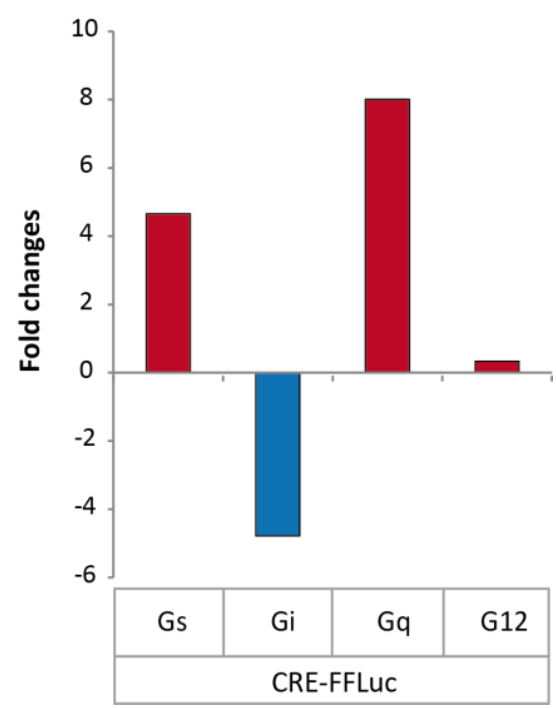

Figure 31: Activity signals of $G$ proteins on CRE reporter by EXT vs. luciferase measurement.

Comparison of CRE-reporter activity by active $G$ proteins measured in the EXT-based signalling assay (A) and in an independent luciferase assay (B). Plotted are the fold changes of reporter activity by constitutively expressed $\mathrm{G}$ proteins. Figure (A) shows the extracted data from the multiplexed assay of the pooled EXT signals of two CRE-reporters encoding unique EXTs. Figure (B) shows the luciferase signals of one CRE-reporter construct. In both assays the $G$ protein and reporter constructs were transfected into PC12 cells and expressed for $24 \mathrm{~h}$. EXT and luciferase measurements display similar activity patterns comparing $G$ proteins. 


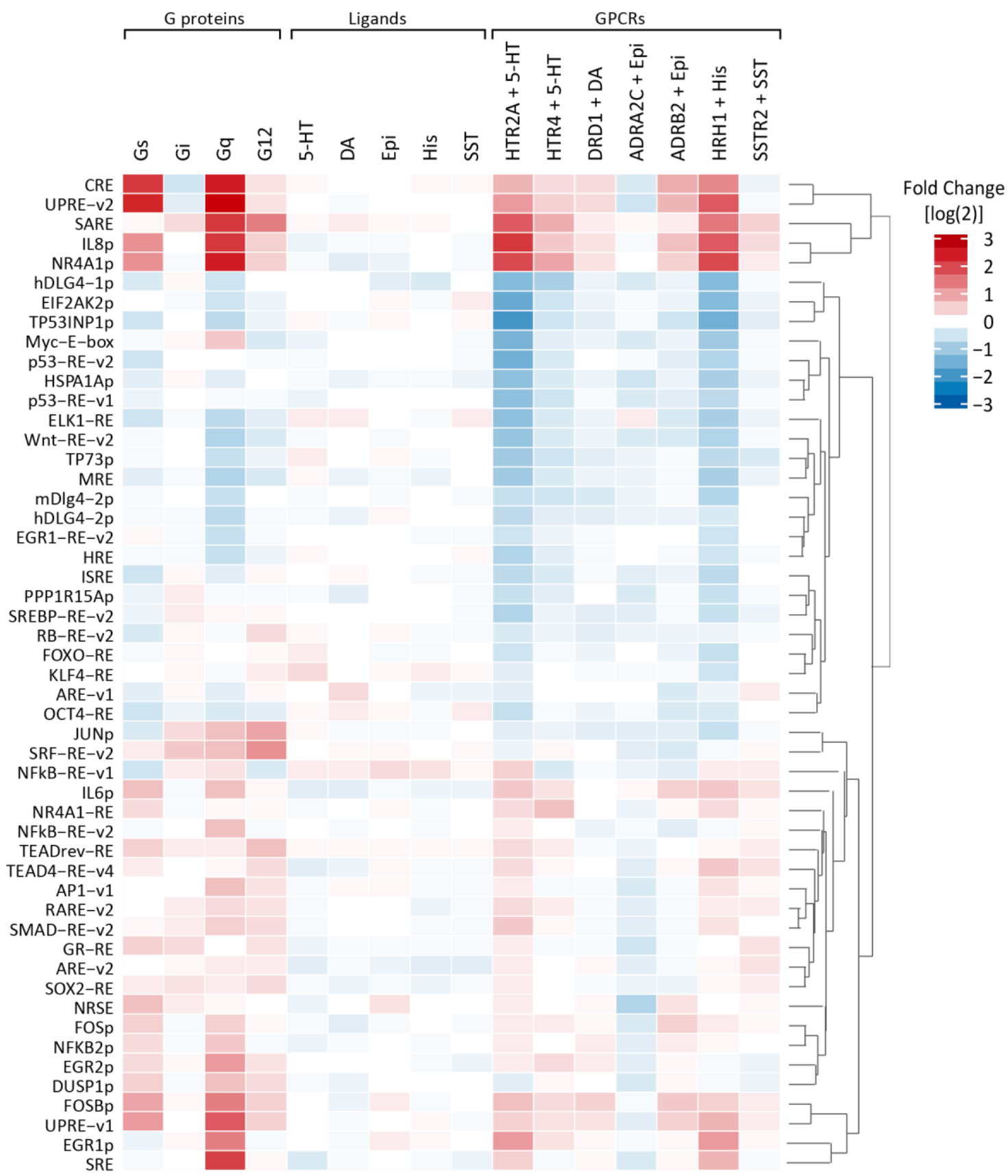

Figure 32: Signal profiles of $G$ proteins and GPCRs using an EXT sensor library.

PC12 tetOFF cells were transfected on-plate with assay components comprising constitutive active $G$ proteins and different GPCRs. Receptors were stimulated with $1 \mu \mathrm{M}$ of corrsponding agonists for $6 \mathrm{~h}$. As control cells were exposed to ligands in absence of receptors. For each assay condition the log foldchanges of the different EXT reporters in reference to basal reporter activities and unstimulated GPCRs respectively are plotted. 


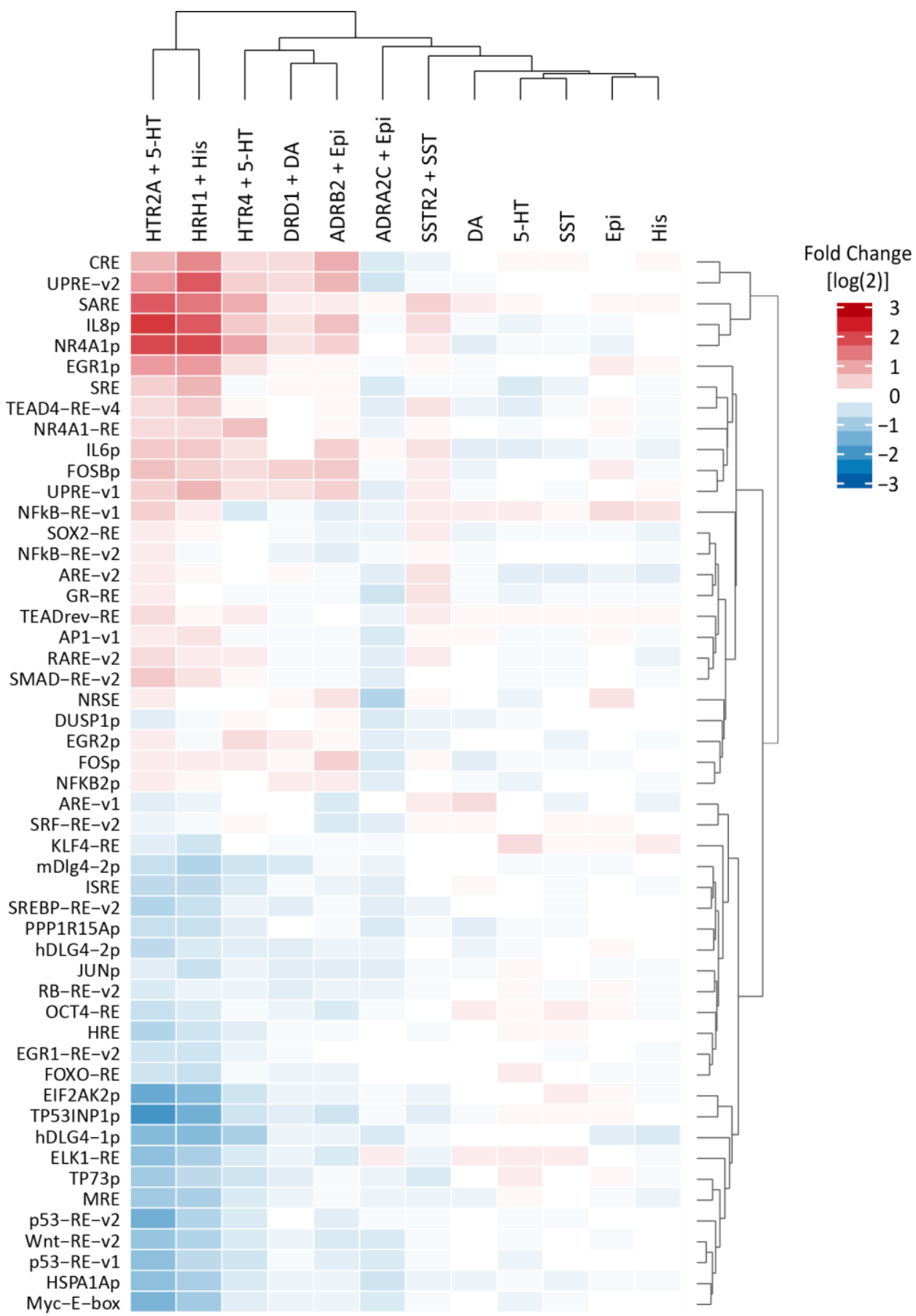

Figure 33: Clustering of GPCR induced signalling profiles and control conditions.

Selection of data from the multiplexed GPCR signalling assay for the comparison of GPCR induced downstream profiles by receptor stimulation and the exposure in absence of exogenous receptors. The hierarchical clustering revealed close relationships between groups of GPCRs. 


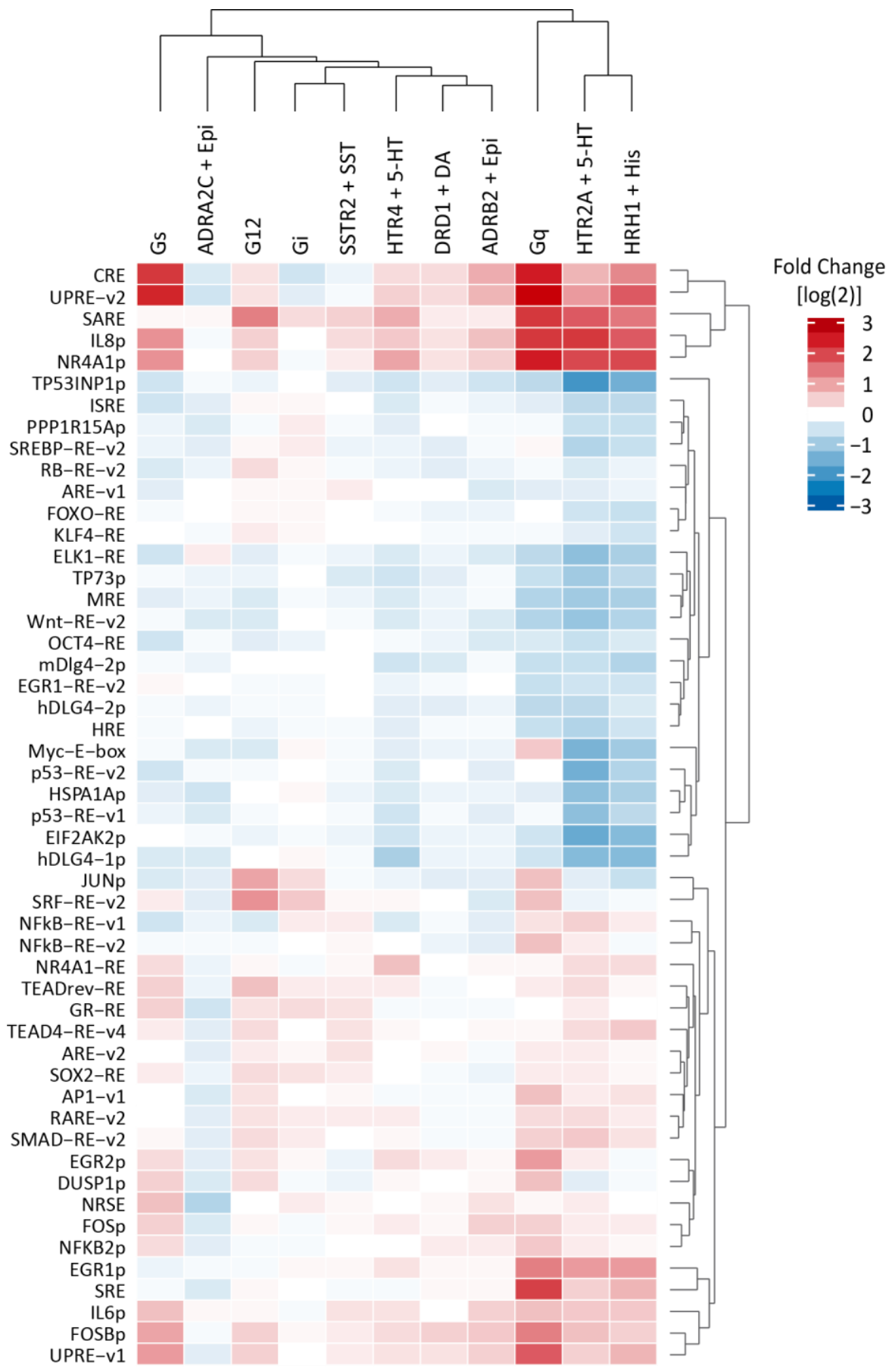

Figure 34: Clustering of G protein and GPCR induced signalling profiles.

Comparison of selected signal profiles of active $G$ proteins and ligand-activated GPCRs from the multiplexed GPCR signalling assay. The cluster analysis showed relations between $\mathrm{G}$ proteins and preferentially associated GPCRs. 


\section{Discussion}

\subsection{Multiplexing of GPCR activation assays}

Several methods are available to assess ligand actions on GPCRs. The most classical approach to identify ligand receptor interactions are cell-free binding assays. These assays have the intrinsic disadvantage that compounds are frequently radioactively labelled and that binding affinity data does not provide any information on the functional properties of ligands. Thus, it is neither clear whether ligands act as agonist or antagonist, nor which efficacies are exerted under physiological conditions (Zhang and Xie, 2012). These shortcomings are overcome in cell-based functional assays. These approaches address ligand-dependent receptor activation and signalling within a cellular context. Most of these approaches rely on receptor-mediated activation of $G$ proteins, either by direct measuring of second messenger levels (e.g. CAMP and calcium) (Emkey and Rankl, 2009; Gilissen et al.; Williams, 2004) or by reporter gene assays that respond to second messenger activities (Cheng et al., 2010). Monitoring multiple GPCR-ligand interactions within one experimental setup was, however, not feasible as of today different G proteins couple to various types of GPCRs.

A sensitive and robust method to monitor receptor activation of GPCRs uses the dynamic recruitment of $\beta$-arrestin, which is a measurement independent of $G$ protein activity. Recently, a large collection of cell-based assays based on $\beta$-arrestin recruitment was introduced to interrogate the druggable GPCRome in a parallel manner (Kroeze et al., 2015). The assay uses a modified Tango assay (Barnea et al., 2008) for screening compound libraries against a multiplicity of orphan and nonorphan GPCRs. However, the approach is based on the parallel measurement of single one compound - one target assays. Therefore, adding additional GPCRs and/or compounds to screening conditions will lead to extensive consumption of materials at increasing costs.

In this thesis, a multiplexed GPCR activity assay was developed to profile many GPCR activities simultaneously. Within such an assay, multiple GPCRs can be screened at various conditions, which, for examples, includes the addition of several ligands. The multiplexed assay uses both EXT molecular barcode reporter and split TEV 
technologies to optimise data point acquisition within one assay. GPCR activity profiles induced by both natural agonists and therapeutic drugs were determined in proof-ofprinciple experiments.

\subsubsection{Selection of GPCRs}

One application of the multiplexed activation assay should be the assessment of polypharmacological activation profiles of antipsychotic drugs. Therefore, GPCRs were selected that are associated with the action of antipsychotic drugs. Most of the clinically used antipsychotics show high binding affinities to the subgroups of serotonin, dopamine, adrenergic, and histamine receptors (Roth et al., 2004). To obtain a manageable number of GPCRs, five representative GPCRs from each group and the histamine receptor HRH1 were selected. All GPCRs of the "assay group" are monoamine-binding GPCRs- For a control group, GPCRs were selected that bind to unrelated ligands, such as the peptide-binding vasopressin and somatostatin receptors. Importantly, all GPCRs chosen showed a robust performance in split TEV assays.

\subsubsection{Time limitations of GPCR split TEV assays}

A critical aspect when setting-up an assay monitoring multiple receptor activities simultaneously is the determination of the duration of the stimulation phase and thereby the time point of measurement. Therefore, the kinetic behaviour of selected GPCRs was analysed in split TEV assays to get an estimation for the best stimulation time. For all GPCRs. similar kinetics of increasing activity signals, reaching a maximum, followed by decreasing signals were observed, thus displaying the typical kinetic of receptor activation and desensitisation in this assay system. Notably, activation profiles indicated that GPCR/ $\beta$-arrestin recruitment- assays reach their peak after $4-8 \mathrm{~h}$. All GPCRs tested displayed comparable activation kinetics, with the exception of the dopamine receptor DRD1 showing an advanced kinetic. Based on these results, the stimulation phase for all further GPCR split TEV assays was set to $6 \mathrm{~h}$, covering all 
receptor activation phases including early and delayed ones. However, it should be noted that shifted peaks of activation have to be considered when assaying multiple receptors at the same time point as measurable profiles may lie beyond the measurement point.

\subsubsection{Split TEV assays as tool for monitoring GPCR activations}

For efficient combination of the EXTassay and split TEV technique for multiplexed assays, first the applicability of split TEV assays under the developed experimental conditions/workflow was tested for all GPCRs in single luciferase assays. For each GPCR, a significant stimulation-dependent activation was successfully detected in at least one cell line.

Further, it was shown that the GPCR split TEV assay is functional to monitor not only the agonist-induced activation bur also the antagonist-induced inhibition of receptor activity. In addition, the split TEV assay proved to be sensitive to the applied stimulation concentration resulting in dose-response analyses. The obtained $\mathrm{EC}_{50}$ and $I_{50}$ values are comparable with reported results for binding affinities of these receptor ligand combinations (Southan et al., 2015).

Considering the receptor assay performance, differences between cell lines were observed. For example, all serotonin receptors showed only tendencies but no distinct receptor activation after stimulation in $\mathrm{CHO}$ cells, but performed to some extent in PC12 cells, and very well in U2OS cells. In contrast, dopamine receptors performed well in U2OS and CHO cells, but not in PC12 cells, a finding also previously reported (Djannatian et al., 2011). A possible explanation is that PC12 cells are described to synthesize dopamine (Greene and Tischler, 1976) and that this endogenous dopamine is sufficient to activate the receptors. For instance, dopamine receptors would get permanently activated and the exogenous applied dopamine cannot further increase the receptor activation. Another explanation may be that different expression profiles of the exogenous assay components exist, especially for GPCR assay constructs. The degree of expression and cell surface localisation may differ among cell lines. U2OS cells proved to be the most suitable cell line for multiplexed GPCR activation assays, as nearly all selected GCPRs showed significant activations. The observed differences in 
the assay performance confirm the importance of the cell type used for functional GPCR assays. The cellular background regarding the synthesis of endogenous ligands but also localisation and abundance of proteins within the GPCR regulatory cycle, such as G proteins and GRKs may influence assay performance. Therefore, when establishing GPCR split TEV assays to be used in multiplexed assays, it is key to consider cell type-dependent differences related to sensitivity, robustness, and kinetics.

\subsubsection{GPCR activity profiles monitored in multiplexed split TEV assays}

Split TEV assays have the advantage of providing a highly flexible choice of readout. In addition, EXT barcode reporters are a flexible and scalable readout system for an integrated analysis of multiple cellular events making EXTs an excellent as readout tool for multiplexed GPCR split TEV assays to assess a matrix of GPCR compound interactions. Firstly, experiments were done to profile actions of natural agonists to the selection of different GPCR subgroups. The profiles obtained show activation patterns that are specific for each agonist. The monoamine neurotransmitter serotonin as well as the peptide hormones vasopressin and somatostatin are highly specific as they only activate their corresponding receptor group. In contrast, the catecholamines dopamine and epinephrine are able to activate both dopamine and adrenergic receptors, a finding most likely owing to the highly similar structure of both ligands. Dopamine, epinephrine and norepinephrine belong to the same synthesis pathway, while dopamine is the precursor of epinephrine, which is further converted to norepinephrine. The observation of cross-activation of adrenergic receptors by dopamine and vice-versa is supported by findings in the literature (Lanau et al., 1997; Rey et al., 2001; Zhang et al., 2004).

The general activation profiles showing the ligand specificity were consistent between the human U2OS and rat PC12 cell lines. However, in PC12 cells, some GPCRs, such as the dopamine receptors DRD2-5 and the serotonin receptors HTR1A and HTR7, showed no marked responses to any of the stimuli. This observation is comparable to the luciferase-based activation data, where for these GPCRs also no stimulation- 
dependent activation in PC12 cells could be detected, supporting the notion that cellular background has a fundamental influence on the assay performance.

\subsubsection{Profiling the action of drugs on receptor activation}

In drug discovery, it is important to assess the functional properties of a given drug, as a drug may acts as agonist, partial agonist, or antagonist. GPCR assays monitoring antagonistic effects implicate a co-treatment of antagonist and a GPCR corresponding agonist. In multiplexed GPCR activity assays, numerous GPCRs activated by different agonists are implemented within one mixed cell population. Therefore, the activation of individual GPCRs by a compound mix was applied that contained all agonists for the GPCRs tested. The application of the agonist mix revealed the same activation pattern as the applied single compounds. The overall activation strength of the individual receptors was comparable between mixed and single agonists. Therefore, antagonistic effects can be monitored in multiplexed assays using a co-treatment paradigm based on agonist mixtures that keep stimulation conditions and sample numbers down when compared to applying the associated agonists separately.

However, the functional property of a particular drug is not always known. For example, aripiprazole the drug is reported to act functionally selective as agonist and antagonist depending on both the receptor and cell type (Shapiro et al., 2003). For a proof-of-principle experiment to monitor drug effects, substances were chosen that have known receptor affinities and functional properties. To detect antagonistic but also agonistic drug effects, the multiplexed activation assay was performed under the two parameters of drug treatment alone and in the presence of agonists.

The atypical antipsychotic drug aripiprazole and its analogue UNC006 were monitored in the assay as DRD2 and HTR2A agonists and HRH1 antagonist, and these findings were validated in single luciferase assays. The activation of HTR2A is contrary to the literature where an antagonism for this receptor is usually described. Conversely, a weak partial agonistic effect was reported for aripiprazole in GF62 cells (Shapiro et al., 2003). It may be possible that aripiprazole acts functionally selective not only on DRD2 (Lawler et al., 1999) but also on HTR2A in a cell type- dependent manner. For aripiprazole, an additionally partial agonism on the serotonin receptor HTR1A is 
reported, which was not, however, detected in this multiplexed activation assay. An explanation can be that a partial agonism implies the activation of a receptor with only partial efficacy in contrast to a full agonist and that this partial efficacy was too low to be detectable in the split TEV assay, indicating a potential limited sensitivity of the assay. Taken together, it cannot be concluded if monitored agonistic effects are based on a partial or full agonist. For this purpose, the ligand has to be monitored in direct comparison to a full agonist (endogenous agonist) to compare the efficacies.

In contrast to aripiprazole, paliperidone is a more classical atypical antipsychotic drug that acts only as full antagonist. The monitored inhibitory effects on HTR2A, DRD2, the $\alpha$-adrenergic receptors and HRH1 are consistent with the literature for all GPCRs included in the assay (Mauri et al., 2014).

Propranolol is a beta blocker and specifically acts on $\beta$-adrenergic receptors. A strong effect on ADRB2 by propranolol could be assessed and validated by further luciferase measurements. Furthermore, the EXT assay showed an inhibitory effect on the dopamine receptor DRD2, which was invalidated by single luciferase assays.

$\mathrm{EC}_{50}$ and $\mathrm{IC}_{50}$ values were calculated using dose-response assays run with EXT and luciferase readouts. Notably, EXT-derived values partially differ from luciferase-based measurements for the same GPCR ligand combination. Here, it has to be mentioned that the $\mathrm{EC}_{50} / \mathrm{IC}_{50}$ values obtained from the multiplexed EXT assay are at most semiquantitative due to the low number of different concentrations and the wide range between the individual dilutions. In contrast, the luciferase-based dose-response assays comprise a higher range of dilutions with higher resolution. Another aspect that might contribute to the analysis of EXT-based dose response curves is the method of read calibration during data processing. An improved method to calibrate sequencing data might enhance the robustness between the different samples/conditions. Taken together, the obtained $\mathrm{EC}_{50} / \mathrm{IC}_{50}$ values are approximate values within a picomolar to nanomolar range comparable with published affinity (Ki) values (Southan et al., 2015). In summary, GPCR activity assays were applied to monitor dose-dependent activity profiles of drugs and known effects caused by these drugs. In this setup, both agonistic as well as antagonistic effects by the same drug were assessed in parallel. 


\subsection{Profiling of GPCR downstream signalling}

The multiplexed signalling assay was designed to address the complex cellular signalling mediated by active GPCRs. In a proof-of-principal experiment, signal profiles were obtained in response to GPCR activation and by expression of constitutively active G protein variants. Ligand-activated GPCRs revealed signal patterns that are highly similar to those caused by associated $G$ protein variants only, supporting the preferential G protein coupling of GPCRs, which is consistent with published data. The differences observed between $\mathrm{G}$ protein and GPCR-derived profiles indicate the participation of other effector proteins, such as $\beta$-arrestin involved in GPCR-mediated signalling other than the classical G proteins.

The most prominent activity for GPCR signalling was observed for CRE cis-reporterdependent signals, which is in concordance with the known regulation of second messengers by the different types of $G$ proteins. CRE was activated by $G_{s}$ and coupled GPCRs as well as by $G_{q}$ corresponding receptors, while $G_{i}$ coupled GPCRs showed downregulated signals. The observations classify CRE not only as classical sensor for GPCR induced CAMP signalling but also for calcium signalling (Grewal et al., 2000). Further, high signals were observed for the UPRE cis-element, and the IL8 and NR4A1 promoters. The unfolded protein response element (UPRE) is involved in the response to stress in the endoplasmic reticulum (ER). IL8 is part of the cytokine signalling response and is activated by the transcription factors AP-1 and NFkB (Hoffmann et al., 2002). NR4A1 is an immediate-early gene, or stress response gene, that is activated by a variety of stimuli, such as serum or neurotransmitters (Hazel et al., 1988; Maxwell and Muscat, 2006). Notably, both promoters contain a CREB binding site (Bezzerri et al., 2011; Inaoka et al., 2008) suggesting that activation patterns similar to CRE reporter-dependent signals may be induced by $\mathrm{G}$ proteins. To analyse the regulation of particular sensors in more detail, further experiments for the validation of the activation mechanisms of the sensors are required. For example, overexpression of pathway specific kinases could be used to classify signalling activities and performances of selected sensors, and provide guidelines to establish molecular phenotypes of drugs and compounds of interest. 


\subsection{Concepts and future aspects of GPCR profiling assays}

The GPCR activity assay enables the rapid and easy detection of receptor activities for multiple GPCRs in response to several ligands within one experiment compared to conventional single assays wherein each receptor-ligand combination has to be measured separately. The modular structure and robust performance using transient transfections make the assay highly flexible and scalable in terms of combinations of receptors and ligands. Applying this flexibility, stimulation-dependent interactions of receptors with other adapter proteins could be included. For example, GPCR-adapter interaction assays other than $\beta$-arrestin2 might be useful for further screening questions addressed. The application of the assay by transient transfections is time and cost effective as well. However, stable cell lines expressing the adapter protein and/or reporter constructs might enhance the robustness and sensitivity of the assay, especially for weak receptor activations caused by partial agonists.

Receptor activation profiles are generated that could be useful in drug discovery to find novel and unexpected targets and to understand drug actions related to therapeutic and adverse effects (Allen and Roth, 2011). An in vitro pharmacological profiling of compound actions on a panel of selected targets during early stages of drug discovery could contribute to reduced attrition rates at late and more expensive clinical stages of the process due to early observed off-target effects (Bowes et al., 2012; Papoian et al., 2015).

The GPCR signalling assay enables a comprehensive detection of multiple ligand mediated GPCR signalling events within one analysis. Signal profiles of GPCRs, activated by natural agonists could be successfully monitored. As a next step, profiling of drug-mediated GPCR signalling might be applied to get insight into the action of drugs on cellular signalling. Results of drug-induced receptor activations obtained from the GPCR activity assay could be further analysed regarding the cellular downstream effects. Furthermore, the assay could be applied in the analysis of ligands that act functionally selective. The assay might be implemented in the research of functional selective drugs with enhanced therapeutic and reduced adverse effects (Kenakin and Christopoulos, 2013; Whalen et al., 2011). Specific G protein-dependent and 
independent signalling pathways could be monitored, for example, by silencing the expression of $\mathrm{G}$ proteins or $\beta$-arrestins using shRNAs or CRISPR/Cas9 technologies.

For monitoring dynamic changes in pathway signalling and provide dynamic profiles, compound actions could be determined at different time points.

The assay is applicable by transient transfections, making it flexible in handling. However, transient transfections are not suitable for all cell types. For monitoring drug-induced GPCR signalling, more specific cell types should be used for profiling effects of antipsychotic drugs. These cell types, are however, often hard to transfect, such as primary neuronal cells. The production of viruses from the sensor library will be a suitable method to circumvent these problems and will make it feasible to target these cell types (i.e. Adeno-associated viruses for primary neurons). Furthermore, an adaptation to microtiter plate formats (96- or 384-well) would make the approach suitable for high throughput analyses.

In summary, the profiling system presented comprising the two concepts of multiplexed assays, constitute a comprehensive approach for an extensive analysis of GPCR activities and downstream signalling. The approach offers a screening application in drug discovery and development for profiling compound actions in a comprehensive and time and cost effective way. More information about drugs developed might uncover off-target and off-pathway effects, lower expensive attrition rates or provide a better understanding of complex on-target and pathway effects towards complex diseases. 


\section{Abbreviations}

\begin{tabular}{|c|c|}
\hline $5-\mathrm{HT}$ & 5-hydroxytryptamine (serotonin) \\
\hline$A C$ & Adenylate cyclase \\
\hline Amp & Ampicillin \\
\hline ADP & Adenosine diphosphate \\
\hline ATCC & American Type Culture Collection \\
\hline ATP & Adenosine triphosphate \\
\hline AVP & Arginine vasopressin \\
\hline BSA & Bovine serum albumin \\
\hline CAMP & $3^{\prime}, 5^{\prime}$-cyclic adenosine monophosphate \\
\hline CNS & Central nervous system \\
\hline $\mathrm{Cm}$ & Chloramphenicol \\
\hline CMV & Cytomegalus virus \\
\hline CRE & cAMP response element \\
\hline DA & Dopamine \\
\hline DAG & Diacylglycerin \\
\hline DMEM & Dulbeccos modified eagle medium \\
\hline DMSO & Dimethylsulfoxide \\
\hline DNA & Deoxyribonucleic acid \\
\hline DNase & Deoxyribonuclease \\
\hline dNTP & Deoxyribonucleotide \\
\hline DTT & 1,4-dithiothreitol \\
\hline $\mathrm{EC}_{50}$ & Half maximal effective concentration \\
\hline E.coli & Escherichia coli \\
\hline EDTA & Ethylenediamine tetraacetic acid \\
\hline EGFP & Enhanced green fluorescent protein \\
\hline e.g. & For example \\
\hline Epi & Epinephrine \\
\hline ERK & extracellular signal-regulated kinase \\
\hline et al. & And others \\
\hline EtOH & Ethanol \\
\hline
\end{tabular}




\begin{tabular}{|c|c|}
\hline EXT & Expressed oligonucleotide tag \\
\hline f.c. & Final concentration \\
\hline FBS & Fetal bovine serum \\
\hline FDA & U.S. Food and Drug Administration \\
\hline Gal4 & Yeast DNA binding domain \\
\hline GDP & Guanosine diphosphate \\
\hline Genta & Gentamycin \\
\hline GFP & Green fluorescent protein \\
\hline GPCR & G-protein coupled receptor \\
\hline GRK & G protein-coupled receptor kinase \\
\hline GTP & Guanosine triphosphate \\
\hline GV & Gal4/VP16 transcription factor \\
\hline HA & Human influenza hemagglutinin \\
\hline His & Histamine \\
\hline HS & Horse serum \\
\hline $\mathrm{IC}_{50}$ & Half maximal inhibitory concentration \\
\hline $\mathrm{IP}_{3}$ & Inositol-1,4,5-trisphosphate \\
\hline Kan & Kanamycin \\
\hline LB & Luria Bertani \\
\hline MAPK & Mitogen-activated protein kinase \\
\hline MLP & Adenovirus major late promoter \\
\hline mRNA & Messenger RNA \\
\hline$n$ & Number \\
\hline $\mathrm{NaOH}$ & Sodium hydroxide \\
\hline NCBI & National Center for Biotechnology Information \\
\hline NFAT & Nuclear factor of activated T-cells \\
\hline NFKB & Nuclear factor-кB \\
\hline ON & Over night \\
\hline ORF & Open reading frame \\
\hline PAGE & Polyacrylamide gel electrophoresis \\
\hline PBS & Phosphate buffered saline \\
\hline PCR & Polymerase chain reaction \\
\hline
\end{tabular}




\begin{tabular}{ll} 
pENTR & Entry vector after BP recombination \\
pEXPR & Expressions vector after LR recombination \\
PGM & Personal Genome Machine \\
PI3K & Phosphoinositid-3-kinase \\
PIP 2 & Phosphatidylinositol-4,5-bisphosphate \\
PKA & Proteinkinase A \\
PKC & Proteinkinase C \\
PLL & Poly-L-Lysine \\
PMA & Phorbol 12-myristate 13-acetate \\
PNS & Peripheral nervous system \\
RLU & Relative luciferase units \\
RNA & Ribonucleic acid \\
RNase & Ribonuclease \\
RT & Room temperature \\
SD & Standard deviation \\
SEM & Standard error of the mean \\
SST & Somatostatin \\
SV40 & Simian virus 40 \\
TAE & Tris/acetate/EDTA \\
Taq & Thermus aquaticus \\
TE & Tris/EDTA \\
tet & Tetracycline \\
TEV & Tobacco etch virus \\
TK & Thymidine kinase \\
TRIS & tris(hydroxymethyl)aminomethane \\
UAS & Upstream activating sequence \\
UV & Ultraviolet \\
Zeo & Reoin \\
\hline
\end{tabular}




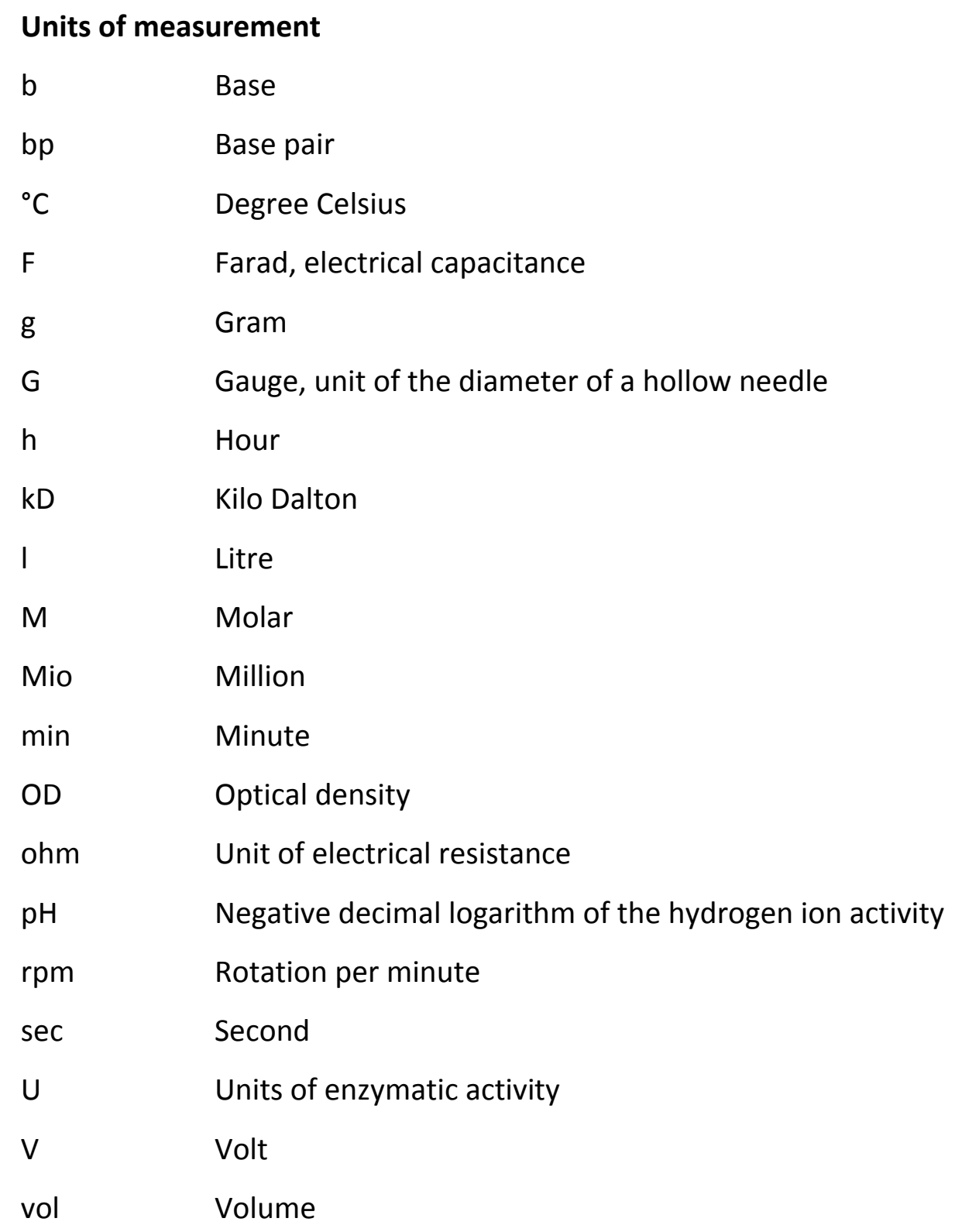

\section{Power prefixes}

$\begin{array}{ll}G & \text { Giga }\left(10^{9}\right) \\ M & \text { Mega }\left(10^{6}\right) \\ k & \text { Kilo }\left(10^{3}\right) \\ c & \text { Centi }\left(10^{-1}\right) \\ \mathrm{m} & \text { Milli }\left(10^{-3}\right) \\ \mu & \text { Micro }\left(10^{-6}\right) \\ \mathrm{n} & \text { Nano }\left(10^{-9}\right) \\ \mathrm{p} & \text { Pico }\left(10^{-12}\right) \\ \mathrm{f} & \text { Femto }\left(10^{-15}\right)\end{array}$




\section{DNA/RNA bases}
A
Adenine
C Cytidine
G Guanine
T Thymine
U Uracil

GPCRs

\begin{tabular}{|c|c|}
\hline HTR1A & Serotonin receptor $1 \mathrm{~A}$ \\
\hline HTR2A & Serotonin receptor $2 \mathrm{~A}$ \\
\hline HTR4 & Serotonin receptor 4 \\
\hline HTR5A & Serotonin receptor $5 \mathrm{~A}$ \\
\hline HTR6 & Serotonin receptor 6 \\
\hline HTR7 & Serotonin receptor 7 \\
\hline DRD1 & Dopamine receptor 1 \\
\hline DRD2 & Dopamine receptor 2 \\
\hline DRD3 & Dopamine receptor 3 \\
\hline DRD4 & Dopamine receptor 4 \\
\hline DRD5 & Dopamine receptor 5 \\
\hline ADRA1A & $\alpha$-adrenergic receptor $1 \mathrm{~A}$ \\
\hline ADRA2B & $\alpha$-adrenergic receptor $2 \mathrm{~B}$ \\
\hline ADRA2C & $\alpha$-adrenergic receptor $2 C$ \\
\hline ADRB2 & $\beta$-adrenergic receptor 2 \\
\hline ADRB3 & $\beta$-adrenergic receptor 3 \\
\hline $\mathrm{HRH} 1$ & Histamine receptor 1 \\
\hline AVPR1A & Arginine vasopressin receptor $1 \mathrm{~A}$ \\
\hline AVPR2 & Arginine vasopressin receptor 2 \\
\hline SSTR1 & Somatostatin receptor 1 \\
\hline SSTR2 & Somatostatin receptor 2 \\
\hline SSTR3 & Somatostatin receptor 3 \\
\hline
\end{tabular}




\section{Bibliography}

Allen, J.A., and Roth, B.L. (2011). Strategies to Discover Unexpected Targets for Drugs Active at $G$ Protein-Coupled Receptors. Annual Review of Pharmacology and Toxicology 51, 117-144.

Allen, J.A., Yost, J.M., Setola, V., Chen, X., Sassano, M.F., Chen, M., Peterson, S., Yadav, P.N., Huang, X., Feng, B., et al. (2011). Discovery of $\beta$-Arrestin-Biased Dopamine D2 Ligands for Probing Signal Transduction Pathways Essential for Antipsychotic Efficacy. Proceedings of the National Academy of Sciences.

Ayd (1961). A survey of drug-induced extrapyramidal reactions. JAMA 175, 1054-1060.

Baker, J.G. (2005). The selectivity of $\beta$-adrenoceptor antagonists at the human $\beta 1, \beta 2$ and $\beta 3$ adrenoceptors. Br J Pharmacol 144, 317-322.

Barnea, G., Strapps, W., Herrada, G., Berman, Y., Ong, J., Kloss, B., Axel, R., and Lee, K.J. (2008). The genetic design of signaling cascades to record receptor activation. Proc. Natl. Acad. Sci. U.S.A 105, 64-69.

Berg, K.A., Maayani, S., Goldfarb, J., Scaramellini, C., Leff, P., and Clarke, W.P. (1998). Effector Pathway-Dependent Relative Efficacy at Serotonin Type 2A and 2C Receptors: Evidence for Agonist-Directed Trafficking of Receptor Stimulus. Mol Pharmacol 54, 94104.

Bertrand, L., Parent, S., Caron, M., Legault, M., Joly, E., Angers, S., Bouvier, M., Brown, M., Houle, B., and Ménard, L. (2002). The BRET2/arrestin assay in stable recombinant cells: a platform to screen for compounds that interact with $G$ protein-coupled receptors (GPCRS). J. Recept. Signal Transduct. Res. 22, 533-541.

Bezzerri, V., Borgatti, M., Finotti, A., Tamanini, A., Gambari, R., and Cabrini, G. (2011). Mapping the Transcriptional Machinery of the IL-8 Gene in Human Bronchial Epithelial Cells. J Immunol 187, 6069-6081.

Botvinnik, A., and Rossner, M.J. (2012). Integrated Measurement of Split TEV and CisRegulatory Assays Using EXT Encoded Reporter Libraries. In Two Hybrid Technologies, B. Suter, and E.E. Wanker, eds. (Humana Press), pp. 309-323.

Botvinnik, A., Wichert, S.P., Fischer, T.M., and Rossner, M.J. (2010). Integrated analysis of receptor activation and downstream signaling with EXTassays. Nat Meth 7, 74-80.

Bowes, J., Brown, A.J., Hamon, J., Jarolimek, W., Sridhar, A., Waldron, G., and Whitebread, S. (2012). Reducing safety-related drug attrition: the use of in vitro pharmacological profiling. Nat Rev Drug Discov 11, 909-922.

Catapano, L.A., and Manji, H.K. (2007). G protein-coupled receptors in major psychiatric disorders. Biochimica et Biophysica Acta (BBA) - Biomembranes 1768, 976993. 
Chang, S.D., and Bruchas, M.R. (2014). Functional Selectivity at GPCRs: New Opportunities in Psychiatric Drug Discovery. Neuropsychopharmacology 39, 248-249.

Cheng, Z., Garvin, D., Paguio, A., Stecha, P., Wood, K., and Fan, F. (2010). Luciferase Reporter Assay System for Deciphering GPCR Pathways. Current Chemical Genomics 4, 84.

Conn, P.J., Tamminga, C., Schoepp, D.D., and Lindsley, C. (2008). Schizophrenia. Molecular Interventions 8, 99.

Corena-McLeod, M. (2015). Comparative Pharmacology of Risperidone and Paliperidone. Drugs in R\&D 15, 163.

Davis, K.L., Kahn, R.S., Ko, G., and Davidson, M. (1991). Dopamine in schizophrenia: a review and reconceptualization. Am J Psychiatry 148, 1474-1486.

DeWire, S.M., Ahn, S., Lefkowitz, R.J., and Shenoy, S.K. (2007). $\beta$-Arrestins and Cell Signaling. Annual Review of Physiology 69, 483-510.

Djannatian, M.S., Galinski, S., Fischer, T.M., and Rossner, M.J. (2011). Studying G protein-coupled receptor activation using split-tobacco etch virus assays. Analytical Biochemistry 412, 141-152.

Eidne, K.A., Kroeger, K.M., and Hanyaloglu, A.C. (2002). Applications of novel resonance energy transfer techniques to study dynamic hormone receptor interactions in living cells. Trends Endocrinol. Metab. 13, 415-421.

Emkey, R., and Rankl, N.B. (2009). Screening G Protein-Coupled Receptors: Measurement of Intracellular Calcium Using the Fluorometric Imaging Plate Reader. In High Throughput Screening, W.P. Janzen, and P. Bernasconi, eds. (Totowa, NJ: Humana Press), pp. 145-158.

Gilissen, J., Geubelle, P., Dupuis, N., Laschet, C., Pirotte, B., and Hanson, J. Forskolinfree cAMP assay for Gi-coupled receptors. Biochemical Pharmacology.

Greene, L.A., and Tischler, A.S. (1976). Establishment of a noradrenergic clonal line of rat adrenal pheochromocytoma cells which respond to nerve growth factor. Proc. Natl. Acad. Sci. U.S.A 73, 2424-2428.

Grewal, S.S., Fass, D.M., Yao, H., Ellig, C.L., Goodman, R.H., and Stork, P.J.S. (2000). Calcium and CAMP Signals Differentially Regulate cAMP-responsive Element-binding Protein Function via a Rap1-Extracellular Signal-regulated Kinase Pathway. Journal of Biological Chemistry 275, 34433-34441.

Gründer, G., Hippius, H., and Carlsson, A. (2009). The "atypicality" of antipsychotics: a concept re-examined and re-defined. Nat Rev Drug Discov 8, 197-202.

Guan, X.M., Kobilka, T.S., and Kobilka, B.K. (1992). Enhancement of membrane insertion and function in a type IIlb membrane protein following introduction of a cleavable signal peptide. J. Biol. Chem. 267, 21995-21998. 
Harrison, C., and Traynor, J.R. (2003). The [35S]GTPyS binding assay: approaches and applications in pharmacology. Life Sciences 74, 489-508.

Hartley, J.L., Temple, G.F., and Brasch, M.A. (2000). DNA cloning using in vitro sitespecific recombination. Genome Res. 10, 1788-1795.

Hattori, and Ozawa (2015). High-throughput Live Cell Imaging and Analysis for Temporal Reaction of G Protein-coupled Receptor Based on Split Luciferase Fragment Complementation. Analytical Sciences 327.

Hazel, T.G., Nathans, D., and Lau, L.F. (1988). A gene inducible by serum growth factors encodes a member of the steroid and thyroid hormone receptor superfamily. Proc Natl Acad Sci U S A 85, 8444-8448.

Heng, B.C., Aubel, D., Fussenegger, M., and xxx (2013). An overview of the diverse roles of G-protein coupled receptors (GPCRs) in the pathophysiology of various human diseases. Biotechnology Advances 31, 1676-1694.

Hepler, J.R., and Gilman, A.G. (1992). G proteins. Trends in Biochemical Sciences 17, 383-387.

Hoffmann, E., Dittrich-Breiholz, O., Holtmann, H., and Kracht, M. (2002). Multiple control of interleukin-8 gene expression. J. Leukoc. Biol. 72, 847-855.

Inaoka, Y., Yazawa, T., Uesaka, M., Mizutani, T., Yamada, K., and Miyamoto, K. (2008). Regulation of NGFI-B/Nur77 gene expression in the rat ovary and in leydig tumor cells MA-10. Mol. Reprod. Dev. 75, 931-939.

Insel, T.R. (2010). Rethinking schizophrenia. Nature 468, 187-193.

Johnson, C.M., Hill, C.S., Chawla, S., Treisman, R., and Bading, H. (1997). Calcium Controls Gene Expression via Three Distinct Pathways That Can Function Independently of the Ras/Mitogen-Activated Protein Kinases (ERKs) Signaling Cascade. J. Neurosci. 17, 6189-6202.

Kapust, R.B., Tözsér, J., Fox, J.D., Anderson, D.E., Cherry, S., Copeland, T.D., and Waugh, D.S. (2001). Tobacco etch virus protease: mechanism of autolysis and rational design of stable mutants with wild-type catalytic proficiency. Protein Eng. 14, 9931000.

Kenakin, T. (2011). Functional Selectivity and Biased Receptor Signaling. J Pharmacol Exp Ther 336, 296-302.

Kenakin, T., and Christopoulos, A. (2013). Signalling bias in new drug discovery: detection, quantification and therapeutic impact. Nat Rev Drug Discov 12, 205-216.

Kohout, T.A., and Lefkowitz, R.J. (2003). Regulation of G Protein-Coupled Receptor Kinases and Arrestins During Receptor Desensitization. Mol Pharmacol 63, 9-18. 
Komatsu, H. (2015). Novel Therapeutic GPCRs for Psychiatric Disorders. International Journal of Molecular Sciences 16, 14109.

Kovoor, A., Celver, J., Abdryashitov, R.I., Chavkin, C., and Gurevich, V.V. (1999). Targeted Construction of Phosphorylation-independent $\beta$-Arrestin Mutants with Constitutive Activity in Cells. Journal of Biological Chemistry 274, 6831-6834.

Kroeze, W.K., Sassano, M.F., Huang, X.-P., Lansu, K., McCorvy, J.D., Giguère, P.M., Sciaky, N., and Roth, B.L. (2015). PRESTO-Tango as an open-source resource for interrogation of the druggable human GPCRome. Nature Structural \& Molecular Biology 22, 362-369.

Lagerstrom, M.C., and Schioth, H.B. (2008). Structural diversity of G protein-coupled receptors and significance for drug discovery. Nat Rev Drug Discov 7, 339-357.

Lally, J., and MacCabe, J.H. (2015). Antipsychotic medication in schizophrenia: a review. Br Med Bull 114, 169-179.

Lanau, F., Zenner, M.-T., Civelli, O., and Hartman, D.S. (1997). Epinephrine and Norepinephrine Act as Potent Agonists at the Recombinant Human Dopamine D4 Receptor. Journal of Neurochemistry 68, 804-812.

Lawler, C.P., Prioleau, C., Lewis, M.M., Mak, C., Jiang, D., Schetz, J.A., Gonzalez, A.M., Sibley, D.R., and Mailman, R.B. (1999). Interactions of the Novel Antipsychotic Aripiprazole (OPC-14597) with Dopamine and Serotonin Receptor Subtypes. Neuropsychopharmacology 20, 612-627.

Lefkowitz, R.J., and Shenoy, S.K. (2005). Transduction of Receptor Signals by $\{$ beta\}Arrestins. Science 308, 512-517.

Leucht, S., Corves, C., Arbter, D., Engel, R.R., Li, C., and Davis, J.M. (2009). Secondgeneration versus first-generation antipsychotic drugs for schizophrenia: a metaanalysis. Lancet 373, 31-41.

Lieberman, J.A., Stroup, T.S., McEvoy, J.P., Swartz, M.S., Rosenheck, R.A., Perkins, D.O., Keefe, R.S.E., Davis, S.M., Davis, C.E., Lebowitz, B.D., et al. (2005). Effectiveness of Antipsychotic Drugs in Patients with Chronic Schizophrenia. New England Journal of Medicine 353, 1209-1223.

Luttrell, L.M., Ferguson, S.S.G., Daaka, Y., Miller, W.E., Maudsley, S., Rocca, G.J.D., Lin, F.-T., Kawakatsu, H., Owada, K., Luttrell, D.K., et al. (1999). $\beta$-Arrestin-Dependent Formation of $\beta 2$ Adrenergic Receptor-Src Protein Kinase Complexes. Science 283, 655661.

Mauri, M.C., Paletta, S., Maffini, M., Colasanti, A., Dragogna, F., Pace, C.D., and Altamura, A.C. (2014). Clinical pharmacology of atypical antipsychotics: an update. EXCLI Journal 13, 1163.

Maxwell, M.A., and Muscat, G.E.O. (2006). The NR4A subgroup: immediate early response genes with pleiotropic physiological roles. Nucl Recept Signal 4, e002. 
McGrath, J., Saha, S., Chant, D., and Welham, J. (2008). Schizophrenia: A Concise Overview of Incidence, Prevalence, and Mortality. Epidemiol Rev 30,67-76.

Miyamoto, S., Miyake, N., Jarskog, L.F., Fleischhacker, W.W., and Lieberman, J.A. (2012). Pharmacological treatment of schizophrenia: a critical review of the pharmacology and clinical effects of current and future therapeutic agents. Mol Psychiatry 17, 1206-1227.

Miyano, K., Sudo, Y., Yokoyama, A., Hisaoka-Nakashima, K., Morioka, N., Takebayashi, M., Nakata, Y., Higami, Y., and Uezono, Y. (2014). History of the G Protein-Coupled Receptor (GPCR) Assays From Traditional to a State-of-the-Art Biosensor Assay. Journal of Pharmacological Sciences 126, 302-309.

Mottola, D.M., Kilts, J.D., Lewis, M.M., Connery, H.S., Walker, Q.D., Jones, S.R., Booth, R.G., Hyslop, D.K., Piercey, M., Wightman, R.M., et al. (2002). Functional Selectivity of Dopamine Receptor Agonists. I. Selective Activation of Postsynaptic Dopamine D2 Receptors Linked to Adenylate Cyclase. J Pharmacol Exp Ther 301, 1166-1178.

Neves, S.R., Ram, P.T., and lyengar, R. (2002). G Protein Pathways. Science 296, 16361639.

Nunn, C.M., Jeeves, M., Cliff, M.J., Urquhart, G.T., George, R.R., Chao, L.H., Tscuchia, Y., and Djordjevic, S. (2005). Crystal Structure of Tobacco Etch Virus Protease Shows the Protein C Terminus Bound within the Active Site. Journal of Molecular Biology 350, 145-155.

Oakley, R.H., Laporte, S.A., Holt, J.A., Caron, M.G., and Barak, L.S. (2000). Differential Affinities of Visual Arrestin, $\beta A$ rrestin1, and $\beta$ Arrestin2 for $G$ Protein-coupled Receptors Delineate Two Major Classes of Receptors. Journal of Biological Chemistry $275,17201-17210$.

Oakley, R.H., Hudson, C.C., Cruickshank, R.D., Meyers, D.M., Payne, R.E., Rhem, S.M., and Loomis, C.R. (2002). The cellular distribution of fluorescently labeled arrestins provides a robust, sensitive, and universal assay for screening $G$ protein-coupled receptors. Assay Drug Dev Technol 1, 21-30.

Oueslati, N., Hounsou, C., Belhocine, A., Rodriguez, T., Dupuis, E., Zwier, J.M., Trinquet, E., Pin, J.-P., and Durroux, T. (2015). Time-resolved FRET strategy to screen GPCR ligand library. Methods Mol. Biol. 1272, 23-36.

Palczewski, K., Kumasaka, T., Hori, T., Behnke, C.A., Motoshima, H., Fox, B.A., Le Trong, I., Teller, D.C., Okada, T., Stenkamp, R.E., et al. (2000). Crystal structure of rhodopsin: A $\mathrm{G}$ protein-coupled receptor. Science $289,739-745$.

Papoian, T., Chiu, H.-J., Elayan, I., Jagadeesh, G., Khan, I., Laniyonu, A.A., Li, C.X., Saulnier, M., Simpson, N., and Yang, B. (2015). Secondary pharmacology data to assess potential off-target activity of new drugs: a regulatory perspective. Nat Rev Drug Discov 14, 294-294. 
Pitcher, J.A., Payne, E.S., Csortos, C., DePaoli-Roach, A.A., and Lefkowitz, R.J. (1995). The G-protein-coupled receptor phosphatase: a protein phosphatase type $2 \mathrm{~A}$ with a distinct subcellular distribution and substrate specificity. PNAS 92, 8343-8347.

Pontén, J., and Saksela, E. (1967). Two established in vitro cell lines from human mesenchymal tumours. Int. J. Cancer 2, 434-447.

Puck, T.T., Cieciura, S.J., and Robinson, A. (1958). Genetics of Somatic Mammalian Cells lii. Long-Term Cultivation of Euploid Cells from Human and Animal Subjects. J Exp Med 108, 945-956.

Rajagopal, S. (2013). Quantifying biased agonism: understanding the links between affinity and efficacy. Nat Rev Drug Discov 12, 483-483.

Rajagopal, S., Rajagopal, K., Lefkowitz, R.J., and xxx (2010). Teaching old receptors new tricks: biasing seven-transmembrane receptors. Nat Rev Drug Discov 9, 373-386.

Rey, E., Hernández-Díaz, F.J., Abreu, P., Alonso, R., and Tabares, L. (2001). Dopamine induces intracellular $\mathrm{Ca} 2+$ signals mediated by $\alpha 1 \mathrm{~B}$-adrenoceptors in rat pineal cells. European Journal of Pharmacology 430, 9-17.

Romanov, S., Medvedev, A., Gambarian, M., Poltoratskaya, N., Moeser, M., Medvedeva, L., Gambarian, M., Diatchenko, L., and Makarov, S. (2008). Homogeneous reporter system enables quantitative functional assessment of multiple transcription factors. Nat Meth 5, 253-260.

Roth, B.L., Sheffler, D.J., and Kroeze, W.K. (2004). Magic shotguns versus magic bullets: selectively non-selective drugs for mood disorders and schizophrenia. Nat Rev Drug Discov 3, 353-359.

Rothberg, J.M., Hinz, W., Rearick, T.M., Schultz, J., Mileski, W., Davey, M., Leamon, J.H., Johnson, K., Milgrew, M.J., Edwards, M., et al. (2011). An integrated semiconductor device enabling non-optical genome sequencing. Nature 475, 348-352.

Sadowski, I., Ma, J., Triezenberg, S., and Ptashne, M. (1988). GAL4-VP16 is an unusually potent transcriptional activator. Nature $335,563-564$.

Sanger, F., Nicklen, S., and Coulson, A.R. (1977). DNA sequencing with chainterminating inhibitors. Proc. Natl. Acad. Sci. U.S.A. 74, 5463-5467.

Shapiro, D.A., Renock, S., Arrington, E., Chiodo, L.A., Liu, L.-X., Sibley, D.R., Roth, B.L., and Mailman, R. (2003). Aripiprazole, A Novel Atypical Antipsychotic Drug with a Unique and Robust Pharmacology. Neuropsychopharmacology 28.

Shenoy, S.K., and Lefkowitz, R.J. (2003). Trafficking Patterns of $\beta$-Arrestin and G Protein-coupled Receptors Determined by the Kinetics of $\beta$-Arrestin Deubiquitination. J. Biol. Chem. 278, 14498-14506.

Siehler, S. (2007). G12/13-dependent signaling of G-protein-coupled receptors: disease context and impact on drug discovery. Expert Opin. Drug Discov. 2, 1591-1604. 
Southan, C., Sharman, J.L., Benson, H.E., Faccenda, E., Pawson, A.J., Alexander, S.P.H., Buneman, O.P., Davenport, A.P., McGrath, J.C., Peters, J.A., et al. (2015). The IUPHAR/BPS Guide to PHARMACOLOGY in 2016: towards curated quantitative interactions between 1300 protein targets and 6000 ligands. Nucleic Acids Res.

Stephenson, R.P. (1956). A modification of receptor theory. British Journal of Pharmacology and Chemotherapy 11, 379.

Stevens, R.C., Cherezov, V., Katritch, V., Abagyan, R., Kuhn, P., Rosen, H., and Wüthrich, K. (2013). The GPCR Network: a large-scale collaboration to determine human GPCR structure and function. Nat Rev Drug Discov 12, 25-34.

Tandon, R., Henry A. Nasrallah, and Matcheri S. Keshavan (2009). Schizophrenia, "just the facts" 4. Clinical features and conceptualization. Schizophr. Res. 110, 1-23.

Thomsen, W., Frazer, J., and Unett, D. (2005). Functional assays for screening GPCR targets. Current Opinion in Biotechnology 16, 655-665.

Ücok, A., and Gaebel, W. (2008). Side effects of atypical antipsychotics: a brief overview. World Psychiatry 7, 58-62.

Urban, J.D., Clarke, W.P., Zastrow, M. von, Nichols, D.E., Kobilka, B., Weinstein, H., Javitch, J.A., Roth, B.L., Christopoulos, A., Sexton, P.M., et al. (2007). Functional Selectivity and Classical Concepts of Quantitative Pharmacology. J Pharmacol Exp Ther 320, 1-13.

Wehr, M.C., and Rossner, M.J. Split protein biosensor assays in molecular pharmacological studies. Drug Discovery Today.

Wehr, M.C., Laage, R., Bolz, U., Fischer, T.M., Grunewald, S., Scheek, S., Bach, A., Nave, K.-A., and Rossner, M.J. (2006). Monitoring regulated protein-protein interactions using split TEV. Nat Meth 3, 985-993.

Wehr, M.C., Galinski, S., and Rossner, M.J. (2015). Monitoring G protein-coupled receptor activation using the protein fragment complementation technique split TEV. Methods Mol. Biol. 1272, 107-118.

Wettschureck, N., and Offermanns, S. (2005). Mammalian G Proteins and Their Cell Type Specific Functions. Physiological Reviews 85, 1159-1204.

Whalen, E.J., Rajagopal, S., and Lefkowitz, R.J. (2011). Therapeutic potential of $\beta$ arrestin- and G protein-biased agonists. Trends in Molecular Medicine 17, 126.

Williams, C. (2004). cAMP detection methods in HTS: selecting the best from the rest. Nat Rev Drug Discov 3, 125-135.

Xie, G.-X., and Palmer, P.P. (2007). How regulators of G protein signaling achieve selective regulation. J. Mol. Biol. 366, 349-365. 
Yan, Y.-X., Boldt-Houle, D.M., Tillotson, B.P., Gee, M.A., D’Eon, B.J., Chang, X.-J., Olesen, C.E.M., and Palmer, M.A.J. (2002). Cell-based high-throughput screening assay system for monitoring $\mathrm{G}$ protein-coupled receptor activation using beta-galactosidase enzyme complementation technology. J Biomol Screen 7, 451-459.

Zhang, and Xie, X. (2012). Tools for GPCR drug discovery. Acta Pharmacologica Sin 33, 372-384.

Zhang, J., Ferguson, S.S.G., Barak, L.S., Ménard, L., and Caron, M.G. (1996). Dynamin and $\beta$-Arrestin Reveal Distinct Mechanisms for $G$ Protein-coupled Receptor Internalization. J. Biol. Chem. 271, 18302-18305.

Zhang, W.-P., Ouyang, M., and Thomas, S.A. (2004). Potency of catecholamines and other L-tyrosine derivatives at the cloned mouse adrenergic receptors. Neuropharmacology 47, 438-449. 


\section{Acknowledgements}

First, I want to sincerely thank my supervisor Prof. Dr. Moritz Rossner for his support and all the knowledge and experience I gained from him. I am deeply grateful for always being available and open for discussions, ideas and good advice during all the time of this project.

I would like to extend my thanks to Dr. Sven Wichert for all the sequencing analysis work that he has done for this project and for his help and patience with all computerrelated problems and emergencies.

Thanks to Dr. Michael Wehr for advice, discussions and support. Special thanks for proof-reading my thesis.

I would like to thank Karin Neumeier for great support with RNA preparation and next generation sequencing; Nirmal Kannaiyan for his support in generating heatmaps; Dr. Ben Brankatschk for cloning the major part of the cis-sensor library; Dr. Wilko Hinrichs, Dr. Anna Botvinnik, Dr. Ali Shahmoradi, Alexandra Rupp, Katrin Stadler, Alexander Herholt, Dr. Dorota Badowska, Dr. Lisa Reinecke and Dr. Magdalena Brzózka for discussions and support in the lab.

I am grateful to my committee members, Prof. Dr. Martin Göpfert, Prof. Dr. Ernst Wimmer, Prof. Dr. Ralf Heinrich, Prof. Dr. Michael Sereda and Dr. Katrin Willig for taken part in my final exams.

A lot of thanks to my colleagues and friends at the Max Planck Institute of Experimental Medicine in Göttingen and at Ludwig Maximilian University in Munich for all their help, support, the good teamwork and friendship. 